\title{
On the Performances of Classical VAR and Sims-Zha Bayesian VAR Models in the Presence of Collinearity and Autocorrelated Error Terms
}

\author{
M. O. Adenomon*, V. A. Michael, O. P. Evans \\ Department of Mathematics \& Statistics, The Federal Polytechnic, Bida, Nigeria \\ Email: "admonsagie@gmail.com,vicronz@yahoo.com, patevansjj@gmail.com
}

Received 3 September 2015; accepted 22 February 2016; published 25 February 2016

Copyright (C) 2016 by authors and Scientific Research Publishing Inc.

This work is licensed under the Creative Commons Attribution International License (CC BY).

http://creativecommons.org/licenses/by/4.0/

(c) (i) Open Access

\begin{abstract}
In time series literature, many authors have found out that multicollinearity and autocorrelation usually afflict time series data. In this paper, we compare the performances of classical VAR and Sims-Zha Bayesian VAR models with quadratic decay on bivariate time series data jointly influenced by collinearity and autocorrelation. We simulate bivariate time series data for different collinearity levels $(-0.99,-0.95,-0.9,-0.85,-0.8,0.8,0.85,0.9,0.95,0.99)$ and autocorrelation levels $(-0.99,-0.95,-0.9,-0.85,-0.8,0.8,0.85,0.9,0.95,0.99)$ for time series length of $8,16,32,64,128$, 256 respectively. The results from 10,000 simulations reveal that the models performance varies with the collinearity and autocorrelation levels, and with the time series lengths. In addition, the results reveal that the BVAR4 model is a viable model for forecasting. Therefore, we recommend that the levels of collinearity and autocorrelation, and the time series length should be considered in using an appropriate model for forecasting.
\end{abstract}

\section{Keywords}

Vector Autoregression (VAR), Classical VAR, Bayesian VAR (BVAR), Sims-Zha Prior, Collinearity, Autocorrelation

\section{Introduction}

There are various objectives for studying time series. These include the understanding and description of the generated mechanism, the forecasting of future value and optimum control of a system [1]. In time series litera-

\footnotetext{
${ }^{*}$ Corresponding author.
}

How to cite this paper: Adenomon, M.O., Michael, V.A. and Evans, O.P. (2016) On the Performances of Classical VAR and Sims-Zha Bayesian VAR Models in the Presence of Collinearity and Autocorrelated Error Terms. Open Journal of Statistics, 6, 96-132. http://dx.doi.org/10.4236/ojs.2016.61012 
ture, many authors have found out that multicollinearity and autocorrelation usually afflict time series data. For instance, Gujarati [2] observed that multicollinearity problem usually afflicted the VAR models. It was reported that correlation coefficients $|r|>0.7$ was an appropriate indicator when collinearity began to severely distort model estimation and subsequent prediction [3]. In a recent work of Garba et al. [4], they observed that the autocorrelation problem usually afflicted time series data. Lastly, Adenomon and Oyejola [5] studied the performances of VAR and BVAR model (assuming harmonic decay) when the bivariate time series were jointly influenced by collinearity and autocorrelation.

The aim of this study is to examine the performances of the classical VAR and Sims-Zha Bayesian VAR model in the presence of collinearity and autocorrelated error terms.

\section{Model Description}

\subsection{Vector Autoregression (VAR) Model}

Given a set of $k$ time series variables, $y_{t}=\left[y_{i t}, \cdots, y_{K t}\right]^{\prime}$, VAR models of the form

$$
y_{t}=A_{1} y_{t-1}+\cdots+A_{p} y_{t-p}+u_{t}
$$

provide a fairly general framework for the Data General Process (DGP) of the series. More precisely this model is called a VAR process of order p or VAR(p) process. Here $u_{t}=\left[u_{1 t}, \cdots, u_{K t}\right]^{\prime}$ is a zero mean independent white noise process with non singular time invariant covariance matrix $\sum_{u}$ and the $A_{i}$ are $(k \times k)$ coefficient matrices. The process is easy to use for forecasting purpose though it is not easy to determine the exact relations between the variables represented by the VAR model in Equation (1) above [6].

Also, polynomial trends or seasonal dummies can be included in the model.

The process is stable if

$$
\operatorname{det}\left(I_{K}-A_{1} Z-\cdots-A_{p} z^{p}\right) \neq 0 \text { for }|z| \leq 1
$$

In that case it generates stationary time series with time invariant means and variance covariance structure. The basic assumptions and properties of a VAR processes is the stability condition. A VAR(p) processes is said to be stable or fulfills stability condition, if all its eigenvalues have modulus less than 1 [7].

Therefore To estimate the VAR model, one can write a VAR(p) with a concise matrix notation as:

$$
\begin{aligned}
& Y=B Z+U \\
& \text { where } Y=\left[y_{1}, \cdots, y_{T}\right], \quad Z_{t-1}=\left[\begin{array}{c}
y_{t-1} \\
\vdots \\
y_{t-p}
\end{array}\right], Z=\left[Z_{0}, \cdots, Z_{T-1}\right]
\end{aligned}
$$

Then the Multivariate Least Squares (MLS) for $B$ yields

$$
\hat{B}=\left(Z Z^{\prime}\right)^{-1} Z^{\prime} Y
$$

\subsection{Bayesian Vector Autoregression with Sims-Zha Prior}

In recent times, the BVAR model of Sims and Zha [8] has gained popularity both in economic time series and political analysis. The Sims-Zha BVAR allows for a more general specification and can produce a tractable multivariate normal posterior distribution. Again, the Sims-Zha BVAR estimates the parameters for the full system in a multivariate regression [9].

Given the reduced form model

$$
\begin{aligned}
& y_{t}=c+y_{t-1} B_{1}+\cdots+y_{t-p} B_{p}+u_{t} \\
& \text { where } c=d A_{0}^{-1}, B_{l}=-A_{1} A_{0}^{-1}, l=1,2, \cdots, p, u_{t}=\varepsilon_{t} A_{0}^{-1} \text { and } \Sigma=A_{0}^{-1^{\prime}} A_{0}^{-1}
\end{aligned}
$$

The matrix representation of the reduced form is given as:

$$
\underset{T \times m}{Y}=\underset{T \times(m p+1)(m p+1) \times m}{X} \underset{T \times m}{\beta}, \quad U \sim \operatorname{MVN}(0, \Sigma)
$$

We can then construct a reduced form Bayesian SUR with the Sims-Zha prior as follows. The prior means for 
the reduced form coefficients are that $B_{1}=I$ and $B_{2}, \cdots, B_{p}=0$. We assume that the prior has a conditional structure that is multivariate Normal-inverse Wishart distribution for the parameters in the model. To estimate the coefficients for the system of the reduced form model with the following estimators

$$
\begin{aligned}
& \hat{\beta}=\left(\Psi^{-1}+X^{\prime} X\right)^{-1}\left(\Psi^{-1} \bar{\beta}+X^{\prime} Y\right) \\
& \hat{\Sigma}=T^{-1}\left(Y^{\prime} Y-\hat{\beta}^{\prime}\left(X^{\prime} X+\Psi^{-1}\right) \hat{\beta}+\bar{\beta}^{\prime} \Psi^{-1} \bar{\beta}+\bar{S}\right)
\end{aligned}
$$

where the Normal-inverse Wishart prior for the coefficients is

$$
\beta / \Sigma \sim N(\bar{\beta}, \Psi) \text { and } \Sigma \sim I W(\bar{S}, v)
$$

This representation translates the prior proposed by Sims and Zha form from the structural model to the reduced form ([10] [9]), and ([8] [11]).

The summary of the Sims-Zha prior is given in Table 1.

\section{Simulation Procedure}

The simulation procedure is as follows:

Step 1: We generated an artificial two-dimensional (Bivariate data) VAR (2) process that obeys the following form:

$$
\left[\begin{array}{l}
y_{1} \\
y_{2}
\end{array}\right]_{t}=\left[\begin{array}{c}
5.0 \\
10.0
\end{array}\right]+\left[\begin{array}{cc}
0.5 & 0.2 \\
-0.2 & -0.5
\end{array}\right]\left[\begin{array}{l}
y_{1} \\
y_{2}
\end{array}\right]_{t-1}+\left[\begin{array}{cc}
-0.3 & -0.7 \\
-0.1 & 0.3
\end{array}\right]\left[\begin{array}{l}
y_{1} \\
y_{2}
\end{array}\right]_{t-2}+\left[\begin{array}{l}
u_{1} \\
u_{2}
\end{array}\right]_{t}
$$

such that $u_{1 t}=\delta u_{1 t}+\varepsilon_{1 t}$ and $u_{2 t}=\delta u_{2 t-1}+\varepsilon_{2 t}$ where $\varepsilon_{i t} \sim N(0,1)$ and $i=1,2, \cdots, T$. Our choice here is similar to the work and illustration of Cowpertwait, [12]. We considered ten autocorrelated levels as $\delta=(-0.99,-0.95,-0.9,-0.85,-0.8,0.8,0.85,0.9,0.95,0.99)$. Our choice for this form model is to obtain a stable process and a VAR process with a true lag length [13].

Step 2: We then use the Cholesky Decomposition to apply to the data generated in Step $\mathbf{1}$ in order to create a bivariate time series data so that $y_{1}$ and $y_{2}$ have the desired correlation level [14]. We considered ten multicollinearity levels as $\rho=(-0.99,-0.95,-0.9,-0.85,-0.8,0.8,0.85,0.9,0.95,0.99)$.

The combination of Step 1 and 2 therefore produce a bivariate time series such that $y_{1}$ and $y_{2}$ are jointly influenced by multicollinearity and autocorrelation.

The simulated data assumed time series lengths of 8, 16, 32, 64, 128 and 256. A sample of simulated data is presented in Table 2 below.

\subsection{Model Specification}

The time series were generated data using a VAR model with lag 2. The choice here is to obtain a bivariate time series with the true lag length. While the VAR and BVAR models of lag length of 2 was used for modeling and forecasting purpose.

Table 1. Hyperparameters of Sims-Zha reference prior.

\begin{tabular}{ccl}
\hline Parameter & Range & \multicolumn{1}{c}{ Interpretation } \\
\hline$\lambda_{0}$ & {$[0,1]$} & Overall scale of the error covariance matrix \\
$\lambda_{1}$ & $>0$ & Standard deviation around $\mathrm{A}_{1}$ (persistence) \\
$\lambda_{2}$ & $=1$ & Weight of own lag versus other lags \\
$\lambda_{3}$ & $>0$ & Lag decay \\
$\lambda_{4}$ & $\geq 0$ & Scale of standard deviation of intercept \\
$\lambda_{5}$ & $\geq 0$ & Scale of standard deviation of exogenous variable coefficients \\
$\mu_{5}$ & $\geq 0$ & Sum of coefficients/Cointegration (long-term trends) \\
$\mu_{6}$ & $\geq 0$ & Initial observations/dummy observation (impacts of initial conditions) \\
$v$ & $>0$ & Prior degrees of freedom \\
\hline
\end{tabular}

Source: Brandt and Freeman, [10]. 
Table 2. Sample of simulated data for $T=8$.

\begin{tabular}{rcccccc}
\hline & \multicolumn{2}{r}{$\rho-9$} & \multicolumn{2}{c}{ Residuals } & \multicolumn{2}{c}{$\delta=0.9$} \\
\hline & $y_{1}$ & $y_{2}$ & {$[\mathrm{U}, 1]$} & {$[\mathrm{U}, 2]$} & -0.36417791 & -1.05632564 \\
\hline 1 & 4.635822 & 8.562592 & 0.55065249 & 0.3621112 & -2.63216817 & -2.31257463 \\
$\mathbf{2}$ & 8.276017 & 9.102328 & 0.90145401 & 0.6982855 & -0.97256527 & -1.03760062 \\
$\mathbf{3}$ & -1.176551 & 2.678471 & 1.46132912 & 1.0779790 & 0.19179026 & 0.85192775 \\
$\mathbf{4}$ & -1.324869 & 1.132437 & -8.63500727 & -5.8296621 & 0.07215923 & -0.24242029 \\
$\mathbf{5}$ & -1.217209 & 2.879577 & -0.02956038 & -1.4306597 & -0.43158786 & -0.92545664 \\
$\mathbf{6}$ & 1.841691 & 4.163813 & 0.11858453 & 1.7689057 & -1.16868494 & -1.66643992 \\
$\mathbf{7}$ & 1.244165 & 4.437567 & 3.15291385 & 1.3667331 & -2.00270147 & -2.87661209 \\
$\mathbf{8}$ & 3.418945 & 5.086300 & -0.30830834 & 0.4905413 & & \\
& & & 2.45848960 & 0.8676871 & & Estimated value $=0.616$ \\
\hline
\end{tabular}

For the BVAR model with Sims-Zha prior, we considered the following range of values for the hyperparameters given below and the Normal-inverse Wishart prior was employed.

We considered two tight priors and two loose priors as follows:

The Tight priors are as follows

$$
\begin{aligned}
& \operatorname{BVAR} 1=\left(\lambda_{0}=0.6, \lambda_{1}=0.1, \lambda_{3}=2, \lambda_{4}=0.1, \lambda_{5}=0.07, \mu_{5}=\mu_{6}=5\right) \\
& \operatorname{BVAR} 2=\left(\lambda_{0}=0.8, \lambda_{1}=0.1, \lambda_{3}=2, \lambda_{4}=0.1, \lambda_{5}=0.07, \mu_{5}=\mu_{6}=5\right)
\end{aligned}
$$

The Loose priors are as follows

$$
\begin{aligned}
& \operatorname{BVAR} 3=\left(\lambda_{0}=0.6, \lambda_{1}=0.15, \lambda_{3}=2, \lambda_{4}=0.15, \lambda_{5}=0.07, \mu_{5}=\mu_{6}=2\right) \\
& \operatorname{BVAR} 4=\left(\lambda_{0}=0.8, \lambda_{1}=0.15, \lambda_{3}=2, \lambda_{4}=0.15, \lambda_{5}=0.07, \mu_{5}=\mu_{6}=2\right)
\end{aligned}
$$

where $n \mu$ is prior degrees of freedom given as $m+1$ where $m$ is the number of variables in the multiple time series data. In work $n \mu$ is 3 (that is two (2) time series variables plus 1 (one)).

Our choice of Normal-inverse Wishart prior for the BVAR models follow the work of Kadiyala \& Karlsson, [15] that Normal Wishart prior tends to performed better when compared to other priors. In addition Sims and Zha, [8] proposed Normal-inverse Wishart prior because of its suitability for large systems while Breheny, [16] reported that the most advantage of Wishart distribution is that it guaranteed to produce positive definite draws. Our choice of the overall tightness $\lambda_{0}=0.6$ and 0.8 is in line with work of Brandt, Colaresi and Freeman, [17]. In this work we assumed that the bivariate time series follows a quadratic decay. The Quadratic Decay (QD) model has many attractive theoretical properties that is why it is been applied to many fields of endeavour ([18] [19]) [20].

The following are the criteria for Forecast assessments used:

1) Mean Absolute Error (MAE) has a formular $\mathrm{MAE}_{j}=\frac{\sum_{i=1}^{n}\left|e_{i}\right|}{n}$. This criterion measures deviation from the series in absolute terms, and measures how much the forecast is biased. This measure is one of the most common ones used for analyzing the quality of different forecasts.

2) The Root Mean Square Error (RMSE) is given as $\operatorname{RMSE}_{j}=\sqrt{\frac{\sum_{i}^{n}\left(y_{i}-y^{f}\right)^{2}}{n}}$ where $y_{i}$ is the time series data and $y^{f}$ is the forecast value of $y$ [13].

For the two measures above, the smaller the value, the better the fit of the model [21].

In this simulation study, $\mathrm{RMSE}=\frac{\sum_{j}^{N} \mathrm{RMSE}_{j}}{N}$ and MAE $=\frac{\sum_{j}^{N} \mathrm{MAE}_{j}}{N}$ where $N=10,000$. Therefore, the 
model with the minimum RMSE and MAE result as the preferred model.

\subsection{Statistical Packages (R)}

In this study three procedures in the $R$ package will be used. They are: Dynamic System Estimation (DSE) [22]; the vars [23], and the MSBVAR [24].

\section{Results and Discussion}

The Root Mean Square Error (RMSE) and Mean Absolute Error (MAE) were obtained for the various models in the work and are presented in Appendix A, while the ranks are presented in Appendix B. But here the preferred models with respect to their rank are presented. The preferred model for the time series length of $8,16,32,64$, 128 and 256 are presented in Tables 3-8 respectively.

In Table 3 below, when $T=8$ the BVAR models are preferred in all levels of collinearity and autocorrelation. Also in Table 4 below, when $T=16$ the BVAR models are preferred in all levels of collinearity and autocorrelation.

In Table 5 below, when $T=32$ the BVAR models are preferred in all levels of collinearity and autocorrelation except in few cases where classical VAR is preferred. In Table 6 below, when $T=64$ the BVAR models are preferred in all levels of collinearity and autocorrelation except in some cases where classical VAR is preferred.

In Table 7 below, when $T=128$ the BVAR models are preferred in some levels of collinearity and autocorre-

Table 3. Preferred model at different levels of collinearity and autocorrelation when $T=8$.

\begin{tabular}{ccccccccccccc}
\hline \multicolumn{10}{c}{ AUTOCO } & \multicolumn{10}{c}{-0.99} & \multicolumn{10}{c}{ COLLINERITY $(\rho)$} \\
LEVELS $(\delta)$ & RMSE & MAE & RMSE & MAE & RMSE & MAE & RMSE & MAE & RMSE & MAE & RMSE & MAE \\
\hline-0.99 & BVAR4 & BVAR4 & BVAR4 & BVAR4 & BVAR4 & BVAR4 & BVAR4 & BVAR4 & BVAR4 & BVAR4 & BVAR4 & BVAR2 \\
-0.95 & BVAR4 & BVAR4 & BVAR4 & BVAR4 & BVAR4 & BVAR4 & BVAR4 & BVAR4 & BVAR4 & BVAR4 & BVAR3 & BVAR2 \\
-0.9 & BVAR4 & BVAR4 & BVAR4 & BVAR4 & BVAR4 & BVAR4 & BVAR4 & BVAR4 & BVAR4 & BVAR4 & BVAR2 & BVAR1 \\
-0.85 & BVAR4 & BVAR4 & BVAR4 & BVAR4 & BVAR4 & BVAR4 & BVAR4 & BVAR4 & BVAR4 & BVAR4 & BVAR1 & BVAR1 \\
-0.8 & BVAR3 & BVAR3 & BVAR4 & BVAR4 & BVAR4 & BVAR4 & BVAR4 & BVAR4 & BVAR4 & BVAR4 & BVAR1 & BVAR1 \\
0.8 & BVAR1 & BVAR1 & BVAR3 & BVAR3 & BVAR4 & BVAR4 & BVAR4 & BVAR4 & BVAR4 & BVAR3 & BVAR1 & BVAR1 \\
0.85 & BVAR3 & BVAR1 & BVAR3 & BVAR3 & BVAR4 & BVAR4 & BVAR4 & BVAR3 & BVAR3 & BVAR3 & BVAR1 & BVAR1 \\
0.9 & BVAR1 & BVAR1 & BVAR4 & BVAR4 & BVAR4 & BVAR4 & BVAR4 & BVAR4 & BVAR4 & BVAR3 & BVAR1 & BVAR1 \\
0.95 & BVAR3 & BVAR1 & BVAR3 & BVAR3 & BVAR4 & BVAR4 & BVAR4 & BVAR4 & BVAR4 & BVAR4 & BVAR1 & BVAR1 \\
0.99 & BVAR1 & BVAR1 & BVAR4 & BVAR3 & BVAR4 & BVAR3 & BVAR4 & BVAR3 & BVAR4 & BVAR4 & BVAR1 & BVAR1 \\
\hline
\end{tabular}

\begin{tabular}{cccccccccc}
\hline & \multicolumn{7}{c}{ COLLINEARITY $(\rho)$} \\
AUTOCO & \multicolumn{2}{c}{0.85} & \multicolumn{2}{c}{0.95} & & \multicolumn{2}{c}{0.99} \\
\cline { 2 - 8 }$(\delta)$ & RMSE & MAE & RMSE & MAE & RMSE & MAE & RMSE & MAE \\
\hline-0.99 & BVAR4 & BVAR1 & BVAR3 & BVAR1 & BVAR4 & BVAR1 & BVAR4 & BVAR1 \\
-0.95 & BVAR3 & BVAR1 & BVAR1 & BVAR1 & BVAR1 & BVAR1 & BVAR3 & BVAR1 \\
-0.9 & BVAR1 & BVAR1 & BVAR2 & BVAR1 & BVAR1 & BVAR1 & BVAR3 & BVAR1 \\
-0.85 & BVAR1 & BVAR1 & BVAR1 & BVAR1 & BVAR2 & BVAR1 & BVAR1 & BVAR1 \\
-0.8 & BVAR1 & BVAR1 & BVAR1 & BVAR1 & BVAR1 & BVAR1 & BVAR2 & BVAR1 \\
0.8 & BVAR1 & BVAR1 & BVAR1 & BVAR1 & BVAR1 & BVAR1 & BVAR1 & BVAR1 \\
0.85 & BVAR1 & BVAR1 & BVAR1 & BVAR1 & BVAR1 & BVAR1 & BVAR1 & BVAR1 \\
0.9 & BVAR1 & BVAR1 & BVAR2 & BVAR1 & BVAR2 & BVAR1 & BVAR1 & BVAR1 \\
0.95 & BVAR1 & BVAR1 & BVAR1 & BVAR1 & BVAR1 & BVAR1 & BVAR1 & BVAR1 \\
0.99 & BVAR2 & BVAR1 & BVAR1 & BVAR1 & BVAR1 & BVAR1 & BVAR1 & BVAR1 \\
\hline
\end{tabular}


lation and the classical VAR is preferred in some levels of collinearity and autocorrelation. In Table 8 below, when $T=256$ the BVAR models are preferred in some levels of collinearity and autocorrelation and the classical VAR is preferred in some levels of collinearity and autocorrelation.

Table 4. Preferred model at different levels of collinearity and autocorrelation when $T=16$.

\begin{tabular}{|c|c|c|c|c|c|c|c|c|c|c|c|c|}
\hline \multirow{3}{*}{$\begin{array}{c}\text { AUTOCO } \\
\text { LEVELS }(\delta)\end{array}$} & \multicolumn{12}{|c|}{ COLLINERITY $(\rho)$} \\
\hline & \multicolumn{2}{|c|}{-0.99} & \multicolumn{2}{|c|}{-0.95} & \multicolumn{2}{|c|}{-0.9} & \multicolumn{2}{|c|}{-0.85} & \multicolumn{2}{|c|}{-0.8} & \multicolumn{2}{|c|}{0.8} \\
\hline & RMSE & MAE & RMSE & MAE & RMSE & MAE & RMSE & MAE & RMSE & MAE & RMSE & MAE \\
\hline-0.99 & BVAR4 & BVAR4 & BVAR4 & BVAR4 & BVAR4 & BVAR4 & BVAR4 & BVAR4 & BVAR4 & BVAR4 & BVAR4 & BVAR4 \\
\hline-0.95 & BVAR4 & BVAR4 & BVAR4 & BVAR4 & BVAR4 & BVAR4 & BVAR4 & BVAR4 & BVAR4 & BVAR4 & BVAR3 & BVAR3 \\
\hline-0.9 & BVAR4 & BVAR4 & BVAR4 & BVAR4 & BVAR4 & BVAR4 & BVAR4 & BVAR4 & BVAR4 & BVAR4 & BVAR3 & BVAR2 \\
\hline-0.85 & BVAR4 & BVAR4 & BVAR4 & BVAR4 & BVAR4 & BVAR4 & BVAR4 & BVAR4 & BVAR4 & BVAR4 & BVAR2 & BVAR2 \\
\hline-0.8 & BVAR4 & BVAR4 & BVAR4 & BVAR4 & BVAR4 & BVAR4 & BVAR4 & BVAR4 & BVAR4 & BVAR4 & BVAR1 & BVAR1 \\
\hline 0.8 & BVAR4 & BVAR4 & BVAR4 & BVAR4 & BVAR4 & BVAR4 & BVAR4 & BVAR4 & BVAR4 & BVAR4 & BVAR1 & BVAR1 \\
\hline 0.85 & BVAR4 & BVAR4 & BVAR4 & BVAR4 & BVAR4 & BVAR4 & BVAR4 & BVAR4 & BVAR4 & BVAR4 & BVAR2 & BVAR2 \\
\hline 0.9 & BVAR4 & BVAR4 & BVAR4 & BVAR4 & BVAR4 & BVAR4 & BVAR4 & BVAR4 & BVAR4 & BVAR4 & BVAR1 & BVAR1 \\
\hline 0.95 & BVAR4 & BVAR4 & BVAR4 & BVAR4 & BVAR4 & BVAR4 & BVAR4 & BVAR4 & BVAR4 & BVAR4 & BVAR1 & BVAR1 \\
\hline 0.99 & BVAR3 & BVAR3 & BVAR4 & BVAR4 & BVAR4 & BVAR4 & BVAR4 & BVAR4 & BVAR4 & BVAR4 & BVAR2 & BVAR2 \\
\hline
\end{tabular}

\begin{tabular}{|c|c|c|c|c|c|c|c|c|}
\hline \multirow{3}{*}{$\begin{array}{c}\text { AUTOCO } \\
\text { LEVELS }(\delta)\end{array}$} & \multicolumn{8}{|c|}{ COLLINEARITY $(\rho)$} \\
\hline & \multicolumn{2}{|c|}{0.85} & \multicolumn{2}{|c|}{0.9} & \multicolumn{2}{|c|}{0.95} & \multicolumn{2}{|c|}{0.99} \\
\hline & RMSE & MAE & RMSE & MAE & RMSE & MAE & RMSE & MAE \\
\hline-0.99 & BVAR4 & BVAR4 & BVAR4 & BVAR4 & BVAR4 & BVAR4 & BVAR4 & BVAR4 \\
\hline-0.95 & BVAR3 & BVAR3 & BVAR4 & BVAR2 & BVAR4 & BVAR4 & BVAR4 & BVAR4 \\
\hline-0.9 & BVAR4 & BVAR2 & BVAR4 & BVAR2 & BVAR4 & BVAR2 & BVAR4 & BVAR4 \\
\hline-0.85 & BVAR2 & BVAR2 & BVAR2 & BVAR2 & BVAR4 & BVAR2 & BVAR4 & BVAR4 \\
\hline-0.8 & BVAR2 & BVAR1 & BVAR1 & BVAR1 & BVAR2 & BVAR1 & BVAR4 & BVAR4 \\
\hline 0.8 & BVAR1 & BVAR1 & BVAR1 & BVAR1 & BVAR1 & BVAR1 & BVAR1 & BVAR1 \\
\hline 0.85 & BVAR1 & BVAR1 & BVAR1 & BVAR1 & BVAR1 & BVAR1 & BVAR2 & BVAR2 \\
\hline 0.9 & BVAR1 & BVAR1 & BVAR1 & BVAR1 & BVAR1 & BVAR1 & BVAR4 & BVAR1 \\
\hline 0.95 & BVAR1 & BVAR1 & BVAR2 & BVAR2 & BVAR1 & BVAR1 & BVAR3 & BVAR1 \\
\hline 0.99 & BVAR1 & BVAR1 & BVAR1 & BVAR1 & BVAR1 & BVAR1 & BVAR3 & BVAR2 \\
\hline
\end{tabular}

Table 5. Preferred model at different levels of collinearity and autocorrelation when $T=32$.

\begin{tabular}{|c|c|c|c|c|c|c|c|c|c|c|c|c|}
\hline \multirow{3}{*}{$\begin{array}{c}\text { AUTOCO } \\
\text { LEVELS }(\delta)\end{array}$} & \multicolumn{12}{|c|}{ COLLINERITY $(\rho)$} \\
\hline & \multicolumn{2}{|c|}{-0.99} & \multicolumn{2}{|c|}{-0.95} & \multicolumn{2}{|c|}{-0.9} & \multicolumn{2}{|c|}{-0.85} & \multicolumn{2}{|c|}{-0.8} & \multicolumn{2}{|c|}{0.8} \\
\hline & RMSE & MAE & RMSE & MAE & RMSE & MAE & RMSE & MAE & RMSE & MAE & RMSE & MAE \\
\hline-0.99 & BVAR4 & BVAR4 & BVAR4 & BVAR4 & BVAR4 & BVAR4 & BVAR4 & BVAR4 & BVAR4 & BVAR4 & BVAR4 & BVAR4 \\
\hline-0.95 & BVAR4 & BVAR4 & BVAR4 & BVAR4 & BVAR3 & BVAR3 & BVAR4 & BVAR4 & BVAR4 & BVAR4 & BVAR2 & BVAR2 \\
\hline-0.9 & BVAR4 & BVAR4 & BVAR4 & BVAR4 & BVAR4 & BVAR4 & BVAR4 & BVAR4 & BVAR4 & BVAR4 & BVAR2 & BVAR2 \\
\hline-0.85 & BVAR4 & BVAR3 & BVAR4 & BVAR4 & BVAR4 & BVAR4 & BVAR3 & BVAR3 & BVAR4 & BVAR3 & BVAR2 & BVAR2 \\
\hline-0.8 & BVAR4 & BVAR4 & BVAR4 & BVAR4 & BVAR4 & BVAR4 & BVAR4 & BVAR4 & BVAR4 & BVAR4 & VAR(2) & $\operatorname{VAR}(2)$ \\
\hline 0.8 & BVAR4 & BVAR4 & BVAR4 & BVAR4 & BVAR4 & BVAR4 & BVAR4 & BVAR4 & BVAR4 & BVAR4 & VAR(2) & $\operatorname{VAR}(2)$ \\
\hline 0.85 & BVAR4 & BVAR4 & BVAR4 & BVAR4 & BVAR4 & BVAR4 & BVAR4 & BVAR4 & BVAR4 & BVAR4 & BVAR4 & VAR(2 \\
\hline 0.9 & BVAR4 & BVAR4 & BVAR4 & BVAR4 & BVAR4 & BVAR4 & BVAR4 & BVAR4 & BVAR4 & BVAR4 & BVAR4 & BVAR4 \\
\hline 0.95 & BVAR4 & BVAR4 & BVAR4 & BVAR4 & BVAR4 & BVAR4 & BVAR4 & BVAR4 & BVAR4 & BVAR4 & BVAR4 & BVAR4 \\
\hline 0.99 & BVAR3 & BVAR3 & BVAR4 & BVAR4 & BVAR4 & BVAR4 & BVAR3 & BVAR3 & BVAR3 & BVAR3 & BVAR4 & BVAR4 \\
\hline
\end{tabular}




\begin{tabular}{|c|c|c|c|c|c|c|c|c|}
\hline \multirow{3}{*}{$\begin{array}{c}\text { AUTOCO } \\
\text { LEVELS }(\delta)\end{array}$} & \multicolumn{8}{|c|}{ COLLINEARITY $(\rho)$} \\
\hline & \multicolumn{2}{|c|}{0.85} & \multicolumn{2}{|c|}{0.9} & \multicolumn{2}{|c|}{0.95} & \multicolumn{2}{|c|}{0.99} \\
\hline & RMSE & MAE & RMSE & MAE & RMSE & MAE & RMSE & MAE \\
\hline-0.99 & BVAR4 & BVAR4 & BVAR4 & BVAR2 & BVAR4 & BVAR4 & BVAR4 & BVAR4 \\
\hline-0.95 & BVAR2 & BVAR2 & BVAR2 & BVAR2 & BVAR2 & BVAR2 & BVAR4 & BVAR4 \\
\hline-0.9 & BVAR2 & BVAR2 & BVAR2 & BVAR2 & BVAR2 & BVAR2 & BVAR4 & BVAR4 \\
\hline-0.85 & BVAR2 & VAR(2) & BVAR2 & BVAR2 & BVAR2 & BVAR2 & BVAR4 & BVAR4 \\
\hline-0.8 & BVAR2 & VAR(2) & BVAR2 & VAR(2) & BVAR2 & VAR(2) & BVAR4 & BVAR4 \\
\hline 0.8 & VAR(2) & VAR(2) & VAR(2) & VAR(2) & VAR(2) & VAR(2) & BVAR4 & BVAR4 \\
\hline 0.85 & BVAR4 & VAR(2) & BVAR4 & BVAR4 & BVAR4 & BVAR4 & BVAR4 & BVAR4 \\
\hline 0.9 & BVAR4 & BVAR4 & BVAR4 & BVAR4 & BVAR4 & BVAR4 & BVAR4 & BVAR4 \\
\hline 0.95 & BVAR4 & BVAR4 & BVAR4 & BVAR4 & BVAR4 & BVAR4 & BVAR4 & BVAR4 \\
\hline 0.99 & BVAR4 & BVAR4 & BVAR3 & BVAR3 & BVAR4 & BVAR4 & BVAR4 & BVAR4 \\
\hline
\end{tabular}

Table 6. Preferred model at different levels of collinearity and autocorrelation when $T=64$.

\begin{tabular}{|c|c|c|c|c|c|c|c|c|c|c|c|c|}
\hline \multirow{3}{*}{$\begin{array}{c}\text { AUTOCO } \\
\text { LEVELS }(\delta)\end{array}$} & \multicolumn{12}{|c|}{ COLLINERITY $(\rho)$} \\
\hline & \multicolumn{2}{|c|}{-0.99} & \multicolumn{2}{|c|}{-0.95} & \multicolumn{2}{|c|}{-0.9} & \multicolumn{2}{|c|}{-0.85} & \multicolumn{2}{|c|}{-0.8} & \multicolumn{2}{|r|}{0.8} \\
\hline & RMSE & \multirow{2}{*}{$\begin{array}{c}\text { MAE } \\
\text { BVAR4 }\end{array}$} & \multirow{2}{*}{$\begin{array}{c}\text { RMSE } \\
\text { BVAR4 }\end{array}$} & MAE & \multirow{2}{*}{$\begin{array}{c}\text { RMSE } \\
\text { BVAR4 }\end{array}$} & \multirow{2}{*}{$\begin{array}{c}\text { MAE } \\
\text { BVAR4 }\end{array}$} & \multirow{2}{*}{$\begin{array}{c}\text { RMSE } \\
\text { BVAR4 }\end{array}$} & \multirow{2}{*}{$\begin{array}{c}\text { MAE } \\
\text { BVAR4 }\end{array}$} & \multirow{2}{*}{$\begin{array}{c}\text { RMSE } \\
\text { BVAR4 }\end{array}$} & \multirow{2}{*}{$\begin{array}{c}\text { MAE } \\
\text { BVAR4 }\end{array}$} & RMSE & MAE \\
\hline-0.99 & BVAR4 & & & BVAR4 & & & & & & & BVAR4 & 4 BVAR4 \\
\hline-0.95 & BVAR2 & BVAR2 & BVAR2 & BVAR2 & 2 BVAR4 & BVAR4 & BVAR4 & BVAR2 & BVAR4 & BVAR1 & BVAR2 & 2 BVAR2 \\
\hline-0.9 & BVAR2 & BVAR3 & BVAR2 & BVAR2 & 2 BVAR4 & BVAR2 & BVAR4 & BVAR1 & BVAR4 & BVAR4 & BVAR2 & $2 \operatorname{VAR}(2)$ \\
\hline-0.85 & BVAR2 & BVAR2 & BVAR1 & BVAR1 & 1 BVAR4 & BVAR4 & BVAR4 & BVAR4 & BVAR4 & BVAR4 & $\operatorname{VAR}(2)$ & ) $\operatorname{VAR}(2)$ \\
\hline-0.8 & BVAR2 & BVAR2 & BVAR4 & BVAR4 & 4 BVAR4 & BVAR4 & BVAR4 & BVAR4 & BVAR4 & BVAR4 & $\operatorname{VAR}(2)$ & VAR(2) \\
\hline 0.8 & BVAR4 & BVAR4 & BVAR4 & BVAR4 & 4 BVAR4 & BVAR4 & BVAR4 & BVAR4 & BVAR4 & BVAR4 & $\operatorname{VAR}(2)$ & $\operatorname{VAR}(2)$ \\
\hline 0.85 & BVAR4 & BVAR4 & BVAR4 & BVAR4 & 4 BVAR4 & BVAR4 & BVAR4 & BVAR4 & BVAR4 & BVAR4 & $\operatorname{VAR}(2)$ & VAR(2) \\
\hline 0.9 & BVAR4 & BVAR4 & BVAR4 & BVAR4 & 4 BVAR4 & BVAR4 & BVAR4 & BVAR4 & BVAR4 & BVAR4 & BVAR4 & $4 \operatorname{VAR}(2)$ \\
\hline 0.95 & BVAR4 & BVAR4 & BVAR4 & BVAR3 & 3 BVAR4 & BVAR4 & BVAR4 & BVAR4 & BVAR4 & BVAR4 & BVAR4 & 4 BVAR4 \\
\hline \multirow[t]{2}{*}{0.99} & BVAR4 & BVAR4 & BVAR4 & BVAR4 & 4 BVAR3 & BVAR3 & BVAR3 & BVAR3 & BVAR4 & BVAR4 & BVAR4 & 4 BVAR4 \\
\hline & \multicolumn{12}{|c|}{ COLLINEARITY $(\rho)$} \\
\hline \multirow{2}{*}{\multicolumn{2}{|c|}{$\begin{array}{c}\text { AUTOCO } \\
\text { LEVELS }(\delta)\end{array}$}} & \multicolumn{2}{|c|}{0.85} & & \multicolumn{2}{|c|}{0.9} & \multicolumn{3}{|c|}{0.95} & \multicolumn{3}{|c|}{0.99} \\
\hline & & RMSE & MAE & & RMSE & MAE & & MSE & MAE & RMS & & MAE \\
\hline-0.99 & & BVAR4 & BVAR & & BVAR4 & BVAR4 & $\mathrm{BV}$ & AR4 & BVAR4 & BVAR & & BVAR4 \\
\hline-0.95 & & BVAR2 & BVAR: & & BVAR2 & BVAR2 & $\mathrm{BV}$ & IAR2 & BVAR2 & BVAF & & BVAR2 \\
\hline-0.9 & & BVAR2 & $\operatorname{VAR}(2$ & & BVAR2 & BVAR2 & $\mathrm{BV}$ & IAR2 & BVAR2 & BVAF & & BVAR2 \\
\hline-0.85 & & $\operatorname{VAR}(2)$ & $\operatorname{VAR}(2$ & & BVAR2 & VAR(2) & $\mathrm{BV}$ & IAR2 & BVAR2 & BVAF & & BVAR2 \\
\hline-0.8 & & VAR(2) & $\operatorname{VAR}(2$ & & $\operatorname{VAR}(2)$ & VAR(2) & VAl & $\mathrm{R}(2)$ & VAR(2) & BVAP & & BVAR2 \\
\hline 0.8 & & VAR(2) & VAR(2 & & $\operatorname{VAR}(2)$ & VAR(2) & VAl & $\mathrm{R}(2)$ & VAR(2) & VAR( & & VAR(2) \\
\hline 0.85 & & VAR(2) & VAR(2 & & $\operatorname{VAR}(2)$ & VAR(2) & VAl & $\mathrm{R}(2)$ & VAR(2) & VAR( & & VAR(2) \\
\hline 0.9 & & BVAR4 & BVAR & & BVAR4 & BVAR4 & $\mathrm{BV}$ & AR4 & BVAR4 & BVAR & & BVAR4 \\
\hline 0.95 & & BVAR4 & BVAR & & BVAR4 & BVAR4 & $\mathrm{BV}$ & AR4 & BVAR4 & BVAR & & BVAR4 \\
\hline 0.99 & & BVAR4 & BVAR & & BVAR4 & BVAR4 & $\mathrm{BV}$ & AR4 & BVAR4 & BVAF & & BVAR4 \\
\hline
\end{tabular}


Table 7. Preferred model at different levels of collinearity and autocorrelation when $T=128$.

\begin{tabular}{|c|c|c|c|c|c|c|c|c|c|c|c|c|}
\hline \multirow{3}{*}{$\begin{array}{c}\text { AUTOCO } \\
\text { LEVELS }(\delta)\end{array}$} & \multicolumn{12}{|c|}{ COLLINERITY $(\rho)$} \\
\hline & \multicolumn{2}{|c|}{-0.99} & \multicolumn{2}{|c|}{-0.95} & \multicolumn{2}{|c|}{-0.9} & \multicolumn{2}{|c|}{-0.85} & \multicolumn{2}{|c|}{-0.8} & \multicolumn{2}{|c|}{0.8} \\
\hline & RMSE & MAE & RMSE & MAE & RMSE & MAE & RMSE & MAE & RMSE & MAE & RMSE & MAE \\
\hline-0.99 & BVAR4 & BVAR4 & BVAR3 & BVAR3 & BVAR3 & BVAR3 & BVAR2 & BVAR2 & BVAR2 & BVAR2 & BVAR4 & BVAR4 \\
\hline-0.95 & BVAR1 & BVAR2 & BVAR1 & BVAR2 & BVAR1 & BVAR1 & BVAR1 & BVAR1 & BVAR1 & BVAR1 & BVAR4 & BVAR4 \\
\hline-0.9 & BVAR2 & BVAR2 & BVAR1 & BVAR1 & BVAR2 & BVAR2 & BVAR4 & BVAR4 & BVAR4 & BVAR4 & VAR(2) & $\operatorname{VAR}(2)$ \\
\hline-0.85 & BVAR1 & BVAR1 & BVAR4 & BVAR4 & BVAR4 & BVAR4 & BVAR4 & BVAR4 & BVAR4 & BVAR4 & VAR(2) & $\operatorname{VAR}(2)$ \\
\hline-0.8 & BVAR2 & BVAR1 & BVAR4 & BVAR4 & BVAR4 & BVAR4 & BVAR4 & BVAR4 & VAR(2) & BVAR4 & VAR(2) & $\operatorname{VAR}(2)$ \\
\hline 0.8 & BVAR4 & BVAR4 & BVAR4 & BVAR4 & BVAR4 & BVAR4 & VAR(2) & VAR(2) & VAR(2) & VAR(2) & VAR(2) & $\operatorname{VAR}(2)$ \\
\hline 0.85 & BVAR4 & BVAR4 & BVAR4 & BVAR4 & BVAR4 & BVAR4 & BVAR4 & BVAR4 & VAR(2) & BVAR4 & VAR(2) & $\operatorname{VAR}(2)$ \\
\hline 0.9 & BVAR4 & BVAR4 & BVAR4 & BVAR4 & BVAR4 & BVAR4 & BVAR4 & BVAR4 & BVAR4 & BVAR4 & VAR(2) & $\operatorname{VAR}(2)$ \\
\hline 0.95 & BVAR4 & BVAR4 & BVAR4 & BVAR4 & BVAR4 & BVAR4 & BVAR4 & BVAR4 & BVAR4 & BVAR4 & BVAR4 & BVAR4 \\
\hline 0.99 & BVAR3 & BVAR3 & BVAR4 & BVAR4 & BVAR3 & BVAR3 & BVAR3 & BVAR3 & BVAR3 & BVAR3 & BVAR3 & BVAR3 \\
\hline
\end{tabular}

\begin{tabular}{|c|c|c|c|c|c|c|c|c|}
\hline \multirow{3}{*}{$\begin{array}{c}\text { AUTOCO } \\
\text { LEVELS }(\delta)\end{array}$} & \multicolumn{8}{|c|}{ COLLINEARITY $(\rho)$} \\
\hline & \multicolumn{2}{|c|}{0.85} & \multicolumn{2}{|c|}{0.9} & \multicolumn{2}{|c|}{0.95} & \multicolumn{2}{|c|}{0.99} \\
\hline & RMSE & MAE & RMSE & MAE & RMSE & MAE & RMSE & MAE \\
\hline-0.99 & BVAR4 & BVAR4 & BVAR4 & BVAR4 & BVAR4 & BVAR4 & BVAR3 & BVAR4 \\
\hline-0.95 & BVAR4 & BVAR4 & BVAR4 & BVAR4 & BVAR2 & BVAR2 & BVAR2 & BVAR2 \\
\hline-0.9 & VAR(2) & VAR(2) & BVAR4 & VAR(2) & BVAR4 & BVAR4 & BVAR2 & BVAR2 \\
\hline-0.85 & VAR(2) & VAR(2) & VAR(2) & VAR(2) & VAR(2) & VAR(2) & BVAR2 & BVAR2 \\
\hline-0.8 & VAR(2) & VAR(2) & VAR(2) & VAR(2) & VAR(2) & $\operatorname{VAR}(2)$ & BVAR2 & BVAR2 \\
\hline 0.8 & VAR(2) & VAR(2) & VAR(2) & VAR(2) & VAR(2) & $\operatorname{VAR}(2)$ & VAR(2) & $\operatorname{VAR}(2)$ \\
\hline 0.85 & VAR(2) & VAR(2) & $\operatorname{VAR}(2)$ & $\operatorname{VAR}(2)$ & $\operatorname{VAR}(2)$ & $\operatorname{VAR}(2)$ & $\operatorname{VAR}(2)$ & $\operatorname{VAR}(2)$ \\
\hline 0.9 & $\operatorname{VAR}(2)$ & $\operatorname{VAR}(2)$ & $\operatorname{VAR}(2)$ & $\operatorname{VAR}(2)$ & BVAR4 & BVAR4 & BVAR4 & BVAR4 \\
\hline 0.95 & BVAR4 & BVAR4 & BVAR4 & BVAR4 & BVAR4 & BVAR4 & BVAR4 & BVAR4 \\
\hline 0.99 & BVAR4 & BVAR4 & BVAR4 & BVAR4 & BVAR4 & BVAR4 & BVAR4 & BVAR4 \\
\hline
\end{tabular}

Table 8. Preferred model at different levels of collinearity and autocorrelation when $T=256$.

\begin{tabular}{|c|c|c|c|c|c|c|c|c|c|c|c|c|}
\hline \multirow{3}{*}{$\begin{array}{c}\text { AUTOCO } \\
\text { LEVELS }(\delta)\end{array}$} & \multicolumn{12}{|c|}{ COLLINERITY $(\rho)$} \\
\hline & \multicolumn{2}{|c|}{-0.99} & \multicolumn{2}{|c|}{-0.95} & \multicolumn{2}{|c|}{-0.9} & \multicolumn{2}{|c|}{-0.85} & \multicolumn{2}{|c|}{-0.8} & \multicolumn{2}{|c|}{0.8} \\
\hline & RMSE & MAE & RMSE & MAE & RMSE & MAE & RMSE & MAE & RMSE & MAE & RMSE & MAE \\
\hline-0.99 & BVAR2 & BVAR2 & BVAR1 & BVAR1 & BVAR2 & BVAR2 & BVAR1 & BVAR1 & BVAR1 & BVAR1 & BVAR4 & BVAR4 \\
\hline-0.95 & BVAR1 & BVAR1 & BVAR1 & BVAR1 & BVAR1 & BVAR1 & BVAR1 & BVAR1 & BVAR1 & BVAR1 & BVAR4 & BVAR4 \\
\hline-0.9 & BVAR1 & BVAR1 & BVAR1 & BVAR1 & BVAR3 & BVAR1 & BVAR2 & BVAR2 & BVAR4 & BVAR4 & VAR(2) & $\operatorname{VAR}(2)$ \\
\hline-0.85 & BVAR2 & BVAR2 & BVAR4 & BVAR4 & BVAR4 & 24 & BVAR4 & BVAR4 & BVAR4 & BVAR4 & VAR(2) & $\operatorname{VAR}(2)$ \\
\hline-0.8 & BVAR1 & BVAR1 & BVAR4 & BVAR4 & BVAR4 & BVAR4 & BVAR4 & BVAR4 & $\operatorname{VAR}(2)$ & VAR(2) & VAR(2) & $\operatorname{VAR}(2)$ \\
\hline 0.8 & BVAR4 & BVAR4 & BVAR4 & BVAR4 & VAR(2) & VAR(2) & VAR(2) & VAR(2) & VAR(2) & VAR(2) & VAR(2) & $\operatorname{VAR}(2)$ \\
\hline 0.85 & BVAR4 & BVAR4 & BVAR4 & BVAR4 & BVAR4 & BVAR4 & VAR(2) & VAR(2) & VAR(2) & VAR(2) & VAR(2) & $\operatorname{VAR}(2)$ \\
\hline 0.9 & BVAR4 & BVAR4 & BVAR4 & BVAR4 & BVAR4 & BVAR4 & BVAR4 & BVAR4 & BVAR4 & BVAR4 & VAR(2) & $\operatorname{VAR}(2)$ \\
\hline 0.95 & BVAR4 & BVAR4 & BVAR4 & BVAR3 & BVAR4 & BVAR4 & BVAR4 & BVAR4 & BVAR4 & BVAR4 & BVAR4 & BVAR4 \\
\hline 0.99 & BVAR4 & BVAR4 & BVAR3 & BVAR3 & BVAR1 & BVAR1 & BVAR3 & BVAR3 & BVAR3 & BVAR3 & BVAR4 & BVAR4 \\
\hline
\end{tabular}




\begin{tabular}{|c|c|c|c|c|c|c|c|c|}
\hline \multirow{3}{*}{$\begin{array}{c}\text { AUTOCO } \\
\text { LEVELS }(\delta)\end{array}$} & \multicolumn{8}{|c|}{ COLLINEARITY $(\rho)$} \\
\hline & \multicolumn{2}{|c|}{0.85} & \multicolumn{2}{|c|}{0.9} & \multicolumn{2}{|c|}{0.95} & \multicolumn{2}{|c|}{0.99} \\
\hline & RMSE & MAE & RMSE & MAE & RMSE & MAE & RMSE & MAE \\
\hline-0.99 & BVAR2 & BVAR4 & BVAR3 & BVAR4 & BVAR2 & BVAR2 & BVAR1 & BVAR1 \\
\hline-0.95 & BVAR4 & BVAR4 & BVAR4 & BVAR4 & BVAR2 & BVAR2 & BVAR2 & BVAR2 \\
\hline-0.9 & VAR(2) & $\operatorname{VAR}(2)$ & VAR(2) & $\operatorname{VAR}(2)$ & BVAR4 & VAR(2) & BVAR2 & BVAR4 \\
\hline-0.85 & VAR(2) & VAR(2) & VAR(2) & VAR(2) & VAR(2) & VAR(2) & BVAR2 & BVAR4 \\
\hline-0.8 & VAR(2) & VAR(2) & VAR(2) & VAR(2) & VAR(2) & VAR(2) & BVAR4 & $\operatorname{VAR}(2)$ \\
\hline 0.8 & VAR(2) & VAR(2) & VAR(2) & VAR(2) & VAR(2) & VAR(2) & VAR(2) & $\operatorname{VAR}(2)$ \\
\hline 0.85 & VAR(2) & VAR(2) & VAR(2) & VAR(2) & VAR(2) & VAR(2) & VAR(2) & $\operatorname{VAR}(2)$ \\
\hline 0.9 & VAR(2) & VAR(2) & VAR(2) & VAR(2) & VAR(2) & VAR(2) & BVAR4 & BVAR4 \\
\hline 0.95 & BVAR4 & BVAR4 & BVAR4 & BVAR4 & BVAR4 & BVAR4 & BVAR4 & BVAR4 \\
\hline 0.99 & BVAR4 & BVAR4 & BVAR4 & BVAR4 & BVAR4 & BVAR4 & BVAR3 & BVAR3 \\
\hline
\end{tabular}

Table 9. Performance ratings of the classical VAR and Sims-Zha Bayesian VAR.

\begin{tabular}{|c|c|c|c|c|c|c|c|c|c|c|c|c|}
\hline \multirow[b]{3}{*}{ Models } & \multicolumn{4}{|c|}{ Short Term } & \multicolumn{4}{|c|}{ Medium Term } & \multicolumn{4}{|c|}{ Long Term } \\
\hline & \multicolumn{2}{|c|}{$T=8$} & \multicolumn{2}{|c|}{$T=16$} & \multicolumn{2}{|c|}{$T=32$} & \multicolumn{2}{|c|}{$T=64$} & \multicolumn{2}{|c|}{$T=128$} & \multicolumn{2}{|c|}{$T=256$} \\
\hline & RMSE & MAE & RMSE & MAE & RMSE & MAE & RMSE & MAE & RMSE & MAE & RMSE & MAE \\
\hline VAR(2) & - & - & - & - & $5 \%$ & $11 \%$ & $16 \%$ & $20 \%$ & $27 \%$ & $26 \%$ & $31 \%$ & $33 \%$ \\
\hline BVAR1 & $37 \%$ & $53 \%$ & $20 \%$ & $24 \%$ & - & - & $1 \%$ & $3 \%$ & $7 \%$ & $6 \%$ & $13 \%$ & $14 \%$ \\
\hline BVAR2 & $7 \%$ & $2 \%$ & $9 \%$ & $14 \%$ & $15 \%$ & $12 \%$ & $20 \%$ & $18 \%$ & $10 \%$ & $11 \%$ & $10 \%$ & $7 \%$ \\
\hline BVAR3 & $12 \%$ & $11 \%$ & $6 \%$ & $4 \%$ & $6 \%$ & $8 \%$ & $2 \%$ & $4 \%$ & $7 \%$ & $7 \%$ & $6 \%$ & $5 \%$ \\
\hline BVAR4 & $44 \%$ & $34 \%$ & $65 \%$ & $58 \%$ & $74 \%$ & $69 \%$ & $61 \%$ & $55 \%$ & $49 \%$ & $50 \%$ & $40 \%$ & $41 \%$ \\
\hline
\end{tabular}

In Table 9 above, the ratings of the model are compared. For short term time series the BVAR4 model dominate except when $T=8$ using the MAE criterion, the BVAR1 model dominate. In the medium term and long term, the BVAR4 model dominated using both criteria. This result revealed that the BVAR4 model is a viable model for forecasting.

\section{Conclusion and Recommendation}

This work examines the performances of classical VAR and Sims-Zha Bayesian VAR in the presence of collinearity and autocorrelation. The results from 10,000 simulations reveal that the models performance varies with the collinearity and autocorrelation levels, and with the time series lengths. In addition, the results reveal that the BVAR4 model is a viable model for forecasting. Therefore, we recommend that the levels of collinearity and autocorrelation, and the time series length should be considered in using an appropriate model for forecasting.

\section{Acknowledgements}

We wish to thank TETFUND Abuja-Nigeria for sponsoring this research work. Our appreciation also goes to the Rector and the Directorate of Research, Conference and Publication of the Federal Polytechnic Bida for giving us this opportunity to undergo this research work.

\section{References}

[1] Brillinger, D.R. (2000) Time Series: General. University of California, Berkeley.

[2] Gujarati, D.N. (2003) Basic Econometrics. 4th Edition, The McGraw-Hill Co., New Delhi.

[3] Dormann, C.F., Elith, J., Bacher, S., Bachmann, C., Carl, G., Lagourcade, B., Leifao, P.J., Munkemiller, T., McClean, C., Osborne, P.E., Reineking, B., Schroder, B., Skidmore, A.K., Jurell, D. and Lautenbach, S. (2013) Collinearity: A 
Review of Methods to Deal with It and A Simulation Study Evaluating their Performance. Ecography, 36, 027-046. http://dx.doi.org/10.1111/j.1600-0587.2012.07348.x

[4] Garba, M.K., Oyejola, B.A. and Yahya, W.B. (2013) Investigations of Certain Estimators for Modelling Panel Data under Violations of Some Basic Assumptions. Mathematical Theory and Modeling, 3, 47-53.

[5] Adenomon, M.O. and Oyejola, B.A. (2014) Forecasting Performances of the Reduced Form VAR and Sims-Zha Bayesian VAR Models When the Multiple Times Series Are Jointly Influence by Collinearity and Autocorrelation. A Paper Presented at the 1st Int'l Conference on Postgraduate Students' Association, University of Ilorin, Ilorin, Kwara State, Nigeria, 14-16 October 2014.

[6] Lutkepohl, H. and Breitung, J. (1996) Impulse Response Analysis of Vector Autoregressive Processes: System Dynamic in Economic and Financial Models. SFB 373 Discussion Papers 1996, 86, Humboldt University of Berlin, Interdisciplinary Research Project 373: Quantification and Simulation of Economic Processes.

[7] Yang, M. (2002) Lag Length and Mean Break in Stationary VAR Models. The Econometrics Journal, 5, 374-386. http://dx.doi.org/10.1111/1368-423X.00089

[8] Sims, C.A. and Zha, T. (1998) Bayesian Methods for Dynamic Multivariate Models. International Economic Review, 39, 949-968. http://dx.doi.org/10.2307/2527347

[9] Brandt, P.T. and Freeman, J.R. (2009) Modeling Macro-Political Dynamics. Political Analysis, 17, 113-142. http://dx.doi.org/10.1093/pan/mpp001

[10] Brandt, P.T. and Freeman, J.R. (2006) Advances in Bayesian Time Series Modeling and the Study of Politics: Theory, Testing, Forecasting and Policy Analysis. Political Analysis, 14, 1-36. http://dx.doi.org/10.1093/pan/mpi035

[11] Sims, C.A. and Zha, T. (1999) Error Bands for Impulse Responses. Econometrica, 67, 1113-1155. http://dx.doi.org/10.1111/1468-0262.00071

[12] Cowpertwait, P.S.P. (2006) Introductory Time Series with R. SPRINGER SCIENCE+BUSINESS MEDIA, LLC. New York.

[13] Caraiani, P. (2010) Forecasting Romanian GDP Using A BVAR Model. Romanian Journal of Economic Forecasting, 4, 76-87.

[14] Diebold, F.X. and Mariano, R.S. (2002) Comparing Predictive Accuracy. Journal of Business and Economic Statistics, 20, 134-144. http://dx.doi.org/10.1198/073500102753410444

[15] Kadiyala, K.R. and Karlsson, S. (1997) Numerical Methods for Estimation and Inference in Bayesian VAR Models. Journal of Applied Econometrics, 12, 99-132. http://dx.doi.org/10.1002/(SICI)1099-1255(199703)12:2<99::AID-JAE429>3.0.CO;2-A

[16] Breheny, P. (2013) Wishart Priors. BST 701: Bayesian Modelling in Biostatistics. http://web.as.uky.edu/statistics/users/pbreheny/701/S13/notes/3-28.pdf

[17] Brandt, P.T., Colaresi, M. and Freeman, J.R. (2008) Dynamic of Reciprocity, Accountability and Credibility. Journal of Conflict Resolution, 52, 343-374. http://dx.doi.org/10.1177/0022002708314221

[18] Merkin, J.H. and Needman, D.J. (1990) The Development of Travelling Waves in a Simple Isothermal Chemical System II. Cubic Autocatalysis with Quadratic and Linear Decay. Proceedings: Mathematical \& Physical Sciences, 430, 315-345.

[19] Merkin, J.H. and Needman, D.J. (1991) The Development of Travelling Waves in a Simple Isothermal Chemical System IV. Quadratic Autocatalysis with Quadratic Decay. Proceedings: Mathematical \& Physical Sciences, 434, 531554.

[20] Worsley, K.J., Evans, A.C., Strother, S.C. and Tyler, J.L. (1991) A Linear Spatial Correlation Model with Applications to Positron Emission Tomography. Journal of the American Statistical Association, 86, 55-67. http://dx.doi.org/10.1080/01621459.1991.10475004

[21] Cooray, T. M.J.A. (2008) Applied Time Series Analysis and Forecasting. Narosa Publising House, New Delhi.

[22] Gilbert, P. (2009) Brief User's Guide: Dynamic Systems Estimation (DSE). www.bank-banque-canada.ca/pgilbert

[23] Pfaff, B. (2008) VAR, SVAR and SVEC Models: Implementation within R Package Vars. Journal of Statistical Software, 27, 1-32. http://dx.doi.org/10.18637/jss.v027.i04

[24] Brandt, P.T. (2012) Markov-Switching, Bayesian Vector Autoregression Models-Package “MSBVAR”. The R Foundation for Statistical Computing. 


\section{Appendix A}

Table A1. The RMSE and MAE values of the model for $T=8$.

\begin{tabular}{|c|c|c|c|c|c|c|c|c|c|c|c|c|c|}
\hline$T=8$ & & & & & & & COLLINE & RITY $(\rho)$ & & & & & \\
\hline \multirow{2}{*}{$\begin{array}{c}\text { AUTOCO } \\
\text { LEVELS }(\delta)\end{array}$} & \multirow[t]{2}{*}{ Models } & \multicolumn{2}{|c|}{-0.99} & \multicolumn{2}{|c|}{-0.95} & \multicolumn{2}{|c|}{-0.9} & \multicolumn{2}{|c|}{-0.85} & \multicolumn{2}{|c|}{-0.8} & \multicolumn{2}{|c|}{0.8} \\
\hline & & RMSE & MAE & RMSE & MAE & RMSE & MAE & RMSE & MAE & RMSE & MAE & RMSE & MAE \\
\hline \multirow{5}{*}{-0.99} & VAR(2) & N/A & N/A & N/A & N/A & N/A & N/A & N/A & N/A & N/A & N/A & N/A & N/A \\
\hline & BVAR1 & 5.568972 & 4.357228 & 6.346049 & 4.906742 & 6.791074 & 5.226318 & 6.893263 & 5.304391 & 6.930938 & 5.378541 & 4.846500 & 3.749898 \\
\hline & BVAR2 & 5.471007 & 4.306492 & 6.151134 & 4.805023 & 6.519096 & 5.070815 & 6.713149 & 5.260478 & 6.650402 & 5.252652 & 4.806706 & 3.741190 \\
\hline & BVAR3 & 4.995865 & 4.107761 & 5.510815 & 4.545857 & 5.737559 & 4.752087 & 5.927203 & 4.932999 & 6.004684 & 4.990791 & 4.586969 & 3.749766 \\
\hline & BVAR4 & 4.913680 & 4.054765 & 5.337825 & 4.442210 & 5.571354 & 4.654506 & 5.696384 & 4.751056 & 5.789108 & 4.793418 & 4.548321 & 3.755239 \\
\hline
\end{tabular}

\begin{tabular}{|c|c|c|c|c|c|c|c|c|c|c|c|c|c|}
\hline & VAR(2) & N/A & N/A & N/A & N/A & N.A & N/A & N/A & N/A & N/A & N/A & N/A & N/A \\
\hline & BVAR1 & 4.924112 & 3.811504 & 5.514037 & 4.239658 & 5.869246 & 4.486138 & 6.038599 & 4.618446 & 6.084726 & 4.694254 & 4.302530 & 3.300939 \\
\hline \multirow[t]{3}{*}{-0.95} & BVAR2 & 4.894266 & 3.809230 & 5.419252 & 4.193870 & 5.667241 & 4.371583 & 5.914171 & 4.594582 & 5.841484 & 4.577024 & 4.274452 & 3.297456 \\
\hline & BVAR3 & 4.552996 & 3.663140 & 4.967818 & 4.020410 & 5.178609 & 4.219038 & 5.315171 & 4.352908 & 5.305581 & 4.340806 & 4.224433 & 3.406297 \\
\hline & BVAR4 & 4.509209 & 3.639075 & 4.804357 & 3.903115 & 4.988811 & 4.086084 & 5.110920 & 4.202868 & 5.201375 & 4.266454 & 4.233088 & 3.446089 \\
\hline
\end{tabular}

$\begin{array}{llllllllllllll}\text { VAR(2) N/A } & \text { N/A } & \text { N/A } & \text { N/A } & \text { N/A } & \text { N/A } & \text { N/A } & \text { N/A } & \text { N/A } & \text { N/A } & \text { N/A } & \text { N/A }\end{array}$

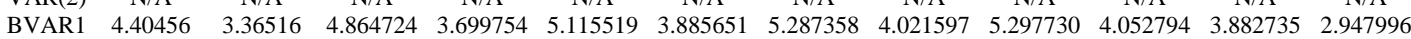
$\begin{array}{llllllllllllll}\text { BVAR2 } & 4.421230 & 3.393082 & 4.830294 & 3.695425 & 5.050976 & 3.860234 & 5.142196 & 3.946026 & 5.164368 & 3.998000 & 3.873145 & 2.957112\end{array}$ $\begin{array}{llllllllllllll}\text { BVAR3 } & 4.206626 & 3.303933 & 4.473879 & 3.529379 & 4.647730 & 3.690321 & 4.766993 & 3.816559 & 4.807192 & 3.862643 & 3.922100 & 3.129902\end{array}$

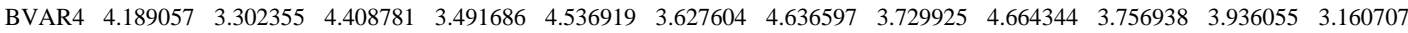

\begin{tabular}{|c|c|c|c|c|c|c|c|c|c|c|c|c|c|}
\hline & VAR(2) & N/A & N/A & N/A & N/A & N/A & N/A & N/A & N/A & N/A & N/A & N/A & N/A \\
\hline \multirow{4}{*}{-0.85} & BVAR1 & 4.172696 & 3.150146 & 4.460275 & 3.361247 & 4.673119 & 3.524658 & 4.791012 & 3.615496 & 4.828145 & 3.665550 & 3.617484 & 2.72217 \\
\hline & BVAR2 & 4.144112 & 3.146404 & 4.459834 & 3.381266 & 4.630900 & 3.506207 & 4.693236 & 3.561376 & 4.736905 & 3.634963 & 3.621705 & $2.7381 \mathrm{C}$ \\
\hline & BVAR3 & 4.002858 & 3.082628 & 4.199869 & 3.248724 & 4.325427 & 3.368202 & 4.411859 & 3.463734 & 4.45633 & 3.51665 & 3.745665 & 2.964287 \\
\hline & BVAR4 & 3.964532 & 3.055228 & 4.160505 & 3.230431 & 4.282877 & 3.353908 & 4.308728 & 3.402976 & 4.331752 & 3.426619 & 3.788757 & 3.01865 \\
\hline
\end{tabular}

\begin{tabular}{|c|c|c|c|c|c|c|c|c|c|c|c|c|c|}
\hline \multirow{5}{*}{-0.8} & VAR(2) & N/A & N/A & N/A & N/A & N/A & N/A & N/A & N/A & N/A & N/A & N/A & N/A \\
\hline & BVAR1 & 3.966541 & 2.963655 & 4.212467 & 3.146166 & 4.386190 & 3.277238 & 4.475193 & 3.354209 & 4.512634 & 3.394073 & 3.446991 & 2.570778 \\
\hline & BVAR2 & 3.982988 & 2.986436 & 4.192779 & 3.136875 & 4.359005 & 3.275833 & 4.445047 & 3.345427 & 4.453733 & 3.380426 & 3.457457 & 2.593641 \\
\hline & BVAR3 & 3.859199 & 2.928322 & 4.020930 & 3.058512 & 4.138915 & 3.174228 & 4.180315 & 3.229568 & 4.222451 & 3.285421 & 3.624815 & 2.857300 \\
\hline & BVAR4 & 3.865623 & 2.939495 & 3.977014 & 3.027379 & 4.060440 & 3.120372 & 4.088982 & 3.165973 & 4.135093 & 3.228313 & 3.667822 & 2.911481 \\
\hline
\end{tabular}
$\begin{array}{lllllllllllll}\text { VAR(2) N/A } & \text { N/A } & \text { N/A } & \text { N/A } & \text { N/A } & \text { N/A } & \text { N/A } & \text { N/A } & \text { N/A } & \text { N/A } & \text { N/A } & \text { N/A }\end{array}$

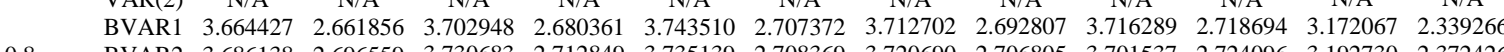
$\begin{array}{llllllllllllll}\text { BVAR2 } & 3.664427 & 2.661856 & 3.702948 & 2.680361 & 3.743510 & 2.707372 & 3.712702 & 2.692807 & 3.716289 & 2.718694 & 3.172067 & 2.339266 \\ \text { BVAR2 } & 3.686138 & 2.696559 & 3.730683 & 2.712849 & 3.735139 & 2.708369 & 3.720690 & 2.706805 & 3.701537 & 2.724096 & 3.192730 & 2.372426\end{array}$ $\begin{array}{llllllllllllll}\text { BVAR3 } & 3.671995 & 2.698841 & 3.668494 & 2.679722 & 3.664705 & 2.673309 & 3.661278 & 2.687255 & 3.626062 & 2.680750 & 3.418707 & 2.698835\end{array}$

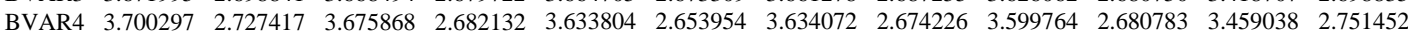

\begin{tabular}{|c|c|c|c|c|c|c|c|c|c|c|c|c|c|}
\hline \multirow{5}{*}{0.85} & VAR(2) & N/A & N/A & N/A & N/A & N/A & N/A & N/A & N/A & N/A & N/A & N/A & N/A \\
\hline & BVAR1 & 3.705972 & 2.704436 & 3.748004 & 2.721802 & 3.745347 & 2.714481 & 3.750389 & 2.725927 & 3.729666 & 2.734343 & 3.186470 & 2.348993 \\
\hline & BVAR2 & 3.702085 & 2.706138 & 3.752680 & 2.732167 & 3.738364 & 2.716470 & 3.736367 & 2.726194 & 3.751587 & 2.773472 & 3.213187 & 2.383334 \\
\hline & BVAR3 & 3.700710 & 2.722275 & 3.698570 & 2.709008 & 3.695413 & 2.704197 & 3.664489 & 2.693061 & 3.653480 & 2.715004 & 3.405944 & 2.680517 \\
\hline & BVAR4 & 3.720387 & 2.750880 & 3.701877 & 2.714742 & 3.679862 & 2.701747 & 3.651776 & 2.696512 & 3.654069 & 2.736138 & 3.469572 & 2.756269 \\
\hline \multirow{5}{*}{0.9} & VAR(2) & N/A & N/A & N/A & N/A & N/A & N/A & N/A & N/A & N/A & N/A & N/A & N/A \\
\hline & BVAR1 & 3.695506 & 2.694242 & 3.756887 & 2.729671 & 3.791675 & 2.759002 & 3.777360 & 2.757002 & 3.766396 & 2.774918 & 3.200669 & 2.359688 \\
\hline & BVAR2 & 3.713280 & 2.721317 & 3.762058 & 2.742930 & 3.751100 & 2.734233 & 3.779886 & 2.767498 & 3.777727 & 2.804381 & 3.201165 & 2.375459 \\
\hline & BVAR3 & 3.717683 & 2.749170 & 3.723994 & 2.731201 & 3.708352 & 2.719700 & 3.694109 & 2.724118 & 3.690586 & 2.746572 & 3.428952 & 2.697854 \\
\hline & BVAR4 & 3.731138 & 2.763758 & 3.697047 & 2.711482 & 3.699408 & 2.719445 & 3.680536 & 2.723904 & 3.675044 & 2.756350 & 3.459280 & 2.743717 \\
\hline \multirow{5}{*}{0.95} & VAR(2) & N/A & N/A & N/A & N/A & N/A & N/A & N/A & N/A & N/A & N/A & N/A & N/A \\
\hline & BVAR1 & 3.722003 & 2.721109 & 3.794420 & 2.761852 & 3.768156 & 2.745364 & 3.782314 & 2.761302 & 3.783861 & 2.795752 & 3.203982 & 2.358522 \\
\hline & BVAR2 & 3.724179 & 2.732423 & 3.768054 & 2.750236 & 3.789197 & 2.767812 & 3.800848 & 2.791411 & 3.800298 & 2.824817 & 3.225113 & 2.393398 \\
\hline & BVAR3 & 3.717546 & 2.744508 & 3.726093 & 2.731677 & 3.714950 & 2.727748 & 3.716211 & 2.749526 & 3.721622 & 2.782934 & 3.429190 & 2.692399 \\
\hline & BVAR4 & 3.743243 & 2.776283 & 3.741126 & 2.755417 & 3.694183 & 2.716774 & 3.696180 & 2.745173 & 3.693516 & 2.774400 & 3.477101 & 2.751985 \\
\hline \multirow{5}{*}{0.99} & VAR(2) & N/A & N/A & N/A & N/A & N/A & N/A & N/A & N/A & N/A & N/A & N/A & N/A \\
\hline & BVAR1 & 3.717622 & 2.714390 & 3.792705 & 2.767841 & 3.798213 & 2.767834 & 3.789347 & 2.768060 & 3.815843 & 2.824166 & 3.220688 & 2.372169 \\
\hline & BVAR2 & 3.735449 & 2.744270 & 3.775062 & 2.758082 & 3.791127 & 2.768266 & 3.783690 & 2.776671 & 3.820224 & 2.845886 & 3.242346 & 2.406335 \\
\hline & BVAR3 & 3.719875 & 2.748584 & 3.739040 & 2.743129 & 3.728527 & 2.739729 & 3.732343 & 2.764869 & 3.722467 & 2.782521 & 3.434744 & 2.689321 \\
\hline & BVAR4 & 3.740953 & 2.770389 & 3.736597 & 2.752376 & 3.722800 & 2.745257 & 3.722079 & 2.772045 & 3.697656 & 2.780737 & 3.471326 & 2.736448 \\
\hline
\end{tabular}




\section{Continued}

\begin{tabular}{|c|c|c|c|c|c|c|c|c|c|}
\hline \multirow{3}{*}{$\begin{array}{c}\text { AUTOCO } \\
\text { LEVELS }(\delta)\end{array}$} & \multirow{3}{*}{ Models } & \multicolumn{8}{|c|}{ COLLINEARITY $(\rho)$} \\
\hline & & \multicolumn{2}{|c|}{0.85} & \multicolumn{2}{|c|}{0.9} & \multicolumn{2}{|c|}{0.95} & \multicolumn{2}{|c|}{0.99} \\
\hline & & RMSE & MAE & RMSE & MAE & RMSE & MAE & RMSE & MAE \\
\hline \multirow{5}{*}{-0.99} & VAR(2) & N/A & N/A & N/A & N/A & N/A & N/A & N/A & N/A \\
\hline & BVAR1 & 4.537550 & 3.481325 & 4.311283 & 3.222246 & 4.184486 & 3.023109 & 4.462124 & 3.411701 \\
\hline & BVAR2 & 4.558609 & 3.520800 & 4.307658 & 3.239499 & 4.183656 & 3.042939 & 4.456530 & 3.436138 \\
\hline & BVAR3 & 4.394162 & 3.557580 & 4.217586 & 3.334594 & 4.158514 & 3.212328 & 4.282317 & 3.426102 \\
\hline & BVAR4 & 4.358627 & 3.554616 & 4.225348 & 3.373656 & 4.156735 & 3.244152 & 4.254922 & 3.411702 \\
\hline \multirow{5}{*}{-0.95} & VAR(2) & N/A & N/A & N/A & N/A & N/A & N/A & N/A & N/A \\
\hline & BVAR1 & 4.110707 & 3.124814 & 3.937992 & 2.925000 & 3.887423 & 2.820268 & 4.103056 & 3.106144 \\
\hline & BVAR2 & 4.112315 & 3.143094 & 3.938351 & 2.944167 & 3.891292 & 2.847463 & 4.098306 & 3.127103 \\
\hline & BVAR3 & 4.082247 & 3.265733 & 3.976808 & 3.124123 & 3.925936 & 3.033313 & 4.037778 & 3.185102 \\
\hline & BVAR4 & 4.105616 & 3.309773 & 4.002506 & 3.172131 & 3.954466 & 3.080437 & 4.051955 & 3.207187 \\
\hline \multirow{5}{*}{-0.9} & VAR(2) & N/A & N/A & N/A & N/A & N/A & N/A & N/A & N/A \\
\hline & BVAR1 & 3.741569 & 2.812975 & 3.682325 & 2.714770 & 3.645236 & 2.650995 & 3.840964 & 2.877191 \\
\hline & BVAR2 & 3.762917 & 2.848642 & 3.678935 & 2.734708 & 3.662074 & 2.683155 & 3.839072 & 2.897901 \\
\hline & BVAR3 & 3.839031 & 3.040055 & 3.780936 & 2.954366 & 3.751349 & 2.889068 & 3.835362 & 2.978061 \\
\hline & BVAR4 & 3.864931 & 3.082028 & 3.811408 & 3.000603 & 3.803668 & 2.956136 & 3.857246 & 3.008656 \\
\hline \multirow{5}{*}{-0.85} & VAR(2) & N/A & N/A & N/A & N/A & N/A & N/A & N/A & N/A \\
\hline & BVAR1 & 3.527073 & 2.625006 & 3.503651 & 2.570472 & 3.532477 & 2.568397 & 3.676042 & 2.729773 \\
\hline & BVAR2 & 3.544249 & 2.654888 & 3.503690 & 2.587308 & 3.529305 & 2.584525 & 3.679151 & 2.745294 \\
\hline & BVAR3 & 3.695972 & 2.904785 & 3.651980 & 2.837787 & 3.645202 & 2.801735 & 3.719638 & 2.853858 \\
\hline & BVAR4 & 3.712037 & 2.939218 & 3.708709 & 2.911632 & 3.696717 & 2.863443 & 3.784225 & 2.921798 \\
\hline \multirow{5}{*}{-0.8} & VAR(2) & N/A & N/A & N/A & N/A & N/A & N/A & N/A & N/A \\
\hline & BVAR1 & 3.404053 & 2.511238 & 3.398815 & 2.480924 & 3.449632 & 2.501783 & 3.600622 & 2.651085 \\
\hline & BVAR2 & 3.414053 & 2.538967 & 3.415838 & 2.509690 & 3.450560 & 2.520839 & 3.590963 & 2.661195 \\
\hline & BVAR3 & 3.592165 & 2.809641 & 3.572807 & 2.768130 & 3.589777 & 2.746322 & 3.652788 & 2.780575 \\
\hline & BVAR4 & 3.628137 & 2.864632 & 3.627878 & 2.838415 & 3.648316 & 2.821996 & 3.705193 & 2.838146 \\
\hline \multirow{5}{*}{0.8} & VAR(2) & N/A & N/A & N/A & N/A & N/A & N/A & N/A & N/A \\
\hline & BVAR1 & 3.246592 & 2.399710 & 3.310064 & 2.453432 & 3.418468 & 2.528314 & 3.553209 & 2.611308 \\
\hline & BVAR2 & 3.246896 & 2.414905 & 3.33611 & 2.48113 & 3.430743 & 2.550211 & 3.566502 & 2.636769 \\
\hline & BVAR3 & 3.474274 & 2.738317 & 3.488536 & 2.725103 & 3.548691 & 2.732163 & 3.604319 & 2.723500 \\
\hline & BVAR4 & 3.491501 & 2.768120 & 3.560204 & 2.805069 & 3.593645 & 2.793134 & 3.670657 & 2.802106 \\
\hline \multirow{5}{*}{0.85} & VAR(2) & N/A & N/A & N/A & N/A & N/A & N/A & N/A & N/A \\
\hline & BVAR1 & 3.244825 & 2.399588 & 3.326252 & 2.465507 & 3.422633 & 2.530439 & 3.546281 & 2.607774 \\
\hline & BVAR2 & 3.259229 & 2.428672 & 3.354776 & 2.500740 & 3.43176 & 2.54816 & 3.563459 & 2.635383 \\
\hline & BVAR3 & 3.460362 & 2.721740 & 3.495306 & 2.728309 & 3.575695 & 2.758226 & 3.631831 & 2.750430 \\
\hline & BVAR4 & 3.507471 & 2.779017 & 3.554798 & 2.803503 & 3.627435 & 2.819402 & 3.681626 & 2.800921 \\
\hline \multirow{5}{*}{0.9} & VAR(2) & N/A & N/A & N/A & N/A & N/A & N/A & N/A & N/A \\
\hline & BVAR1 & 3.250787 & 2.403782 & 3.346172 & 2.477368 & 3.447617 & 2.550183 & 3.555696 & 2.614251 \\
\hline & BVAR2 & 3.280845 & 2.444396 & 3.341598 & 2.486364 & 3.438260 & 2.557878 & 3.557280 & 2.633103 \\
\hline & BVAR3 & 3.450879 & 2.710523 & 3.505097 & 2.731947 & 3.585112 & 2.761068 & 3.645190 & 2.756749 \\
\hline & BVAR4 & 3.512181 & 2.777651 & 3.544039 & 2.787914 & 3.644604 & 2.829573 & 3.682221 & 2.806372 \\
\hline \multirow{5}{*}{0.95} & VAR(2) & N/A & N/A & N/A & N/A & N/A & N/A & $\mathrm{N} / \mathrm{A}$ & N/A \\
\hline & BVAR1 & 3.262315 & 2.406743 & 3.333332 & 2.467128 & 3.456961 & 2.556855 & 3.559891 & 2.624751 \\
\hline & BVAR2 & 3.295988 & 2.454660 & 3.366857 & 2.513706 & 3.475588 & 2.583734 & 3.587850 & 2.657633 \\
\hline & BVAR3 & 3.474000 & 2.721437 & 3.526608 & 2.747001 & 3.588376 & 2.761531 & 3.656173 & 2.761867 \\
\hline & BVAR4 & 3.502802 & 2.764816 & 3.565433 & 2.797299 & 3.622213 & 2.814620 & 3.693817 & 2.812096 \\
\hline \multirow{5}{*}{0.99} & VAR(2) & N/A & N/A & N/A & N/A & N/A & N/A & N/A & N/A \\
\hline & BVAR1 & 3.286196 & 2.432307 & 3.344424 & 2.473893 & 3.445813 & 2.550056 & 3.580162 & 2.636090 \\
\hline & BVAR2 & 3.284000 & 2.441861 & 3.371049 & 2.512374 & 3.476678 & 2.594105 & 3.582766 & 2.657574 \\
\hline & BVAR3 & 3.476431 & 2.720465 & 3.515471 & 2.733234 & 3.581961 & 2.751931 & 3.643776 & 2.754464 \\
\hline & BVAR4 & 3.530036 & 2.784255 & 3.583805 & 2.809573 & 3.646085 & 2.829619 & 3.726193 & 2.840205 \\
\hline
\end{tabular}


Table A2. The RMSE and MAE values of the model for $T=16$.

\begin{tabular}{|c|c|c|c|c|c|c|c|c|c|c|c|c|c|}
\hline$T=16$ & & & & & & & COLLII & $\operatorname{RITY}(\rho)$ & & & & & \\
\hline AUTOCO & Models & & & & & & & & & & & 0 & \\
\hline (d) & & RMSE & MAE & RMSE & MAE & RMSE & MAE & RMSE & MAE & RMSE & MAE & RMSE & MAE \\
\hline
\end{tabular}

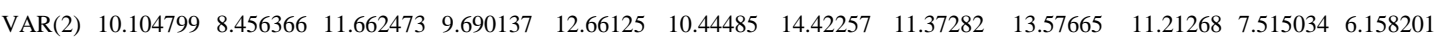
$\begin{array}{lllllllllllllll}\text { BVAR1 } & 7.057613 & 6.177713 & 7.849259 & 6.829913 & 8.272915 & 7.127584 & 8.547135 & 7.257813 & 8.833861 & 7.381579 & 5.702785 & 4.801708\end{array}$ $\begin{array}{lllllllllllllll}\text { BVAR2 } & 6.738948 & 5.953202 & 7.508877 & 6.566234 & 7.981810 & 6.867741 & 8.477262 & 7.170677 & 8.768585 & 7.305375 & 5.521963 & 4.715452\end{array}$ $\begin{array}{lllllllllllllll}\text { BVAR3 } & 6.184788 & 5.463797 & 6.977209 & 6.084434 & 7.570095 & 6.488661 & 8.013359 & 6.761561 & 8.396624 & 6.992618 & 5.150044 & 4.442723\end{array}$ $\begin{array}{lllllllllllll}\text { BVAR4 } & 6.050583 & 5.331047 & 6.880579 & 5.968021 & 7.493272 & 6.370001 & 7.815218 & 6.536759 & 8.124370 & 6.704089 & 5.106301 & 4.370440\end{array}$

\begin{tabular}{rlllllllllllll}
\hline & & & & & & & & & & & & & \\
7 & VAR(2) & 7.678307 & 6.211153 & 8.303226 & 6.793905 & 11.308509 & 8.339395 & 9.527971 & 7.683493 & 11.21796 & 8.52040 & 5.624492 & 4.435031 \\
-0.95 & BVAR1 & 5.505853 & 4.667212 & 6.084709 & 5.154172 & 6.369825 & 5.378279 & 6.515062 & 5.452414 & 6.709748 & 5.533295 & 4.536764 & 3.685262 \\
& BVAR2 & 5.319160 & 4.553144 & 5.828086 & 4.968387 & 6.120845 & 5.171722 & 6.334533 & 5.276264 & 6.613226 & 5.425574 & 4.407507 & 3.621460 \\
& BVAR3 & 4.878560 & 4.180554 & 5.398251 & 4.619672 & 5.729895 & 4.841492 & 5.983213 & 4.982744 & 6.283266 & 5.171398 & 4.20787 & 3.52632 \\
& BVAR4 & 4.810342 & 4.123814 & 5.284490 & 4.508032 & 5.661293 & 4.765141 & 5.980158 & 4.961985 & 6.212964 & 5.091025 & 4.220202 & 3.526784
\end{tabular}

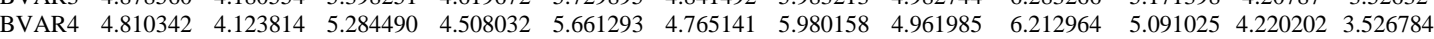

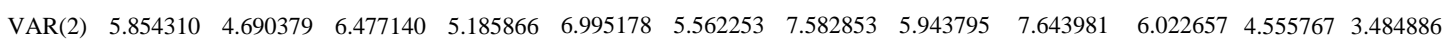

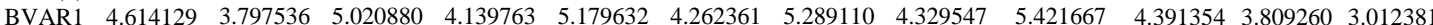
$\begin{array}{lllllllllllll}\text { BVAR2 } & 4.450614 & 3.687862 & 4.820959 & 3.992000 & 5.058707 & 4.175689 & 5.184678 & 4.232059 & 5.334255 & 4.297975 & 3.751630 & 2.991785\end{array}$ $\begin{array}{llllllllllllll}\text { BVAR3 } & 4.104153 & 3.377469 & 4.400405 & 3.644523 & 4.642098 & 3.825978 & 4.854366 & 3.963737 & 5.065288 & 4.098932 & 3.675500 & 3.009166\end{array}$

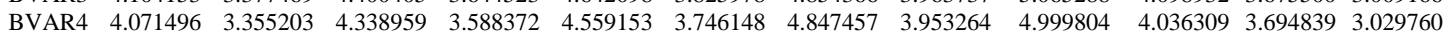

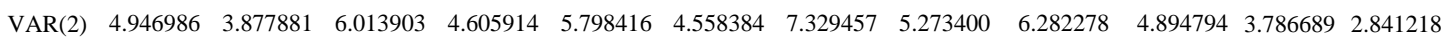
$\begin{array}{lllllllllllll}\text { BVAR1 } & 4.109101 & 3.311935 & 4.413881 & 3.551440 & 4.582538 & 3.695440 & 4.670595 & 3.754787 & 4.750471 & 3.791391 & 3.431442 & 2.668702\end{array}$ $\begin{array}{lllllllllllll}\text { BVAR2 } & 3.993817 & 3.217678 & 4.280075 & 3.456838 & 4.465277 & 3.605462 & 4.573579 & 3.673399 & 4.693650 & 3.732105 & 3.402612 & 2.662855\end{array}$ $\begin{array}{lllllllllllll}\text { BVAR3 } & 3.732416 & 2.983089 & 3.912973 & 3.151018 & 4.065521 & 3.272145 & 4.234938 & 3.387504 & 4.407741 & 3.508467 & 3.420873 & 2.767120\end{array}$

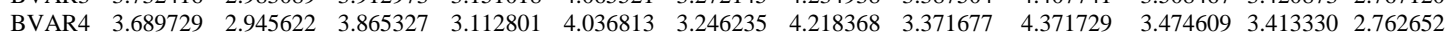

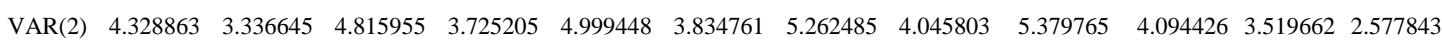
$\begin{array}{lllllllllllll}\text { BVAR1 } & 3.797618 & 2.998849 & 4.039335 & 3.195628 & 4.195887 & 3.321051 & 4.257734 & 3.369215 & 4.334430 & 3.416061 & 3.169352 & 2.431206\end{array}$

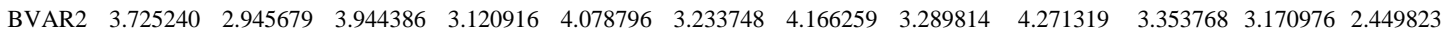
$\begin{array}{lllllllllllll}\text { BVAR3 } & 3.500115 & 2.729684 & 3.635803 & 2.859659 & 3.759752 & 2.967010 & 3.883878 & 3.057697 & 4.054369 & 3.182999 & 3.257645 & 2.612378\end{array}$

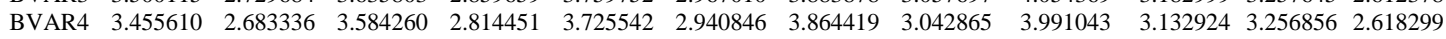

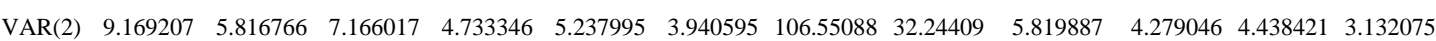

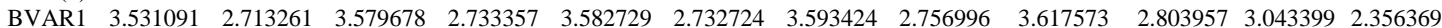

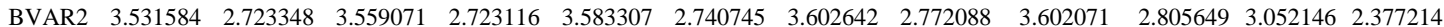
$\begin{array}{lllllllllllll}\text { BVAR3 } & 3.426493 & 2.622135 & 3.406866 & 2.584685 & 3.388663 & 2.574540 & 3.386178 & 2.585670 & 3.413973 & 2.632233 & 3.298014 & 2.677571\end{array}$

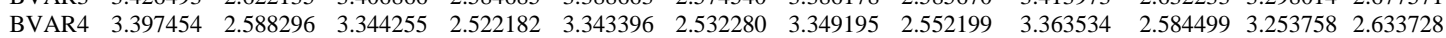

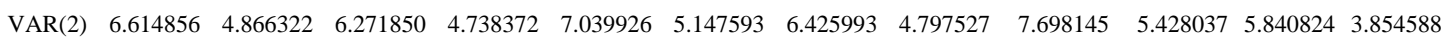
$\begin{array}{lllllllllllll}\text { BVAR1 } & 3.660079 & 2.839646 & 3.728276 & 2.877470 & 3.734515 & 2.882276 & 3.762497 & 2.922289 & 3.778606 & 2.958079 & 3.162561 & 2.463015\end{array}$

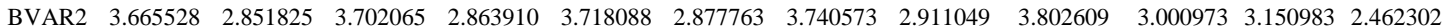
$\begin{array}{lllllllllllll}\text { BVAR3 } & 3.575071 & 2.774962 & 3.529572 & 2.715610 & 3.557259 & 2.739649 & 3.541282 & 2.739087 & 3.563884 & 2.779773 & 3.376575 & 2.747796\end{array}$ $\begin{array}{lllllllllllll}\text { BVAR4 } & 3.536856 & 2.734677 & 3.486953 & 2.671755 & 3.504762 & 2.693079 & 3.512819 & 2.713625 & 3.517804 & 2.732695 & 3.350006 & 2.712317\end{array}$

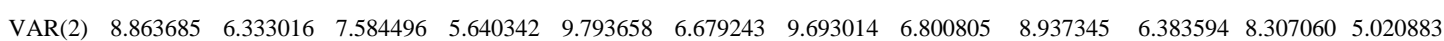
$\begin{array}{lllllllllllll}\text { BVAR1 } & 3.821654 & 2.994222 & 3.904125 & 3.046576 & 3.907610 & 3.047809 & 3.930620 & 3.078871 & 3.927038 & 3.102053 & 3.229146 & 2.513260\end{array}$

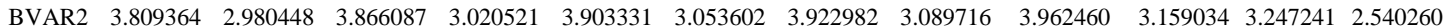
$\begin{array}{lllllllllllll}\text { BVAR3 } & 3.693154 & 2.895018 & 3.711670 & 2.898301 & 3.709158 & 2.897278 & 3.731214 & 2.929301 & 3.724193 & 2.929317 & 3.437700 & 2.796026\end{array}$ $\begin{array}{lllllllllllll}\text { BVAR4 } & 3.668832 & 2.872028 & 3.676103 & 2.862822 & 3.659952 & 2.850157 & 3.656811 & 2.853717 & 3.695755 & 2.907544 & 3.431987 & 2.780009\end{array}$

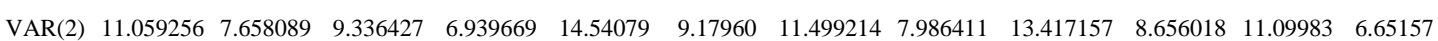
$\begin{array}{lllllllllllll}\text { BVAR1 } & 3.978572 & 3.139282 & 4.099155 & 3.229476 & 4.138013 & 3.262516 & 4.118019 & 3.258656 & 4.149411 & 3.312617 & 3.349929 & 2.612055\end{array}$ $\begin{array}{lllllllllllll}\text { BVAR2 } & 3.977398 & 3.144526 & 4.048213 & 3.190857 & 4.106580 & 3.247566 & 4.115803 & 3.273147 & 4.169012 & 3.352899 & 3.375640 & 2.646056\end{array}$ $\begin{array}{lllllllllllll}\text { BVAR3 } & 3.861636 & 3.064289 & 3.901387 & 3.088188 & 3.913092 & 3.099173 & 3.967308 & 3.153481 & 3.995572 & 3.193913 & 3.546365 & 2.875031\end{array}$ $\begin{array}{lllllllllllll}\text { BVAR4 } & 3.843570 & 3.044004 & 3.852881 & 3.039761 & 3.867478 & 3.048743 & 3.886660 & 3.077931 & 3.883450 & 3.093823 & 3.511981 & 2.842078\end{array}$

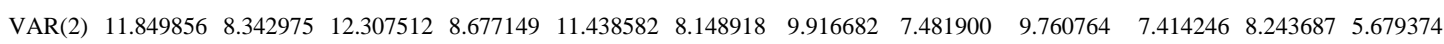
$\begin{array}{llllllllllllll}\text { BVAR1 } & 4.105596 & 3.249944 & 4.196453 & 3.315011 & 4.231578 & 3.352555 & 4.296974 & 3.432939 & 4.333651 & 3.492325 & 3.516985 & 2.757741\end{array}$ $\begin{array}{lllllllllllll}\text { BVAR2 } & 4.104060 & 3.262222 & 4.188436 & 3.318962 & 4.257999 & 3.391724 & 4.268755 & 3.424997 & 4.320151 & 3.505213 & 3.494499 & 2.743970\end{array}$ $\begin{array}{llllllllllllll}\text { BVAR3 } & 3.974140 & 3.170775 & 4.014777 & 3.194907 & 4.039272 & 3.218157 & 4.084954 & 3.267891 & 4.105856 & 3.298539 & 3.614303 & 2.925156\end{array}$ $\begin{array}{lllllllllllll}\text { BVAR4 } & 3.980389 & 3.173613 & 3.988237 & 3.161692 & 4.022405 & 3.203606 & 4.030510 & 3.216684 & 4.026813 & 3.228284 & 3.621642 & 2.931720\end{array}$ 


\section{Continued}

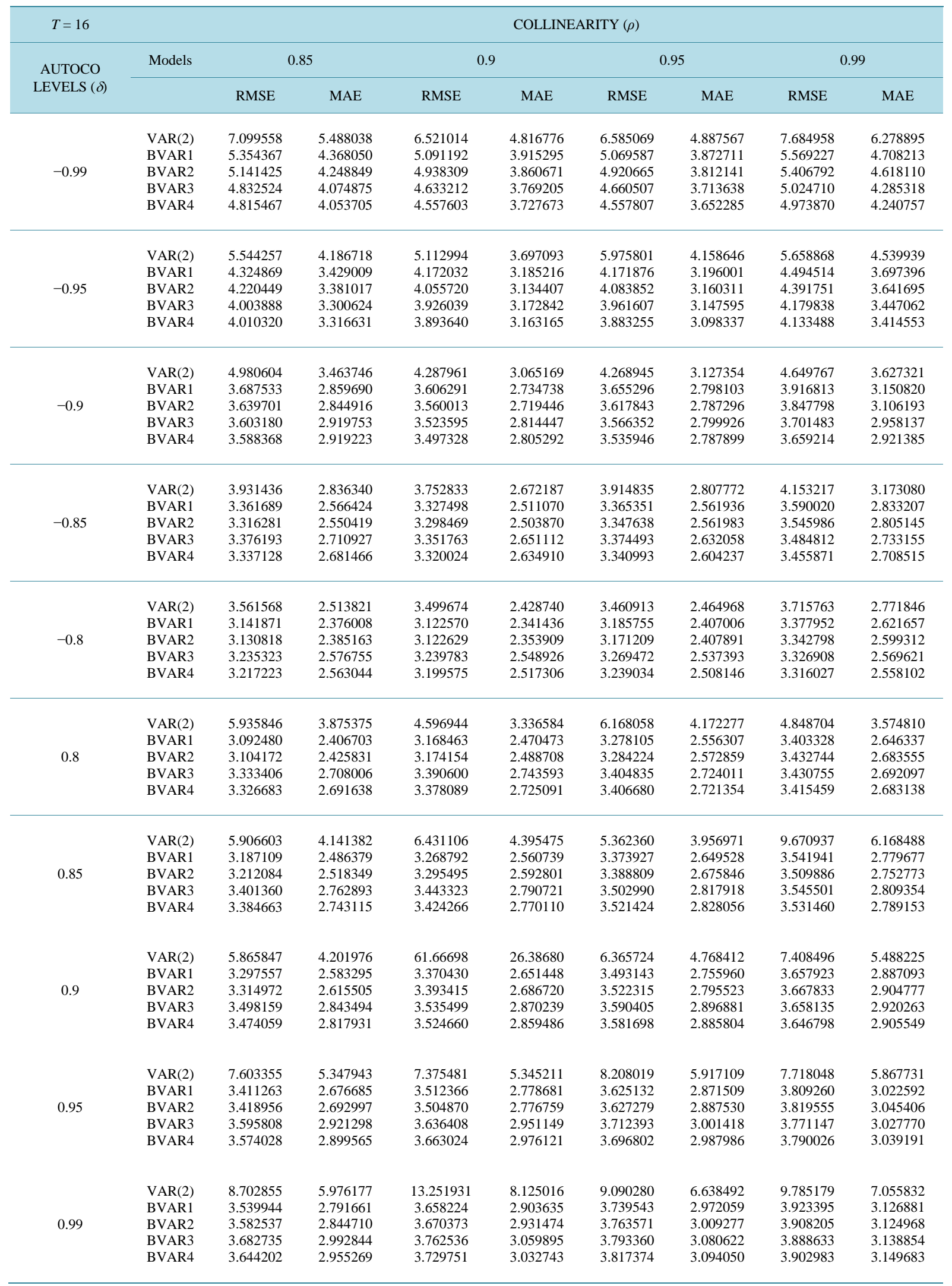


Table A3. The RMSE and MAE values of the model for $T=32$.

\begin{tabular}{|c|c|c|c|c|c|c|c|c|c|c|c|c|c|}
\hline \multirow{2}{*}{$\begin{array}{c}T=32 \\
\text { AUTOCO }\end{array}$} & & \multicolumn{12}{|c|}{ COLLINERITY $(\rho)$} \\
\hline & Models & \multicolumn{2}{|c|}{-0.99} & \multicolumn{2}{|c|}{-0.95} & \multicolumn{2}{|c|}{-0.9} & \multicolumn{2}{|c|}{-0.85} & \multicolumn{2}{|c|}{-0.8} & \multicolumn{2}{|c|}{0.8} \\
\hline (d) & & RMSE & MAE & RMSE & MAE & RMSE & MAE & RMSE & MAE & RMSE & MAE & RMSE & MAE \\
\hline
\end{tabular}

$\begin{array}{lllll}\text { VAR(2) } & 14.66326 & 12.02723 & 14.49286 & 12.43827\end{array}$ $\begin{array}{llllll}\text { BVAR1 } & 8.868865 & 7.985698 & 10.102584 & 8.909668\end{array}$ $\begin{array}{lllll}\text { BVAR2 } & 8.784330 & 7.892726 & 10.158533 & 8.929935\end{array}$ $\begin{array}{llllll}\text { BVAR3 } & 8.384759 & 7.436469 & 9.541767 & 8.280112\end{array}$ $\begin{array}{lllll}\text { BVAR4 } & 8.074158 & 7.105130 & 8.988559 & 7.738964\end{array}$

$\begin{array}{llllll}\text { VAR(2) } & 7.961468 & 6.722484 & 9.565847 & 7.915558\end{array}$ $\begin{array}{llllll}\text { BVAR1 } & 5.665201 & 4.906067 & 6.354424 & 5.432254\end{array}$ $\begin{array}{lllll}\text { BVAR2 } & 5.541683 & 4.792473 & 6.310088 & 5.371322\end{array}$ $\begin{array}{llllll}\text { BVAR3 } & 5.46905 & 4.72311 & 6.183366 & 5.277268\end{array}$ $\begin{array}{llllll}\text { BVAR4 } & 5.421003 & 4.674783 & 6.145703 & 5.225297\end{array}$

$\begin{array}{lllll}\text { VAR(2) } & 5.841933 & 4.849300 & 6.46564 & 5.31107\end{array}$ $\begin{array}{llllll}\text { BVAR1 } & 4.322259 & 3.621030 & 4.754169 & 3.949948\end{array}$ $\begin{array}{llllll}\text { BVAR2 } & 4.241650 & 3.547739 & 4.715024 & 3.908967\end{array}$ $\begin{array}{llllll}\text { BVAR3 } & 4.176008 & 3.489640 & 4.608181 & 3.837052\end{array}$ $\begin{array}{llllll}\text { BVAR4 } & 4.162391 & 3.486574 & 4.596080 & 3.822992\end{array}$

$\begin{array}{llllll}\text { VAR(2) } & 4.721373 & 3.824896 & 4.919008 & 4.011737\end{array}$ $\begin{array}{llllll}\text { BVAR1 } & 3.712822 & 3.034552 & 4.028760 & 3.276196\end{array}$ $\begin{array}{lllll}\text { BVAR2 } & 3.628486 & 2.960760 & 3.969323 & 3.220633\end{array}$ $\begin{array}{lllll}\text { BVAR3 } & 3.552807 & 2.890184 & 3.823768 & 3.111200\end{array}$ $\begin{array}{lllll}\text { BVAR4 } & 3.543409 & 2.891573 & 3.814055 & 3.105385\end{array}$

$\begin{array}{llllll}\text { VAR(2) } & 3.808556 & 3.052834 & 4.239043 & 3.368964\end{array}$ $\begin{array}{lllll}\text { BVAR1 } & 3.305102 & 2.644278 & 3.562016 & 2.845597\end{array}$ $\begin{array}{lllll}\text { BVAR1 } & 3.305102 & 2.644278 & 3.562016 & 2.845597 \\ \text { BVAR2 } & 3.243816 & 2.584761 & 3.528487 & 2.806951\end{array}$ $\begin{array}{lllll}\text { BVAR3 } & 3.186754 & 2.529159 & 3.380490 & 2.696674\end{array}$ $\begin{array}{lllll}\text { BVAR4 } & 3.150463 & 2.501813 & 3.365043 & 2.685716\end{array}$

$\begin{array}{llllll}\text { VAR(2) } & 3.732897 & 2.842723 & 3.49767 & 2.71882\end{array}$ $\begin{array}{lllll}\text { BVAR1 } & 3.313597 & 2.633425 & 3.316029 & 2.614770\end{array}$ $\begin{array}{lllll}\text { BVAR2 } & 3.266739 & 2.590845 & 3.283241 & 2.584817\end{array}$ $\begin{array}{lllll}\text { BVAR3 } & 3.087334 & 2.404135 & 3.037585 & 2.35304\end{array}$ $\begin{array}{lllll}\text { BVAR4 } & 3.023425 & 2.342413 & 2.993906 & 2.306430\end{array}$

$\begin{array}{llllll}\text { VAR(2) } & 3.986471 & 3.192565 & 4.249872 & 3.362915\end{array}$ $\begin{array}{llllll}\text { BVAR1 } & 3.607701 & 2.910104 & 3.625257 & 2.904919\end{array}$ $\begin{array}{lllll}\text { BVAR2 } & 3.562427 & 2.865036 & 3.575762 & 2.866121\end{array}$ $\begin{array}{lllll}\text { BVAR3 } & 3.333652 & 2.646939 & 3.324058 & 2.624462\end{array}$ $\begin{array}{lllll}\text { BVAR4 } & 3.293919 & 2.604514 & 3.248592 & 2.550524\end{array}$

$\begin{array}{lllll}\text { VAR(2) } & 7.341626 & 5.356272 & 5.689411 & 4.555991\end{array}$ $\begin{array}{lllll}\text { BVAR1 } & 4.019399 & 3.284759 & 4.059390 & 3.308286\end{array}$ $\begin{array}{lllll}\text { BVAR2 } & 3.995822 & 3.273663 & 4.016448 & 3.273467\end{array}$ $\begin{array}{lllll}\text { BVAR3 } & 3.759712 & 3.049572 & 3.733388 & 3.012368\end{array}$ $\begin{array}{lllll}\text { BVAR4 } & 3.683127 & 2.972334 & 3.683439 & 2.964628\end{array}$

$\begin{array}{llllll}\text { VAR(2) } & 8.145542 & 6.570007 & 9.508336 & 7.313701\end{array}$ $\begin{array}{lllll}\text { BVAR1 } & 4.641040 & 3.847504 & 4.723674 & 3.913560\end{array}$ $\begin{array}{lllll}\text { BVAR2 } & 4.607863 & 3.826239 & 4.687587 & 3.886086\end{array}$ $\begin{array}{llllll}\text { BVAR3 } & 4.354838 & 3.605449 & 4.375087 & 3.609372\end{array}$ $\begin{array}{lllll}\text { BVAR4 } & 4.251027 & 3.504735 & 4.311251 & 3.547510\end{array}$

$\begin{array}{lllll}\text { VAR(2) } & 9.910239 & 8.108691 & 10.231123 & 8.329465\end{array}$ BVAR1 $5.273782 \quad 4.418413 \quad 5.380396 \quad 4.505540$ $\begin{array}{lllll}\text { BVAR2 } & 5.263627 & 4.428455 & 5.375204 & 4.520336\end{array}$ $\begin{array}{lllll}\text { BVAR3 } & 5.008186 & 4.212243 & 5.127955 & 4.304945\end{array}$ $\begin{array}{lllll}\text { BVAR4 } & 5.015417 & 4.223160 & 5.058428 & 4.244019\end{array}$

$\begin{array}{llllllll}16.99112 & 14.07718 & 16.69649 & 13.97181 & 17.92657 & 14.74797 & 9.116383 & 7.499980\end{array}$ $\begin{array}{llllllll}11.231955 & 9.719722 & 11.92844 & 10.17850 & 12.40430 & 10.45598 & 6.826402 & 5.942840\end{array}$ $\begin{array}{llllllll}10.985347 & 9.457289 & 11.71362 & 9.93686 & 11.95375 & 10.00748 & 6.760333 & 5.873528\end{array}$ $\begin{array}{lllllllll}10.144903 & 8.641844 & 10.671738 & 8.963683 & 11.066390 & 9.201769 & 6.793669 & 5.796042\end{array}$ $\begin{array}{lllllllll}9.631319 & 8.138354 & 9.936045 & 8.274346 & 10.374309 & 8.564269 & 6.590898 & 5.585019\end{array}$

$\begin{array}{llllllll}10.085588 & 8.349892 & 10.48444 & 8.62861 & 10.641119 & 8.743647 & 5.733142 & 4.571978\end{array}$ $\begin{array}{lllllllll}6.891470 & 5.789703 & 7.365829 & 6.097293 & 7.715081 & 6.318872 & 4.471984 & 3.718808\end{array}$ $\begin{array}{lllllllll}6.866607 & 5.750905 & 7.319716 & 6.049675 & 7.653206 & 6.261866 & 4.451677 & 3.698245\end{array}$ $\begin{array}{lllllllll}6.669163 & 5.596393 & 7.157648 & 5.938573 & 7.464696 & 6.126180 & 4.725151 & 3.960498\end{array}$ $\begin{array}{lllllllll}6.712264 & 5.627363 & 7.065304 & 5.851712 & 7.335338 & 6.017297 & 4.644578 & 3.891115\end{array}$

$\begin{array}{llllllll}7.004660 & 5.702322 & 7.361425 & 5.974679 & 7.928795 & 6.366810 & 4.045249 & 3.175013\end{array}$ $\begin{array}{lllllllll}5.150787 & 4.227924 & 5.500133 & 4.458332 & 5.802533 & 4.658706 & 3.578792 & 2.909100\end{array}$ $\begin{array}{lllllllll}5.130988 & 4.197488 & 5.450288 & 4.405916 & 5.746614 & 4.603175 & 3.536624 & 2.878184\end{array}$ $\begin{array}{lllllllll}4.987416 & 4.102516 & 5.294466 & 4.306556 & 5.550953 & 4.473654 & 3.858831 & 3.225961\end{array}$ $\begin{array}{lllllllll}4.983092 & 4.096438 & 5.279011 & 4.294784 & 5.533153 & 4.462557 & 3.833990 & 3.203973\end{array}$

$\begin{array}{llllllll}5.380163 & 4.337040 & 5.722676 & 4.590195 & 5.810400 & 4.656082 & 3.366717 & 2.590805\end{array}$ $\begin{array}{lllllllll}4.305397 & 3.472099 & 4.600571 & 3.672320 & 4.885385 & 3.869882 & 3.132456 & 2.511884\end{array}$ $\begin{array}{lllllllll}4.293373 & 3.452355 & 4.606864 & 3.673849 & 4.845367 & 3.836360 & 3.095521 & 2.490810\end{array}$ $\begin{array}{lllllllll}4.136698 & 3.342128 & 4.384609 & 3.510920 & 4.621882 & 3.663847 & 3.464844 & 2.886642\end{array}$ $\begin{array}{llllllll}3.331461 & 4.399845 & 3.526143 & 4.616055 & 3.664718 & 3.408097 & 2.840940\end{array}$

$\begin{array}{llllllll}4.291196 & 3.438790 & 4.549485 & 3.624453 & 5.149671 & 3.989592 & 2.831364 & 2.138279\end{array}$ $\begin{array}{lllllllll}3.823200 & 3.035168 & 4.069328 & 3.210558 & 4.345022 & 3.415132 & 2.864495 & 2.272026\end{array}$ $\begin{array}{lllllllll}3.812184 & 3.021101 & 4.060453 & 3.201939 & 4.315298 & 3.390845 & 2.856141 & 2.274289\end{array}$ $\begin{array}{llllllll}3.632793 & 2.886910 & 3.869228 & 3.052689 & 4.069984 & 3.189529 & 3.217359 & 2.665676\end{array}$ $\begin{array}{llllllll}3.625036 & 2.881452 & 3.831084 & 3.025776 & 4.016312 & 3.149059 & 3.152122 & 2.608140\end{array}$

$\begin{array}{llllllll}3.530011 & 2.742267 & 3.443827 & 2.693251 & 3.734037 & 2.855492 & 2.715320 & 2.054366\end{array}$ $\begin{array}{lllllllll}3.353025 & 2.652087 & 3.380967 & 2.684577 & 3.465971 & 2.777635 & 2.984894 & 2.399354\end{array}$ $\begin{array}{llllllll}3.298040 & 2.598611 & 3.364418 & 2.672576 & 3.443605 & 2.759310 & 2.987995 & 2.407203\end{array}$ $\begin{array}{llllllll}3.043436 & 2.357823 & 3.066925 & 2.389038 & 3.092260 & 2.417901 & 3.089470 & 2.515941\end{array}$ $\begin{array}{llllllll}2.995605 & 2.311568 & 3.017887 & 2.340079 & 3.023834 & 2.353389 & 2.949539 & 2.376680\end{array}$

$\begin{array}{llllllll}4.253023 & 3.344118 & 4.463272 & 3.502106 & 4.314869 & 3.431765 & 3.159290 & 2.420794\end{array}$ $\begin{array}{llllllll}3.638141 & 2.918589 & 3.698064 & 2.978903 & 3.742047 & 3.034855 & 3.172923 & 2.562054\end{array}$ $\begin{array}{llllllll}3.595043 & 2.876212 & 3.630191 & 2.922750 & 3.726171 & 3.022565 & 3.192029 & 2.582575\end{array}$ $\begin{array}{llllllll}3.330410 & 2.631034 & 3.326336 & 2.630352 & 3.344339 & 2.654069 & 3.25450 & 2.65923\end{array}$ $\begin{array}{llllllll}3.253474 & 2.559676 & 3.294338 & 2.600510 & 3.273909 & 2.589784 & 3.141835 & 2.544247\end{array}$

5.555286 4.078738 4.060717 3.742516 3.674642 $\begin{array}{lllllll}4.492584 & 5.899174 & 4.724377 & 5.607266 & 4.499744 & 4.056663 & 3.135429\end{array}$ $\begin{array}{llllllll}3.327450 & 4.120367 & 3.374136 & 4.173862 & 3.428979 & 3.484817 & 2.824452\end{array}$ $\begin{array}{llllllll}3.312161 & 4.089725 & 3.347477 & 4.111696 & 3.376803 & 3.497303 & 2.838614\end{array}$ $\begin{array}{llllllll}3.023017 & 3.750787 & 3.028037 & 3.756089 & 3.037540 & 3.504424 & 2.871266\end{array}$ $\begin{array}{lllllll}2.956385 & 3.685515 & 2.964958 & 3.690723 & 2.976093 & 3.415987 & 2.782976\end{array}$
9.459770 4.778199 4.721712 4.448615 4.337292 $\begin{array}{llllllll}7.365879 & 8.430181 & 6.769306 & 8.532818 & 6.899061 & 6.454197 & 4.744584\end{array}$ $\begin{array}{llllllll}3.961433 & 4.808107 & 3.997918 & 4.862071 & 4.066127 & 3.910754 & 3.172627\end{array}$ $\begin{array}{llllllll}3.922829 & 4.781962 & 3.987344 & 4.807260 & 4.024241 & 3.920832 & 3.188766\end{array}$ $\begin{array}{llllllll}3.677085 & 4.379007 & 3.607476 & 4.466676 & 3.694930 & 3.905550 & 3.204876\end{array}$ $\begin{array}{lllllll}3.572457 & 4.373170 & 3.603382 & 4.444653 & 3.672500 & 3.816804 & 3.122586\end{array}$ $\begin{array}{llllllll}12.840126 & 9.674357 & 11.277482 & 8.859553 & 11.194561 & 8.953436 & 8.788139 & 6.385497\end{array}$ $\begin{array}{lllllllll}5.444321 & 4.564565 & 5.516622 & 4.648955 & 5.600328 & 4.735702 & 4.428247 & 3.595652\end{array}$ $\begin{array}{lllllllll}5.469501 & 4.609911 & 5.551135 & 4.699438 & 5.608524 & 4.757579 & 4.475890 & 3.659284\end{array}$ $\begin{array}{lllllllll}5.109050 & 4.278658 & 5.172752 & 4.340818 & 5.181618 & 4.347265 & 4.395432 & 3.616917\end{array}$ $\begin{array}{lllllllll}5.084977 & 4.264155 & 5.202984 & 4.367991 & 5.241316 & 4.412264 & 4.337231 & 3.569237\end{array}$ 


\section{Continued}

\begin{tabular}{|c|c|c|c|c|c|c|c|c|c|}
\hline \multirow{3}{*}{$\begin{array}{c}\text { AUTOCO } \\
\text { LEVELS }(\delta)\end{array}$} & \multirow{3}{*}{ Models } & \multicolumn{8}{|c|}{ COLLINEARITY $(\rho)$} \\
\hline & & \multicolumn{2}{|c|}{0.85} & \multicolumn{2}{|c|}{0.9} & \multicolumn{2}{|c|}{0.95} & \multicolumn{2}{|c|}{0.99} \\
\hline & & RMSE & MAE & RMSE & MAE & RMSE & MAE & RMSE & MAE \\
\hline-0.99 & $\begin{array}{l}\text { VAR(2) } \\
\text { BVAR1 } \\
\text { BVAR2 } \\
\text { BVAR3 } \\
\text { BVAR4 }\end{array}$ & $\begin{array}{l}8.183544 \\
6.255208 \\
6.225226 \\
6.319025 \\
6.127081\end{array}$ & $\begin{array}{l}6.409968 \\
5.232098 \\
5.205574 \\
5.269976 \\
5.083030\end{array}$ & $\begin{array}{l}8.171164 \\
5.954346 \\
5.883918 \\
5.972126 \\
5.832870\end{array}$ & $\begin{array}{l}5.886140 \\
4.687005 \\
4.642069 \\
4.828020 \\
4.702794\end{array}$ & $\begin{array}{c}13.761278 \\
6.061907 \\
5.971372 \\
5.912063 \\
5.782263\end{array}$ & $\begin{array}{l}8.646975 \\
4.846714 \\
4.783738 \\
4.753435 \\
4.637809\end{array}$ & $\begin{array}{l}9.660148 \\
6.838000 \\
6.707916 \\
6.602793 \\
6.419753\end{array}$ & $\begin{array}{l}8.225798 \\
6.038562 \\
5.924713 \\
5.748045 \\
5.570510\end{array}$ \\
\hline-0.95 & $\begin{array}{l}\text { VAR(2) } \\
\text { BVAR1 } \\
\text { BVAR2 } \\
\text { BVAR3 } \\
\text { BVAR4 }\end{array}$ & $\begin{array}{l}5.419512 \\
4.278932 \\
4.210253 \\
4.467069 \\
4.400286\end{array}$ & $\begin{array}{l}4.117640 \\
3.470092 \\
3.421989 \\
3.733129 \\
3.670985\end{array}$ & $\begin{array}{l}5.410199 \\
4.144168 \\
4.077051 \\
4.256684 \\
4.199415\end{array}$ & $\begin{array}{l}3.927946 \\
3.266110 \\
3.225904 \\
3.515731 \\
3.458578\end{array}$ & $\begin{array}{l}5.650153 \\
4.191661 \\
4.094175 \\
4.196531 \\
4.165205\end{array}$ & $\begin{array}{l}4.293156 \\
3.340760 \\
3.269946 \\
3.405027 \\
3.382798\end{array}$ & $\begin{array}{l}6.297137 \\
4.561301 \\
4.505046 \\
4.544496 \\
4.442278\end{array}$ & $\begin{array}{l}5.262456 \\
3.850747 \\
3.817464 \\
3.832547 \\
3.746108\end{array}$ \\
\hline-0.9 & $\begin{array}{l}\text { VAR(2) } \\
\text { BVAR1 } \\
\text { BVAR2 } \\
\text { BVAR3 } \\
\text { BVAR4 }\end{array}$ & $\begin{array}{l}4.156610 \\
3.435454 \\
3.399575 \\
3.682001 \\
3.621324\end{array}$ & $\begin{array}{l}3.094199 \\
2.741574 \\
2.721451 \\
3.079270 \\
3.027212\end{array}$ & $\begin{array}{l}3.896820 \\
3.401299 \\
3.320739 \\
3.530001 \\
3.467940\end{array}$ & $\begin{array}{l}2.847562 \\
2.662821 \\
2.612250 \\
2.911328 \\
2.857803\end{array}$ & $\begin{array}{l}4.261132 \\
3.458562 \\
3.366029 \\
3.458037 \\
3.393824\end{array}$ & $\begin{array}{l}3.219722 \\
2.727781 \\
2.656156 \\
2.778508 \\
2.726651\end{array}$ & $\begin{array}{l}4.677126 \\
3.701453 \\
3.609712 \\
3.661370 \\
3.570615\end{array}$ & $\begin{array}{l}3.795311 \\
3.041555 \\
2.961387 \\
2.989493 \\
2.915543\end{array}$ \\
\hline-0.85 & $\begin{array}{l}\text { VAR(2) } \\
\text { BVAR1 } \\
\text { BVAR2 } \\
\text { BVAR3 } \\
\text { BVAR4 }\end{array}$ & $\begin{array}{l}3.135666 \\
3.062404 \\
3.017451 \\
3.306289 \\
3.241433\end{array}$ & $\begin{array}{l}2.338310 \\
2.414592 \\
2.389854 \\
2.750929 \\
2.694858\end{array}$ & $\begin{array}{l}3.172442 \\
3.010045 \\
2.962397 \\
3.172644 \\
3.096611\end{array}$ & $\begin{array}{l}2.309062 \\
2.341620 \\
2.308213 \\
2.599510 \\
2.534366\end{array}$ & $\begin{array}{l}3.577560 \\
3.091658 \\
3.001191 \\
3.127430 \\
3.030196\end{array}$ & $\begin{array}{l}2.635234 \\
2.417700 \\
2.337642 \\
2.485342 \\
2.404734\end{array}$ & $\begin{array}{l}3.660426 \\
3.258536 \\
3.174013 \\
3.231151 \\
3.153569\end{array}$ & $\begin{array}{l}2.926463 \\
2.618544 \\
2.543425 \\
2.572105 \\
2.509348\end{array}$ \\
\hline-0.8 & $\begin{array}{l}\text { VAR(2) } \\
\text { BVAR1 } \\
\text { BVAR2 } \\
\text { BVAR3 } \\
\text { BVAR4 }\end{array}$ & $\begin{array}{l}2.784587 \\
2.814113 \\
2.765962 \\
3.085280 \\
3.020429\end{array}$ & $\begin{array}{l}2.049203 \\
2.201595 \\
2.171642 \\
2.549175 \\
2.492657\end{array}$ & $\begin{array}{l}2.813672 \\
2.794123 \\
2.746840 \\
2.967020 \\
2.893054\end{array}$ & $\begin{array}{l}2.019906 \\
2.158500 \\
2.124867 \\
2.412485 \\
2.342757\end{array}$ & $\begin{array}{l}2.869672 \\
2.852458 \\
2.776470 \\
2.916558 \\
2.828328\end{array}$ & $\begin{array}{l}2.139474 \\
2.207132 \\
2.142921 \\
2.295532 \\
2.216337\end{array}$ & $\begin{array}{l}3.106624 \\
2.995363 \\
2.918323 \\
2.985039 \\
2.901840\end{array}$ & $\begin{array}{l}2.427903 \\
2.368584 \\
2.295031 \\
2.333521 \\
2.259205\end{array}$ \\
\hline 0.8 & $\begin{array}{l}\text { VAR(2) } \\
\text { BVAR1 } \\
\text { BVAR2 } \\
\text { BVAR3 } \\
\text { BVAR4 }\end{array}$ & $\begin{array}{l}2.842527 \\
3.025476 \\
3.029957 \\
3.132378 \\
2.999509\end{array}$ & $\begin{array}{l}2.152664 \\
2.440972 \\
2.451758 \\
2.549708 \\
2.414277\end{array}$ & $\begin{array}{l}2.810912 \\
3.090351 \\
3.089930 \\
3.178715 \\
3.056396\end{array}$ & $\begin{array}{l}2.169077 \\
2.500016 \\
2.505098 \\
2.583167 \\
2.455273\end{array}$ & $\begin{array}{l}2.952656 \\
3.150829 \\
3.150130 \\
3.216895 \\
3.107721\end{array}$ & $\begin{array}{l}2.290625 \\
2.548073 \\
2.554562 \\
2.601105 \\
2.487607\end{array}$ & $\begin{array}{l}3.307669 \\
3.246468 \\
3.226010 \\
3.200862 \\
3.133152\end{array}$ & $\begin{array}{l}2.547600 \\
2.615978 \\
2.599928 \\
2.555826 \\
2.484048\end{array}$ \\
\hline 0.85 & $\begin{array}{l}\text { VAR(2) } \\
\text { BVAR1 } \\
\text { BVAR2 } \\
\text { BVAR3 } \\
\text { BVAR4 }\end{array}$ & $\begin{array}{l}3.331595 \\
3.252981 \\
3.249162 \\
3.304187 \\
3.196640\end{array}$ & $\begin{array}{l}2.580975 \\
2.642329 \\
2.644656 \\
2.702467 \\
2.590177\end{array}$ & $\begin{array}{l}3.793309 \\
3.321229 \\
3.314847 \\
3.351939 \\
3.247441\end{array}$ & $\begin{array}{l}2.842995 \\
2.710138 \\
2.705682 \\
2.740538 \\
2.631263\end{array}$ & $\begin{array}{c}3.717028 \\
3.38536 \\
3.408850 \\
3.403712 \\
3.310554\end{array}$ & $\begin{array}{c}2.929144 \\
2.76281 \\
2.789758 \\
2.774270 \\
2.676317\end{array}$ & $\begin{array}{l}3.807675 \\
3.508926 \\
3.490607 \\
3.407476 \\
3.338701\end{array}$ & $\begin{array}{l}3.040937 \\
2.858963 \\
2.846291 \\
2.748869 \\
2.681568\end{array}$ \\
\hline 0.9 & $\begin{array}{l}\text { VAR(2) } \\
\text { BVAR1 } \\
\text { BVAR2 } \\
\text { BVAR3 } \\
\text { BVAR4 }\end{array}$ & $\begin{array}{l}4.293119 \\
3.572668 \\
3.556106 \\
3.563569 \\
3.450073\end{array}$ & $\begin{array}{l}3.336612 \\
2.915571 \\
2.910716 \\
2.925840 \\
2.813604\end{array}$ & $\begin{array}{l}4.519025 \\
3.636147 \\
3.615754 \\
3.606210 \\
3.531307\end{array}$ & $\begin{array}{l}3.574176 \\
2.983366 \\
2.975477 \\
2.962859 \\
2.885237\end{array}$ & $\begin{array}{l}4.776709 \\
3.758688 \\
3.723412 \\
3.672251 \\
3.610613\end{array}$ & $\begin{array}{l}3.802752 \\
3.096874 \\
3.070471 \\
3.014741 \\
2.949436\end{array}$ & $\begin{array}{l}5.144674 \\
3.870640 \\
3.847066 \\
3.737134 \\
3.687554\end{array}$ & $\begin{array}{l}4.141709 \\
3.185365 \\
3.171730 \\
3.062082 \\
3.008539\end{array}$ \\
\hline 0.95 & $\begin{array}{l}\text { VAR(2) } \\
\text { BVAR1 } \\
\text { BVAR2 } \\
\text { BVAR3 } \\
\text { BVAR4 }\end{array}$ & $\begin{array}{l}5.994791 \\
3.986404 \\
3.967685 \\
3.945150 \\
3.856760\end{array}$ & $\begin{array}{l}4.662920 \\
3.262290 \\
3.252945 \\
3.251867 \\
3.163625\end{array}$ & $\begin{array}{l}6.393341 \\
4.055031 \\
4.054657 \\
4.019074 \\
3.967639\end{array}$ & $\begin{array}{l}5.021365 \\
3.335684 \\
3.352590 \\
3.325958 \\
3.273244\end{array}$ & $\begin{array}{l}6.471447 \\
4.227944 \\
4.208908 \\
4.100253 \\
4.062039\end{array}$ & $\begin{array}{l}5.281929 \\
3.503378 \\
3.497806 \\
3.403174 \\
3.362454\end{array}$ & $\begin{array}{l}7.911398 \\
4.403969 \\
4.414250 \\
4.217873 \\
4.158784\end{array}$ & $\begin{array}{l}6.255814 \\
3.662445 \\
3.682337 \\
3.500569 \\
3.443901\end{array}$ \\
\hline 0.99 & $\begin{array}{l}\text { VAR(2) } \\
\text { BVAR1 } \\
\text { BVAR2 } \\
\text { BVAR3 } \\
\text { BVAR4 }\end{array}$ & $\begin{array}{c}7.98958 \\
4.505180 \\
4.503203 \\
4.491434 \\
4.443043\end{array}$ & $\begin{array}{c}6.14510 \\
3.695378 \\
3.711759 \\
3.720080 \\
3.678880\end{array}$ & $\begin{array}{l}8.463565 \\
4.632210 \\
4.631356 \\
4.494059 \\
4.505566\end{array}$ & $\begin{array}{l}6.573965 \\
3.841432 \\
3.848661 \\
3.736156 \\
3.751414\end{array}$ & $\begin{array}{c}10.269673 \\
4.758618 \\
4.781808 \\
4.678435 \\
4.594163\end{array}$ & $\begin{array}{l}7.868398 \\
3.972241 \\
4.003160 \\
3.922521 \\
3.840406\end{array}$ & $\begin{array}{c}10.538601 \\
4.987143 \\
4.985718 \\
4.869713 \\
4.812612\end{array}$ & $\begin{array}{l}8.359982 \\
4.177887 \\
4.189164 \\
4.101869 \\
4.041900\end{array}$ \\
\hline
\end{tabular}


Table A4. The RMSE and MAE values of the model for $T=64$.

\begin{tabular}{|c|c|c|c|c|c|c|c|c|c|c|c|c|c|}
\hline \multicolumn{2}{|l|}{$T=64$} & \multicolumn{12}{|c|}{ COLLINERITY $(\rho)$} \\
\hline \multirow{2}{*}{$\begin{array}{l}\text { AUTOCO } \\
\text { LEVELS }(\delta)\end{array}$} & \multirow[t]{2}{*}{ Models } & \multicolumn{2}{|c|}{-0.99} & \multicolumn{2}{|c|}{-0.95} & \multicolumn{2}{|c|}{-0.9} & \multicolumn{2}{|c|}{-0.85} & \multicolumn{2}{|c|}{-0.8} & \multicolumn{2}{|c|}{0.8} \\
\hline & & RMSE & MAE & RMSE & MAE & RMSE & MAE & RMSE & MAE & RMSE & MAE & RMSE & MAE \\
\hline
\end{tabular}

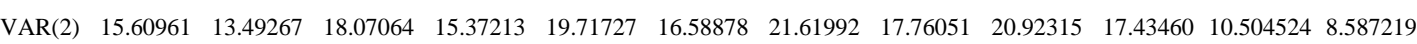
$\begin{array}{lllllllllllll}\text { BVAR1 } & 11.46939 & 10.19435 & 13.09585 & 11.39752 & 14.29593 & 12.23065 & 14.99266 & 12.65584 & 15.31937 & 12.80392 & 8.504290 & 7.257119\end{array}$ $\begin{array}{lllllllllllll}\text { BVAR2 } & 11.33424 & 10.04413 & 12.83739 & 11.13458 & 13.95068 & 11.87831 & 14.40817 & 12.10325 & 14.71918 & 12.21020 & 8.433383 & 7.186270\end{array}$ $\begin{array}{lllllllllllll}\text { BVAR3 } & 10.393633 & 9.075173 & 11.674118 & 9.980774 & 12.70827 & 10.70607 & 13.29599 & 11.07513 & 13.74958 & 11.36994 & 8.130523 & 6.846395\end{array}$

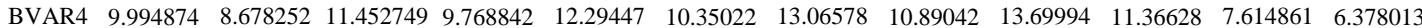

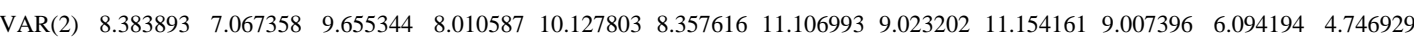

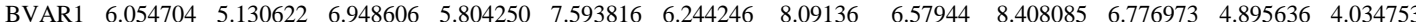
$\begin{array}{lllllllllllll}\text { BVAR2 } & 6.028815 & 5.111177 & 6.880661 & 5.732709 & 7.601027 & 6.241448 & 8.033711 & 6.537276 & 8.406621 & 6.784209 & 4.828257 & 3.977608\end{array}$ $\begin{array}{llllllllllllll}\text { BVAR3 } & 6.101289 & 5.177180 & 6.962924 & 5.820154 & 7.598739 & 6.263761 & 8.115210 & 6.630601 & 8.386429 & 6.791154 & 5.079400 & 4.240575\end{array}$

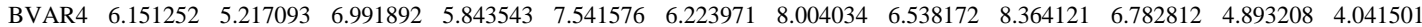

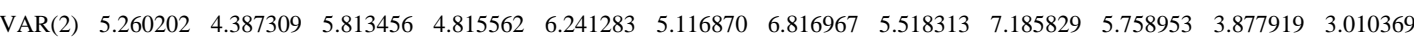
$\begin{array}{lllllllllllll}\text { BVAR1 } & 4.310264 & 3.560062 & 4.871217 & 3.976989 & 5.326900 & 4.285672 & 5.673882 & 4.512546 & 6.033064 & 4.754550 & 3.733896 & 3.085129\end{array}$ $\begin{array}{lllllllllllll}\text { BVAR2 } & 4.278509 & 3.540720 & 4.852454 & 3.959338 & 5.315123 & 4.274960 & 5.703989 & 4.540852 & 5.966621 & 4.709866 & 3.678327 & 3.034653\end{array}$ $\begin{array}{lllllllllllll}\text { BVAR3 } & 4.281429 & 3.538884 & 4.860214 & 3.975913 & 5.317118 & 4.297216 & 5.682409 & 4.540495 & 5.892455 & 4.666515 & 4.038027 & 3.412802\end{array}$

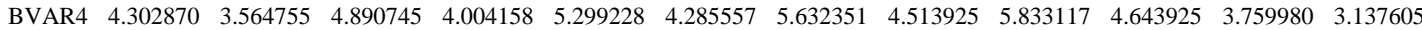

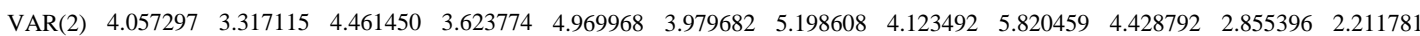
$\begin{array}{lllllllllllll}\text { BVAR1 } & 3.498572 & 2.835034 & 3.897501 & 3.136541 & 4.308327 & 3.425581 & 4.627766 & 3.638906 & 4.907163 & 3.824280 & 3.223494 & 2.661245\end{array}$ $\begin{array}{lllllllllllll}\text { BVAR2 } & 3.469369 & 2.814946 & 3.931286 & 3.160528 & 4.323880 & 3.432997 & 4.606818 & 3.621454 & 4.871123 & 3.800238 & 3.165957 & 2.610390\end{array}$ $\begin{array}{lllllllllllll}\text { BVAR3 } & 3.489324 & 2.835115 & 3.905329 & 3.150414 & 4.257981 & 3.392493 & 4.555086 & 3.597710 & 4.795215 & 3.751707 & 3.580932 & 3.034515\end{array}$

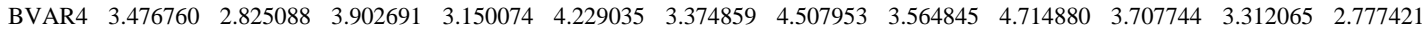

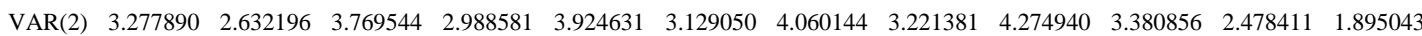
$\begin{array}{lllllllllllll}\text { BVAR1 } & 3.031720 & 2.415329 & 3.373929 & 2.681704 & 3.716239 & 2.927085 & 4.019306 & 3.140814 & 4.284086 & 3.320737 & 2.922556 & 2.404180\end{array}$ $\begin{array}{lllllllllllll}\text { BVAR2 } & 2.999372 & 2.389678 & 3.391303 & 2.692547 & 3.734031 & 2.940980 & 4.011632 & 3.131288 & 4.238038 & 3.288068 & 2.866138 & 2.354839\end{array}$ $\begin{array}{llllllllllllll}\text { BVAR3 } & 3.029278 & 2.422018 & 3.342599 & 2.662681 & 3.650452 & 2.883028 & 3.910502 & 3.060122 & 4.107552 & 3.188801 & 3.321659 & 2.806316\end{array}$ $\begin{array}{llllllllllllll}\text { BVAR4 } & 3.001746 & 2.399163 & 3.334189 & 2.658396 & 3.625195 & 2.863128 & 3.854035 & 3.023937 & 4.030069 & 3.141156 & 3.041197 & 2.549028\end{array}$

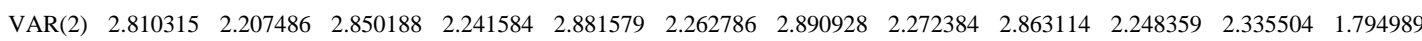

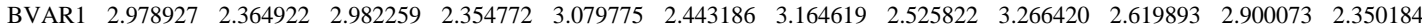
$\begin{array}{lllllllllllll}\text { BVAR2 } & 2.942782 & 2.329299 & 2.946586 & 2.323064 & 3.032298 & 2.399321 & 3.122161 & 2.483368 & 3.210009 & 2.567070 & 2.806345 & 2.259498\end{array}$ $\begin{array}{lllllllllllll}\text { BVAR3 } & 2.807824 & 2.201907 & 2.782325 & 2.176855 & 2.818668 & 2.211739 & 2.849104 & 2.237374 & 2.882040 & 2.268192 & 2.804422 & 2.269090\end{array}$ $\begin{array}{lllllllllllll}\text { BVAR4 } & 2.765080 & 2.166512 & 2.767764 & 2.161917 & 2.786543 & 2.181607 & 2.817992 & 2.208545 & 2.833942 & 2.226876 & 2.658767 & 2.130071\end{array}$

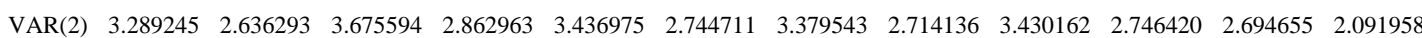

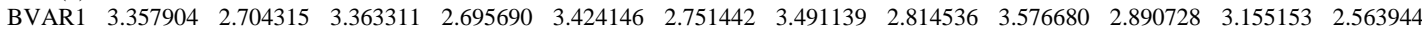

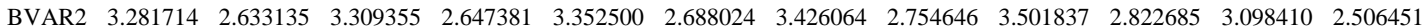
$\begin{array}{lllllllllllll}\text { BVAR3 } & 3.122439 & 2.487813 & 3.096772 & 2.461153 & 3.125829 & 2.485300 & 3.152716 & 2.507731 & 3.167574 & 2.519834 & 3.029096 & 2.458432\end{array}$ $\begin{array}{lllllllllllll}\text { BVAR4 } & 3.055098 & 2.426874 & 3.076407 & 2.437918 & 3.096576 & 2.459122 & 3.108977 & 2.469156 & 3.130619 & 2.489803 & 2.892868 & 2.330261\end{array}$

$\begin{array}{lllllllllllll}\text { VAR(2) } & 4.303766 & 3.541903 & 4.534682 & 3.732747 & 4.569826 & 3.728954 & 4.579045 & 3.717489 & 4.696541 & 3.817790 & 3.358154 & 2.630087\end{array}$

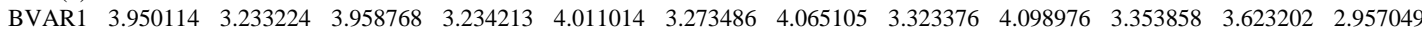

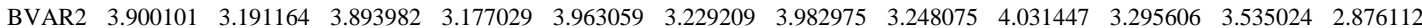
$\begin{array}{llllllllllllll}\text { BVAR3 } & 3.645300 & 2.953421 & 3.643486 & 2.949505 & 3.660824 & 2.960681 & 3.699359 & 2.994209 & 3.700648 & 2.997655 & 3.403221 & 2.775237\end{array}$ $\begin{array}{lllllllllllll}\text { BVAR4 } & 3.59670 & 2.91165 & 3.614176 & 2.923150 & 3.619210 & 2.925299 & 3.640562 & 2.939275 & 3.674514 & 2.973086 & 3.282248 & 2.662536\end{array}$

$\begin{array}{lllllllllllll}\text { VAR(2) } & 7.399854 & 6.076538 & 7.475618 & 6.243605 & 7.656434 & 6.345574 & 7.420693 & 6.142446 & 7.710607 & 6.315597 & 8.534871 & 5.660741\end{array}$ $\begin{array}{llllllllllllll}\text { BVAR1 } & 5.137436 & 4.298933 & 5.218394 & 4.359780 & 5.265360 & 4.397508 & 5.243674 & 4.381406 & 5.342945 & 4.469960 & 4.439834 & 3.635345\end{array}$ $\begin{array}{lllllllllllll}\text { BVAR2 } & 5.085147 & 4.255915 & 5.110901 & 4.267637 & 5.210220 & 4.357695 & 5.194227 & 4.340276 & 5.233543 & 4.372968 & 4.382403 & 3.585276\end{array}$ $\begin{array}{lllllllllllll}\text { BVAR3 } & 4.763168 & 3.962527 & 4.799530 & 3.980349 & 4.826999 & 4.004062 & 4.895486 & 4.065861 & 4.905022 & 4.075951 & 4.199595 & 3.450845\end{array}$ $\begin{array}{lllllllllllll}\text { BVAR4 } & 4.705414 & 3.908203 & 4.795625 & 3.984978 & 4.792639 & 3.975185 & 4.818079 & 3.992608 & 4.819966 & 3.996624 & 4.125768 & 3.385711\end{array}$

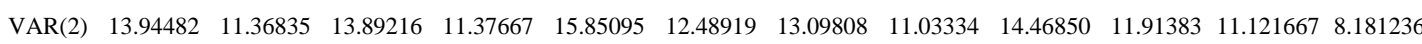
$\begin{array}{lllllllllllll}\text { BVAR1 } & 7.080895 & 6.054866 & 7.306613 & 6.244493 & 7.432235 & 6.351495 & 7.486951 & 6.404039 & 7.545406 & 6.459999 & 5.855950 & 4.799674\end{array}$ $\begin{array}{lllllllllllll}\text { BVAR2 } & 7.054366 & 6.043512 & 7.290005 & 6.245802 & 7.352421 & 6.297172 & 7.475933 & 6.413686 & 7.590449 & 6.522315 & 5.868612 & 4.826717\end{array}$ $\begin{array}{lllllllllllll}\text { BVAR3 } & 6.834535 & 5.857399 & 7.001530 & 5.976842 & 6.969472 & 5.943162 & 7.058195 & 6.022529 & 7.348942 & 6.290457 & 5.691735 & 4.698703\end{array}$ $\begin{array}{lllllllllllll}\text { BVAR4 } & 6.828429 & 5.847847 & 6.924037 & 5.910194 & 7.111204 & 6.079811 & 7.167811 & 6.121913 & 7.229497 & 6.174797 & 5.638314 & 4.654588\end{array}$ 


\section{Continued}

\begin{tabular}{|c|c|c|c|c|c|c|c|c|c|}
\hline \multirow{3}{*}{$\begin{array}{c}T=64 \\
\text { AUTOCO } \\
\text { LEVELS }(\delta)\end{array}$} & & \multicolumn{8}{|c|}{ COLLINEARITY $(\rho)$} \\
\hline & \multirow[t]{2}{*}{ Models } & \multicolumn{2}{|c|}{0.85} & \multicolumn{2}{|c|}{0.9} & \multicolumn{2}{|c|}{0.95} & \multicolumn{2}{|c|}{0.99} \\
\hline & & RMSE & MAE & RMSE & MAE & RMSE & MAE & RMSE & MAE \\
\hline \multirow{5}{*}{-0.99} & $\operatorname{VAR}(2)$ & 10.41419 & 7.75547 & 10.087394 & 7.099848 & 10.491135 & 8.167993 & 11.88104 & 10.17857 \\
\hline & BVAR1 & 7.879778 & 6.447886 & 7.559675 & 5.890353 & 7.554888 & 6.048543 & 8.649083 & 7.578297 \\
\hline & BVAR2 & 7.866083 & 6.442775 & 7.433292 & 5.760846 & 7.528471 & 6.008551 & 8.578764 & 7.519786 \\
\hline & BVAR3 & 7.669444 & 6.277168 & 7.286438 & 5.767222 & 7.346953 & 5.870459 & 8.202491 & 7.093822 \\
\hline & BVAR4 & 7.227625 & 5.847979 & 6.965772 & 5.417850 & 7.136368 & 5.663818 & 7.996016 & 6.881898 \\
\hline \multirow{5}{*}{-0.95} & $\operatorname{VAR}(2)$ & 5.515226 & 4.120926 & 5.486319 & 3.962742 & 5.676161 & 4.412665 & 6.800705 & 5.622775 \\
\hline & BVAR1 & 4.601067 & 3.744133 & 4.406236 & 3.518880 & 4.421690 & 3.527713 & 4.840530 & 4.044271 \\
\hline & BVAR2 & 4.534269 & 3.680055 & 4.345974 & 3.456424 & 4.354782 & 3.474134 & 4.791665 & 4.017778 \\
\hline & BVAR3 & 4.801356 & 4.000953 & 4.594733 & 3.780083 & 4.522771 & 3.662970 & 4.890993 & 4.091825 \\
\hline & BVAR4 & 4.591103 & 3.756402 & 4.433209 & 3.571501 & 4.439083 & 3.568040 & 4.873218 & 4.083426 \\
\hline \multirow{5}{*}{-0.9} & VAR(2) & 3.826275 & 2.825683 & 3.808973 & 2.734953 & 3.694262 & 2.865819 & 4.277465 & 3.498500 \\
\hline & BVAR1 & 3.534278 & 2.906586 & 3.362479 & 2.709000 & 3.335914 & 2.639133 & 3.602621 & 2.929623 \\
\hline & BVAR2 & 3.465804 & 2.838998 & 3.308653 & 2.659251 & 3.264809 & 2.583192 & 3.528374 & 2.874659 \\
\hline & BVAR3 & 3.808458 & 3.229122 & 3.605108 & 3.020947 & 3.471339 & 2.819498 & 3.608456 & 2.940455 \\
\hline & BVAR4 & 3.579565 & 2.983339 & 3.411765 & 2.807640 & 3.372967 & 2.712851 & 3.556532 & 2.899122 \\
\hline \multirow{5}{*}{-0.85} & VAR(2) & 2.786939 & 2.092726 & 2.927670 & 2.123987 & 2.956185 & 2.255688 & 3.292199 & 2.634300 \\
\hline & BVAR1 & 3.047345 & 2.501938 & 2.899607 & 2.334643 & 2.865764 & 2.254033 & 3.033291 & 2.420453 \\
\hline & BVAR2 & 2.980301 & 2.440607 & 2.818605 & 2.261592 & 2.773553 & 2.177460 & 2.940077 & 2.346569 \\
\hline & BVAR3 & 3.362504 & 2.860345 & 3.170085 & 2.667629 & 3.001249 & 2.431842 & 3.047829 & 2.436188 \\
\hline & BVAR4 & 3.131269 & 2.626230 & 2.977860 & 2.465824 & 2.878135 & 2.308610 & 2.974949 & 2.380450 \\
\hline \multirow{5}{*}{-0.8} & VAR(2) & 2.563719 & 1.866971 & 2.413336 & 1.770143 & 2.460161 & 1.865071 & 2.765376 & 2.174472 \\
\hline & BVAR1 & 2.765201 & 2.260260 & 2.637194 & 2.115751 & 2.596897 & 2.031406 & 2.705866 & 2.129469 \\
\hline & BVAR2 & 2.701178 & 2.205068 & 2.557811 & 2.044494 & 2.500364 & 1.948117 & 2.619745 & 2.055440 \\
\hline & BVAR3 & 3.123461 & 2.647115 & 2.927014 & 2.453578 & 2.737009 & 2.209300 & 2.722047 & 2.143268 \\
\hline & BVAR4 & 2.882840 & 2.416694 & 2.728992 & 2.256397 & 2.609029 & 2.081249 & 2.642185 & 2.077236 \\
\hline \multirow{5}{*}{0.8} & VAR(2) & 2.384340 & 1.847579 & 2.456279 & 1.916364 & 2.559812 & 2.006761 & 2.676730 & 2.104725 \\
\hline & BVAR1 & 2.926542 & 2.373617 & 2.971888 & 2.414482 & 3.021903 & 2.452398 & 3.027127 & 2.440837 \\
\hline & BVAR2 & 2.850652 & 2.299951 & 2.891917 & 2.335971 & 2.958457 & 2.388264 & 2.972621 & 2.387389 \\
\hline & BVAR3 & 2.82495 & 2.27897 & 2.836890 & 2.277432 & 2.897326 & 2.317965 & 2.914402 & 2.315717 \\
\hline & BVAR4 & 2.69156 & 2.14932 & 2.710707 & 2.157766 & 2.764935 & 2.193781 & 2.809153 & 2.217734 \\
\hline \multirow{5}{*}{0.85} & VAR(2) & 2.788461 & 2.171616 & 2.851412 & 2.260241 & 2.911770 & 2.326237 & 3.07246 & 2.46561 \\
\hline & BVAR1 & 3.195656 & 2.605438 & 3.243095 & 2.649101 & 3.295623 & 2.693263 & 3.343212 & 2.722561 \\
\hline & BVAR2 & 3.126292 & 2.538026 & 3.193553 & 2.599884 & 3.240708 & 2.638987 & 3.297007 & 2.674201 \\
\hline & BVAR3 & 3.050595 & 2.468853 & 3.088025 & 2.493688 & 3.163816 & 2.549461 & 3.192363 & 2.564826 \\
\hline & BVAR4 & 2.919833 & 2.347208 & 2.969034 & 2.381272 & 3.019581 & 2.417416 & 3.086137 & 2.467136 \\
\hline \multirow{5}{*}{0.9} & VAR(2) & 3.623288 & 2.868317 & 3.527514 & 2.855164 & 4.027746 & 3.218235 & 4.061071 & 3.324801 \\
\hline & BVAR1 & 3.649271 & 2.990935 & 3.721894 & 3.065972 & 3.787166 & 3.122032 & 3.897328 & 3.214863 \\
\hline & BVAR2 & 3.588610 & 2.934248 & 3.651061 & 2.997455 & 3.720466 & 3.062137 & 3.834919 & 3.157984 \\
\hline & BVAR3 & 3.448331 & 2.809900 & 3.519245 & 2.868675 & 3.589405 & 2.927547 & 3.636149 & 2.962102 \\
\hline & BVAR4 & 3.326695 & 2.698520 & 3.397685 & 2.755271 & 3.462768 & 2.811275 & 3.565531 & 2.896450 \\
\hline \multirow{5}{*}{0.95} & VAR(2) & 5.815011 & 4.565871 & 6.010590 & 4.823818 & 6.359985 & 5.238172 & 6.385236 & 5.332214 \\
\hline & BVAR1 & 4.473675 & 3.688176 & 4.589992 & 3.811018 & 4.720950 & 3.944863 & 4.961381 & 4.157459 \\
\hline & BVAR2 & 4.453470 & 3.668865 & 4.534502 & 3.760519 & 4.706124 & 3.929342 & 4.849424 & 4.058908 \\
\hline & BVAR3 & 4.244755 & 3.495875 & 4.318832 & 3.569387 & 4.431429 & 3.678013 & 4.601010 & 3.833053 \\
\hline & BVAR4 & 4.125876 & 3.388459 & 4.240229 & 3.495942 & 4.372050 & 3.623511 & 4.508561 & 3.744225 \\
\hline \multirow{5}{*}{0.99} & VAR(2) & 10.356067 & 8.039591 & 10.404217 & 8.460912 & 12.255648 & 9.648472 & 11.684346 & 9.806151 \\
\hline & BVAR1 & 5.982988 & 4.959825 & 6.135503 & 5.141708 & 6.333499 & 5.366900 & 6.664584 & 5.676046 \\
\hline & BVAR2 & 6.004868 & 4.995543 & 6.119139 & 5.145547 & 6.305826 & 5.346008 & 6.678079 & 5.711101 \\
\hline & BVAR3 & 5.770993 & 4.806477 & 5.864026 & 4.924136 & 6.158097 & 5.219477 & 6.343388 & 5.409572 \\
\hline & BVAR4 & 5.746963 & 4.791406 & 5.811905 & 4.891243 & 6.034546 & 5.108078 & 6.298889 & 5.371982 \\
\hline
\end{tabular}


Table A5. The RMSE and MAE values of the model for $T=128$.

\begin{tabular}{|c|c|c|c|c|c|c|c|c|c|c|c|c|c|}
\hline \multicolumn{2}{|l|}{$T=128$} & \multicolumn{12}{|c|}{ COLLINERITY $(\rho)$} \\
\hline \multirow{2}{*}{$\begin{array}{l}\text { AUTOCO } \\
\text { LEVELS } \\
(\delta)\end{array}$} & \multirow[t]{2}{*}{ Models } & \multicolumn{2}{|c|}{-0.99} & \multicolumn{2}{|c|}{-0.95} & \multicolumn{2}{|c|}{-0.9} & \multicolumn{2}{|c|}{-0.85} & \multicolumn{2}{|c|}{-0.8} & \multicolumn{2}{|c|}{0.8} \\
\hline & & RMSE & MAE & RMSE & MAE & RMSE & MAE & RMSE & MAE & RMSE & MAE & RMSE & MAE \\
\hline
\end{tabular}

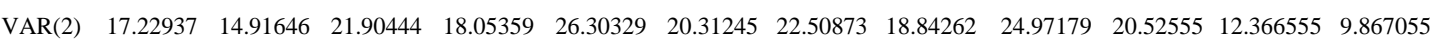
$\begin{array}{lllllllllllll}\text { BVAR1 } & 13.23589 & 11.46483 & 15.27269 & 12.99190 & 16.48863 & 13.79484 & 17.25900 & 14.30382 & 17.90545 & 14.70387 & 9.753913 & 8.111029\end{array}$ $\begin{array}{lllllllllllll}\text { BVAR2 } & 13.17653 & 11.40185 & 15.11556 & 12.82739 & 16.1352 & 13.4810 & 16.85251 & 13.90399 & 17.42538 & 14.27669 & 9.570891 & 7.934374\end{array}$

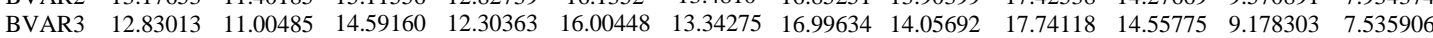

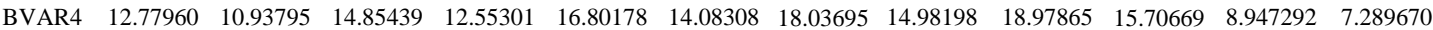

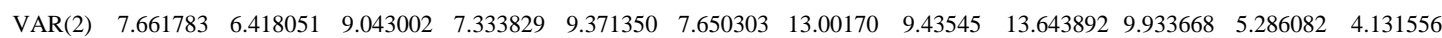
$\begin{array}{lllllllllllll}\text { BVAR1 } & 6.370249 & 5.269217 & 7.332931 & 5.968955 & 8.031373 & 6.448491 & 8.500655 & 6.762176 & 8.895059 & 7.019988 & 5.142322 & 4.229382\end{array}$ $\begin{array}{llllllllllllll}\text { BVAR2 } & 6.372227 & 5.266374 & 7.333282 & 5.966015 & 8.052061 & 6.481550 & 8.517979 & 6.787717 & 8.921330 & 7.061148 & 4.918230 & 3.985872\end{array}$ $\begin{array}{llllllllllllll}\text { BVAR3 } & 6.500161 & 5.386179 & 7.476272 & 6.102912 & 8.107454 & 6.535236 & 8.615760 & 6.889719 & 8.936636 & 7.099140 & 4.985552 & 4.054149\end{array}$

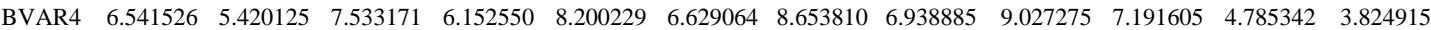

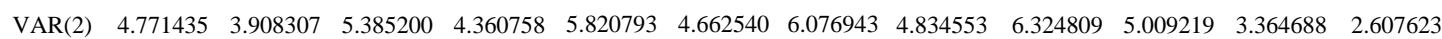
$\begin{array}{lllllllllllll}\text { BVAR1 } & 4.362781 & 3.551253 & 4.980801 & 3.993930 & 5.469379 & 4.323643 & 5.827049 & 4.557198 & 6.126422 & 4.753069 & 3.947152 & 3.308077\end{array}$ $\begin{array}{lllllllllllll}\text { BVAR2 } & 4.337249 & 3.526718 & 4.991824 & 4.002132 & 5.429540 & 4.292408 & 5.807013 & 4.553049 & 6.074564 & 4.723629 & 3.660195 & 3.018613\end{array}$ $\begin{array}{lllllllllllll}\text { BVAR3 } & 4.393323 & 3.577711 & 5.030188 & 4.038974 & 5.458506 & 4.332745 & 5.764595 & 4.531048 & 6.029237 & 4.711636 & 3.745564 & 3.103055\end{array}$

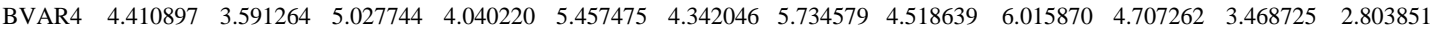

$\begin{array}{lllllllllllll}\text { VAR(2) } & 3.680692 & 2.967355 & 4.095767 & 3.276760 & 4.422976 & 3.506239 & 4.615566 & 3.638250 & 4.777651 & 3.748394 & 2.669983 & 2.050323\end{array}$ $\begin{array}{lllllllllllll}\text { BVAR1 } & 3.454091 & 2.779658 & 3.938882 & 3.133889 & 4.342657 & 3.406856 & 4.665832 & 3.623807 & 4.899277 & 3.774418 & 3.468023 & 2.926042\end{array}$ $\begin{array}{llllllllllllll}\text { BVAR2 } & 3.461064 & 2.789749 & 3.944574 & 3.137978 & 4.320403 & 3.395097 & 4.595528 & 3.575939 & 4.810876 & 3.715687 & 3.162908 & 2.633049\end{array}$ $\begin{array}{lllllllllllll}\text { BVAR3 } & 3.479713 & 2.803527 & 3.939841 & 3.141700 & 4.285884 & 3.380606 & 4.562836 & 3.561649 & 4.765831 & 3.697382 & 3.244371 & 2.718677\end{array}$

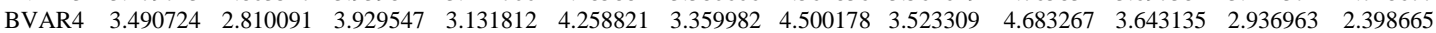

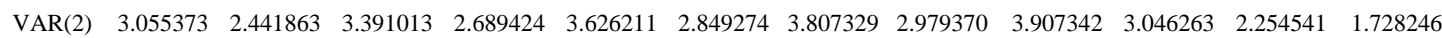
$\begin{array}{lllllllllllll}\text { BVAR1 } & 2.944984 & 2.352791 & 3.331575 & 2.633942 & 3.698470 & 2.890197 & 3.983771 & 3.079893 & 4.212778 & 3.231453 & 3.191163 & 2.692211\end{array}$ $\begin{array}{lllllllllllll}\text { BVAR2 } & 2.944741 & 2.353353 & 3.348329 & 2.646829 & 3.675676 & 2.875018 & 3.910292 & 3.027415 & 4.116140 & 3.165877 & 2.874961 & 2.401644\end{array}$ $\begin{array}{lllllllllllll}\text { BVAR3 } & 2.955164 & 2.360062 & 3.323106 & 2.633752 & 3.636439 & 2.852393 & 3.849498 & 2.993170 & 4.046711 & 3.126502 & 2.979943 & 2.511278\end{array}$ $\begin{array}{lllllllllllll}\text { BVAR4 } & 2.953468 & 2.359096 & 3.311263 & 2.622841 & 3.579884 & 2.809669 & 3.777316 & 2.943939 & 3.924045 & 3.038138 & 2.645861 & 2.174288\end{array}$

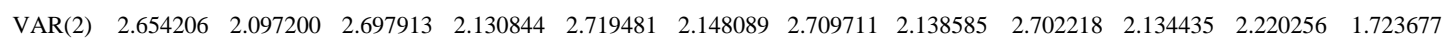

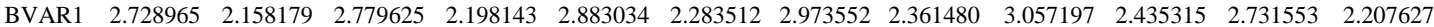

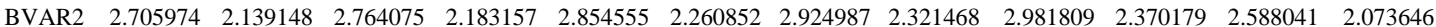
$\begin{array}{lllllllllllll}\text { BVAR3 } & 2.665213 & 2.105128 & 2.68787 & 2.12321 & 2.731597 & 2.158491 & 2.781588 & 2.200491 & 2.826168 & 2.237295 & 2.673238 & 2.171811\end{array}$

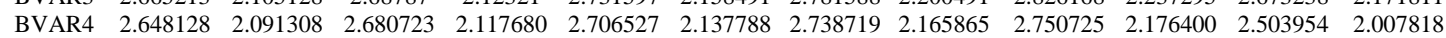

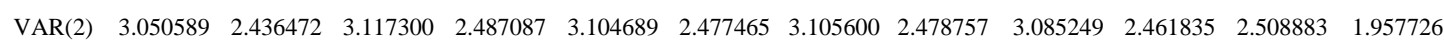
$\begin{array}{lllllllllllll}\text { BVAR1 } & 3.100007 & 2.473666 & 3.139194 & 2.500250 & 3.218102 & 2.566508 & 3.307878 & 2.642758 & 3.371854 & 2.697790 & 2.996137 & 2.420239\end{array}$ $\begin{array}{lllllllllllll}\text { BVAR2 } & 3.071986 & 2.449269 & 3.117831 & 2.485876 & 3.184090 & 2.539957 & 3.247531 & 2.591621 & 3.290611 & 2.630079 & 2.858626 & 2.291587\end{array}$ $\begin{array}{lllllllllllll}\text { BVAR3 } & 3.004581 & 2.393383 & 3.043114 & 2.423359 & 3.073587 & 2.449375 & 3.119071 & 2.484352 & 3.138801 & 2.502352 & 2.911674 & 2.362096\end{array}$ $\begin{array}{lllllllllllll}\text { BVAR4 } & 2.985086 & 2.377309 & 3.017451 & 2.402853 & 3.057904 & 2.435876 & 3.066501 & 2.441635 & 3.087073 & 2.460630 & 2.748926 & 2.206288\end{array}$

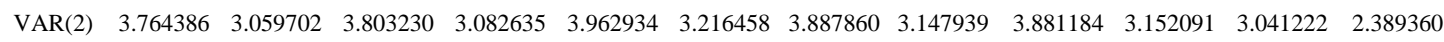
$\begin{array}{lllllllllllll}\text { BVAR1 } & 3.740808 & 3.022111 & 3.765262 & 3.036083 & 3.829513 & 3.084710 & 3.897016 & 3.144560 & 3.933268 & 3.171288 & 3.457976 & 2.795069\end{array}$ $\begin{array}{lllllllllllll}\text { BVAR2 } & 3.680259 & 2.966716 & 3.729521 & 3.006810 & 3.794741 & 3.057041 & 3.827421 & 3.085142 & 3.866029 & 3.113105 & 3.326255 & 2.673346\end{array}$ $\begin{array}{lllllllllllll}\text { BVAR3 } & 3.594659 & 2.898046 & 3.626014 & 2.919360 & 3.666482 & 2.951201 & 3.692122 & 2.972574 & 3.709252 & 2.986345 & 3.315510 & 2.687656\end{array}$

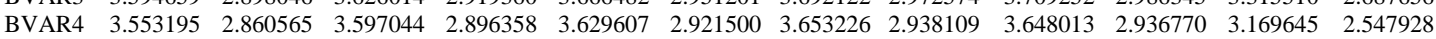

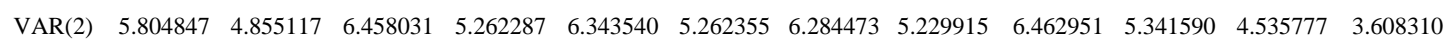
$\begin{array}{llllllllllllll}\text { BVAR1 } & 5.157735 & 4.250161 & 5.175174 & 4.255861 & 5.301088 & 4.359783 & 5.270171 & 4.334131 & 5.269785 & 4.325537 & 4.506465 & 3.660309\end{array}$ $\begin{array}{lllllllllllll}\text { BVAR2 } & 5.074068 & 4.175784 & 5.13902 & 4.22378 & 5.167625 & 4.244808 & 5.189649 & 4.258897 & 5.197418 & 4.263038 & 4.393451 & 3.555035\end{array}$ $\begin{array}{llllllllllllll}\text { BVAR3 } & 4.884419 & 4.013207 & 4.951390 & 4.063292 & 5.035572 & 4.132024 & 5.037693 & 4.129435 & 5.062795 & 4.150245 & 4.289787 & 3.489605\end{array}$

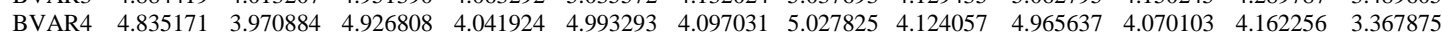

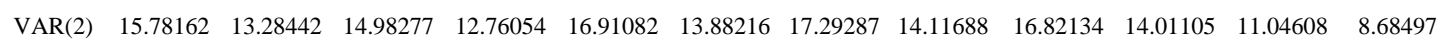

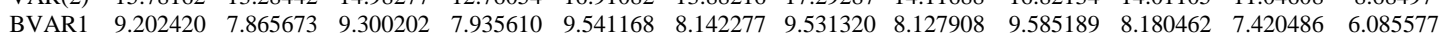

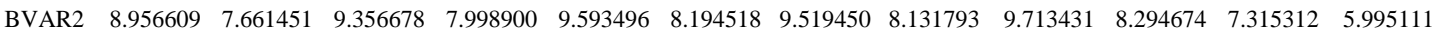
$\begin{array}{lllllllllllll}\text { BVAR3 } & 8.837134 & 7.555928 & 9.218690 & 7.875251 & 9.303724 & 7.946998 & 9.380312 & 8.011799 & 9.448674 & 8.069179 & 7.143228 & 5.861300\end{array}$

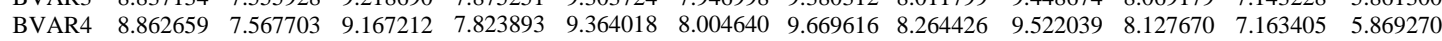




\section{Continued}

\begin{tabular}{|c|c|c|c|c|c|c|c|c|c|}
\hline \multirow{3}{*}{$\begin{array}{c}\text { AUTOCO } \\
\text { LEVELS }(\delta)\end{array}$} & \multirow{3}{*}{ Models } & \multicolumn{8}{|c|}{ COLLINEARITY $(\rho)$} \\
\hline & & \multicolumn{2}{|c|}{0.85} & \multicolumn{2}{|c|}{0.9} & \multicolumn{2}{|c|}{0.95} & \multicolumn{2}{|c|}{0.99} \\
\hline & & RMSE & MAE & RMSE & MAE & RMSE & MAE & RMSE & MAE \\
\hline-0.99 & $\begin{array}{l}\text { VAR(2) } \\
\text { BVAR1 } \\
\text { BVAR2 } \\
\text { BVAR3 } \\
\text { BVAR4 }\end{array}$ & $\begin{array}{c}11.945267 \\
9.179577 \\
9.037003 \\
8.707451 \\
8.462042\end{array}$ & $\begin{array}{l}8.861576 \\
7.320466 \\
7.133194 \\
6.855307 \\
6.577923\end{array}$ & $\begin{array}{c}12.795160 \\
8.819682 \\
8.697249 \\
8.478398 \\
8.258024\end{array}$ & $\begin{array}{l}8.681859 \\
6.748474 \\
6.544172 \\
6.397376 \\
6.126412\end{array}$ & $\begin{array}{l}11.85843 \\
8.942636 \\
8.979889 \\
8.752523 \\
8.563999\end{array}$ & $\begin{array}{c}9.25846 \\
7.083999 \\
7.091445 \\
6.889068 \\
6.706095\end{array}$ & $\begin{array}{c}13.42278 \\
10.185347 \\
10.180364 \\
9.9200541 \\
9.799041\end{array}$ & $\begin{array}{l}11.42555 \\
8.754547 \\
8.740516 \\
8.442729 \\
8.330114\end{array}$ \\
\hline-0.95 & $\begin{array}{l}\text { VAR(2) } \\
\text { BVAR1 } \\
\text { BVAR2 } \\
\text { BVAR3 } \\
\text { BVAR4 }\end{array}$ & $\begin{array}{l}5.015870 \\
4.836986 \\
4.653996 \\
4.726680 \\
4.575834\end{array}$ & $\begin{array}{l}3.725254 \\
3.953661 \\
3.708048 \\
3.790807 \\
3.568500\end{array}$ & $\begin{array}{l}5.203213 \\
4.644456 \\
4.471611 \\
4.585791 \\
4.471021\end{array}$ & $\begin{array}{l}3.696729 \\
3.728887 \\
3.492956 \\
3.614554 \\
3.415283\end{array}$ & $\begin{array}{l}5.426878 \\
4.642191 \\
4.550617 \\
4.633726 \\
4.567308\end{array}$ & $\begin{array}{l}4.146475 \\
3.679435 \\
3.572558 \\
3.660861 \\
3.573860\end{array}$ & $\begin{array}{l}6.062297 \\
5.055933 \\
5.019349 \\
5.112308 \\
5.125466\end{array}$ & $\begin{array}{l}4.980789 \\
4.151075 \\
4.122661 \\
4.202335 \\
4.213588\end{array}$ \\
\hline-0.9 & $\begin{array}{l}\text { VAR(2) } \\
\text { BVAR1 } \\
\text { BVAR2 } \\
\text { BVAR3 } \\
\text { BVAR4 }\end{array}$ & $\begin{array}{l}3.264372 \\
3.714970 \\
3.458167 \\
3.551787 \\
3.306702\end{array}$ & $\begin{array}{l}2.433065 \\
3.118219 \\
2.836522 \\
2.938455 \\
2.644407\end{array}$ & $\begin{array}{l}3.348979 \\
3.504954 \\
3.298093 \\
3.400993 \\
3.221331\end{array}$ & $\begin{array}{l}2.426611 \\
2.898950 \\
2.657997 \\
2.777254 \\
2.533225\end{array}$ & $\begin{array}{l}3.336172 \\
3.379134 \\
3.262088 \\
3.369001 \\
3.249320\end{array}$ & $\begin{array}{l}2.564084 \\
2.701847 \\
2.578714 \\
2.689641 \\
2.549904\end{array}$ & $\begin{array}{l}3.771946 \\
3.563928 \\
3.496387 \\
3.597387 \\
3.544725\end{array}$ & $\begin{array}{l}3.064766 \\
2.875681 \\
2.821228 \\
2.904003 \\
2.859338\end{array}$ \\
\hline-0.85 & $\begin{array}{l}\text { VAR(2) } \\
\text { BVAR1 } \\
\text { BVAR2 } \\
\text { BVAR3 } \\
\text { BVAR4 }\end{array}$ & $\begin{array}{l}2.567553 \\
3.244740 \\
2.972959 \\
3.075161 \\
2.801145\end{array}$ & $\begin{array}{l}1.921773 \\
2.745461 \\
2.470611 \\
2.582631 \\
2.275310\end{array}$ & $\begin{array}{l}2.541639 \\
3.016541 \\
2.809365 \\
2.920878 \\
2.706614\end{array}$ & $\begin{array}{l}1.883283 \\
2.519631 \\
2.294248 \\
2.421964 \\
2.165321\end{array}$ & $\begin{array}{l}2.626303 \\
2.848164 \\
2.728691 \\
2.838472 \\
2.700311\end{array}$ & $\begin{array}{l}2.007082 \\
2.288249 \\
2.166030 \\
2.285118 \\
2.127450\end{array}$ & $\begin{array}{l}3.018725 \\
2.915867 \\
2.853590 \\
2.933008 \\
2.886501\end{array}$ & $\begin{array}{l}2.408264 \\
2.326463 \\
2.277067 \\
2.345572 \\
2.303731\end{array}$ \\
\hline-0.8 & $\begin{array}{l}\text { VAR(2) } \\
\text { BVAR1 } \\
\text { BVAR2 } \\
\text { BVAR3 } \\
\text { BVAR4 }\end{array}$ & $\begin{array}{l}2.196236 \\
2.973229 \\
2.696030 \\
2.819576 \\
2.523249\end{array}$ & $\begin{array}{l}1.646146 \\
2.513734 \\
2.249508 \\
2.381977 \\
2.066675\end{array}$ & $\begin{array}{l}2.183864 \\
2.747384 \\
2.541589 \\
2.659572 \\
2.427958\end{array}$ & $\begin{array}{l}1.622701 \\
2.294729 \\
2.084607 \\
2.219254 \\
1.958831\end{array}$ & $\begin{array}{l}2.265826 \\
2.547671 \\
2.420984 \\
2.549656 \\
2.394875\end{array}$ & $\begin{array}{l}1.723982 \\
2.048411 \\
1.922024 \\
2.059531 \\
1.891775\end{array}$ & $\begin{array}{l}2.503623 \\
2.548932 \\
2.472584 \\
2.567721 \\
2.501872\end{array}$ & $\begin{array}{l}1.975381 \\
2.015365 \\
1.952281 \\
2.034474 \\
1.978518\end{array}$ \\
\hline 0.8 & $\begin{array}{l}\text { VAR(2) } \\
\text { BVAR1 } \\
\text { BVAR2 } \\
\text { BVAR3 } \\
\text { BVAR4 }\end{array}$ & $\begin{array}{l}2.269407 \\
2.752129 \\
2.597009 \\
2.658591 \\
2.504221\end{array}$ & $\begin{array}{l}1.772237 \\
2.217063 \\
2.073938 \\
2.142858 \\
1.997949\end{array}$ & $\begin{array}{l}2.338102 \\
2.764549 \\
2.631648 \\
2.638225 \\
2.514020\end{array}$ & $\begin{array}{l}1.835759 \\
2.221370 \\
2.096248 \\
2.111596 \\
1.997147\end{array}$ & $\begin{array}{l}2.422130 \\
2.787988 \\
2.669347 \\
2.644182 \\
2.546331\end{array}$ & $\begin{array}{l}1.909445 \\
2.232614 \\
2.122810 \\
2.103946 \\
2.017356\end{array}$ & $\begin{array}{l}2.532666 \\
2.803706 \\
2.700260 \\
2.688484 \\
2.600767\end{array}$ & $\begin{array}{l}2.001992 \\
2.237125 \\
2.143836 \\
2.131183 \\
2.058269\end{array}$ \\
\hline 0.85 & $\begin{array}{l}\text { VAR(2) } \\
\text { BVAR1 } \\
\text { BVAR2 } \\
\text { BVAR3 } \\
\text { BVAR4 }\end{array}$ & $\begin{array}{c}2.567649 \\
3.021006 \\
2.880938 \\
2.89842 \\
2.760013\end{array}$ & $\begin{array}{c}2.018788 \\
2.436361 \\
2.306900 \\
2.33901 \\
2.208032\end{array}$ & $\begin{array}{l}2.644502 \\
3.053786 \\
2.925930 \\
2.905182 \\
2.787058\end{array}$ & $\begin{array}{l}2.093358 \\
2.459015 \\
2.342555 \\
2.331787 \\
2.224654\end{array}$ & $\begin{array}{l}2.742656 \\
3.094658 \\
2.979290 \\
2.928327 \\
2.830560\end{array}$ & $\begin{array}{l}2.183696 \\
2.490691 \\
2.385108 \\
2.340648 \\
2.258261\end{array}$ & $\begin{array}{l}2.870326 \\
3.127300 \\
3.042191 \\
2.992054 \\
2.914858\end{array}$ & $\begin{array}{l}2.291302 \\
2.512577 \\
2.435299 \\
2.389238 \\
2.326037\end{array}$ \\
\hline 0.9 & $\begin{array}{l}\text { VAR(2) } \\
\text { BVAR1 } \\
\text { BVAR2 } \\
\text { BVAR3 } \\
\text { BVAR4 }\end{array}$ & $\begin{array}{l}3.137792 \\
3.486373 \\
3.363206 \\
3.327585 \\
3.200512\end{array}$ & $\begin{array}{l}2.487511 \\
2.818011 \\
2.705538 \\
2.691013 \\
2.571322\end{array}$ & $\begin{array}{l}3.191229 \\
3.553037 \\
3.421814 \\
3.357432 \\
3.239301\end{array}$ & $\begin{array}{l}2.554643 \\
2.873810 \\
2.758570 \\
2.706657 \\
2.602163\end{array}$ & $\begin{array}{l}3.344238 \\
3.626711 \\
3.502733 \\
3.427382 \\
3.324481\end{array}$ & $\begin{array}{l}2.700335 \\
2.938518 \\
2.828356 \\
2.764448 \\
2.672839\end{array}$ & $\begin{array}{l}3.564692 \\
3.692615 \\
3.596625 \\
3.530147 \\
3.441597\end{array}$ & $\begin{array}{l}2.890408 \\
2.991344 \\
2.910104 \\
2.848907 \\
2.771189\end{array}$ \\
\hline 0.95 & $\begin{array}{l}\text { VAR(2) } \\
\text { BVAR1 } \\
\text { BVAR2 } \\
\text { BVAR3 } \\
\text { BVAR4 }\end{array}$ & $\begin{array}{l}4.920988 \\
4.595254 \\
4.424569 \\
4.343997 \\
4.223473\end{array}$ & $\begin{array}{l}3.944748 \\
3.750031 \\
3.596945 \\
3.540982 \\
3.429542\end{array}$ & $\begin{array}{l}4.990236 \\
4.668532 \\
4.565638 \\
4.421092 \\
4.331716\end{array}$ & $\begin{array}{l}4.055321 \\
3.825789 \\
3.732137 \\
3.612574 \\
3.532617\end{array}$ & $\begin{array}{l}5.399281 \\
4.792548 \\
4.682770 \\
4.482806 \\
4.414773\end{array}$ & $\begin{array}{l}4.430346 \\
3.941870 \\
3.845421 \\
3.669919 \\
3.608953\end{array}$ & $\begin{array}{l}5.950925 \\
4.948153 \\
4.869442 \\
4.728360 \\
4.636423\end{array}$ & $\begin{array}{l}4.832073 \\
4.085370 \\
4.013891 \\
3.885237 \\
3.809460\end{array}$ \\
\hline 0.99 & $\begin{array}{l}\text { VAR(2) } \\
\text { BVAR1 } \\
\text { BVAR2 } \\
\text { BVAR3 } \\
\text { BVAR4 }\end{array}$ & $\begin{array}{c}11.455568 \\
7.426306 \\
7.434341 \\
7.384678 \\
7.364817\end{array}$ & $\begin{array}{l}9.209777 \\
6.151560 \\
6.160498 \\
6.145512 \\
6.124219\end{array}$ & $\begin{array}{c}11.893301 \\
7.688341 \\
7.577398 \\
7.656384 \\
7.471577\end{array}$ & $\begin{array}{l}9.775085 \\
6.443587 \\
6.343787 \\
6.421955 \\
6.273514\end{array}$ & $\begin{array}{l}13.54296 \\
7.999046 \\
8.036029 \\
7.786935 \\
7.717748\end{array}$ & $\begin{array}{l}11.18886 \\
6.774294 \\
6.824181 \\
6.602134 \\
6.542996\end{array}$ & $\begin{array}{l}13.50771 \\
8.478266 \\
8.367662 \\
8.226474 \\
8.117583\end{array}$ & $\begin{array}{l}11.46993 \\
7.229107 \\
7.135695 \\
7.007422 \\
6.914989\end{array}$ \\
\hline
\end{tabular}


Table A6. The RMSE and MAE values of the model for $T=256$.

\begin{tabular}{|c|c|c|c|c|c|c|c|c|c|c|c|c|c|}
\hline \multicolumn{2}{|l|}{$T=256$} & \multicolumn{12}{|c|}{ COLLINERITY $(\rho)$} \\
\hline \multirow{2}{*}{$\begin{array}{l}\text { AUTOCO } \\
\text { LEVELS }(\delta)\end{array}$} & \multirow[t]{2}{*}{ Models } & \multicolumn{2}{|c|}{-0.99} & \multicolumn{2}{|c|}{-0.95} & \multicolumn{2}{|c|}{-0.9} & \multicolumn{2}{|c|}{-0.85} & \multicolumn{2}{|c|}{-0.8} & \multicolumn{2}{|c|}{0.8} \\
\hline & & RMSE & MAE & RMSE & MAE & RMSE & MAE & RMSE & MAE & RMSE & MAE & RMSE & MAE \\
\hline
\end{tabular}

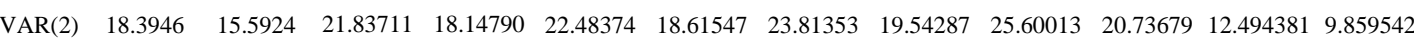

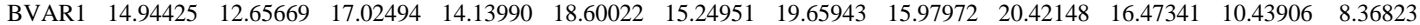

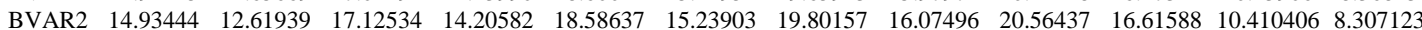
$\begin{array}{lllllllllllll}\text { BVAR3 } & 15.29943 & 12.92135 & 17.63730 & 14.67914 & 19.77131 & 16.28300 & 20.94188 & 17.13175 & 22.33767 & 18.19815 & 10.345147 & 8.217326\end{array}$

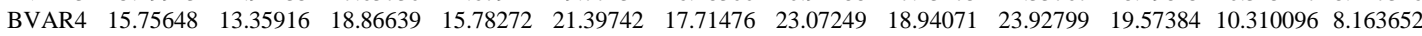

$\begin{array}{lllllllllllll}\text { VAR(2) } & 7.218011 & 5.847984 & 8.030496 & 6.467167 & 8.668218 & 6.894023 & 9.425220 & 7.393212 & 9.495944 & 7.448484 & 4.879222 & 3.746798\end{array}$ $\begin{array}{lllllllllllll}\text { BVAR1 } & 6.568200 & 5.334939 & 7.544869 & 6.031629 & 8.240385 & 6.509597 & 8.704961 & 6.810649 & 9.101330 & 7.074803 & 5.004025 & 4.017078\end{array}$

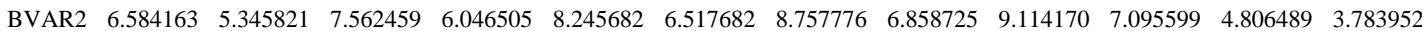
$\begin{array}{llllllllllllll}\text { BVAR3 } & 6.682222 & 5.425272 & 7.657178 & 6.127892 & 8.348658 & 6.612652 & 8.874156 & 6.963380 & 9.160590 & 7.142968 & 4.848034 & 3.810604\end{array}$

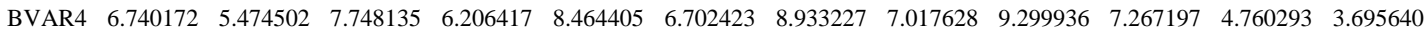

$\begin{array}{lllllllllllll}\text { VAR(2) } & 4.548186 & 3.661812 & 5.163005 & 4.096899 & 5.594353 & 4.395694 & 5.886288 & 4.588031 & 6.081617 & 4.714155 & 3.237686 & 2.479712\end{array}$ $\begin{array}{lllllllllllll}\text { BVAR1 } & 4.410247 & 3.547042 & 5.058111 & 4.006136 & 5.510708 & 4.313132 & 5.879078 & 4.561168 & 6.107963 & 4.706490 & 3.715527 & 3.061258\end{array}$ $\begin{array}{lllllllllllll}\text { BVAR2 } & 4.435212 & 3.567766 & 5.061483 & 4.014110 & 5.506703 & 4.315287 & 5.805862 & 4.508307 & 6.060609 & 4.674692 & 3.435002 & 2.751851\end{array}$ $\begin{array}{lllllllllllll}\text { BVAR3 } & 4.458940 & 3.587023 & 5.064372 & 4.019333 & 5.502904 & 4.316899 & 5.807830 & 4.515899 & 6.046962 & 4.674377 & 3.432424 & 2.744897\end{array}$

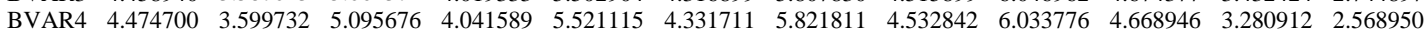

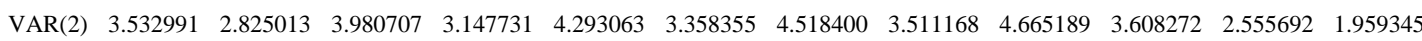
$\begin{array}{lllllllllllll}\text { BVAR1 } & 3.472187 & 2.780203 & 3.960429 & 3.127099 & 4.33727 & 3.38154 & 4.606734 & 3.559702 & 4.835993 & 3.709757 & 3.192449 & 2.670262\end{array}$ $\begin{array}{lllllllllllll}\text { BVAR2 } & 3.471253 & 2.777734 & 3.949921 & 3.118628 & 4.298323 & 3.355954 & 4.534228 & 3.508752 & 4.746031 & 3.650103 & 2.867236 & 2.330504\end{array}$ $\begin{array}{lllllllllllll}\text { BVAR3 } & 3.479885 & 2.783814 & 3.952865 & 3.125543 & 4.273287 & 3.341796 & 4.518117 & 3.506743 & 4.692695 & 3.619978 & 2.866857 & 2.324536\end{array}$ $\begin{array}{lllllllllllll}\text { BVAR4 } & 3.479531 & 2.783306 & 3.938482 & 3.113071 & 4.267845 & 3.337840 & 4.485010 & 3.482246 & 4.659591 & 3.600075 & 2.675640 & 2.113137\end{array}$

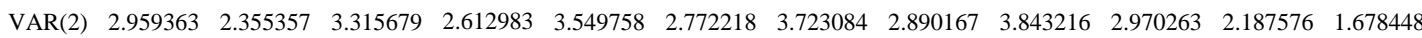

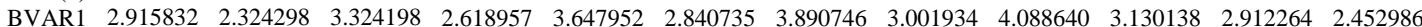
$\begin{array}{lllllllllllll}\text { BVAR2 } & 2.927606 & 2.333337 & 3.315964 & 2.612976 & 3.588153 & 2.796431 & 3.805687 & 2.940283 & 3.972382 & 3.050013 & 2.559063 & 2.097676\end{array}$ $\begin{array}{llllllllllllll}\text { BVAR3 } & 2.934119 & 2.337806 & 3.294327 & 2.598272 & 3.573094 & 2.791090 & 3.760643 & 2.914655 & 3.905587 & 3.009587 & 2.553443 & 2.088967\end{array}$ $\begin{array}{lllllllllllll}\text { BVAR4 } & 2.927636 & 2.332091 & 3.283496 & 2.589628 & 3.538577 & 2.764798 & 3.717292 & 2.883763 & 3.861316 & 2.980157 & 2.338844 & 1.859071\end{array}$

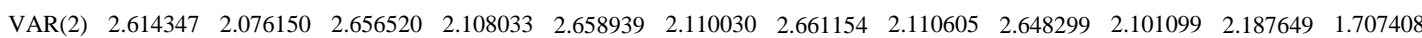

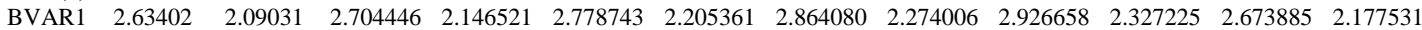

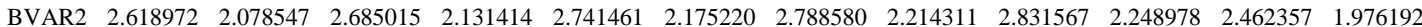
$\begin{array}{lllllllllllll}\text { BVAR3 } & 2.613630 & 2.076179 & 2.660450 & 2.112121 & 2.701794 & 2.144857 & 2.727534 & 2.165545 & 2.748650 & 2.183494 & 2.507330 & 2.024276\end{array}$ $\begin{array}{lllllllllllll}\text { BVAR4 } & 2.602326 & 2.066396 & 2.647095 & 2.101200 & 2.669346 & 2.118583 & 2.677478 & 2.125707 & 2.682583 & 2.129126 & 2.338754 & 1.858694\end{array}$

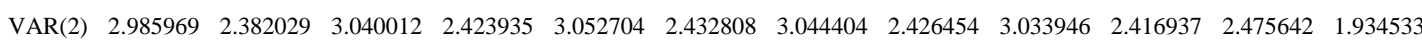

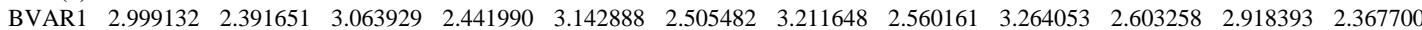

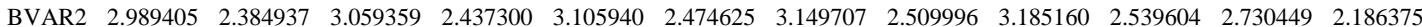
$\begin{array}{llllllllllllll}\text { BVAR3 } & 2.968755 & 2.368240 & 3.027330 & 2.413446 & 3.069040 & 2.447164 & 3.087766 & 2.462067 & 3.106623 & 2.476152 & 2.761086 & 2.223583\end{array}$ $\begin{array}{lllllllllllll}\text { BVAR4 } & 2.967988 & 2.367233 & 3.018417 & 2.407020 & 3.043088 & 2.426606 & 3.047539 & 2.428791 & 3.046364 & 2.428108 & 2.609852 & 2.072087\end{array}$

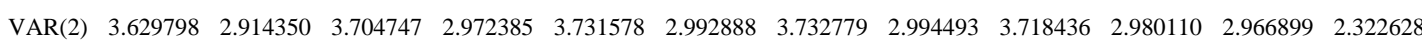
$\begin{array}{llllllllllllll}\text { BVAR1 } & 3.628762 & 2.911740 & 3.699430 & 2.965376 & 3.777637 & 3.026668 & 3.816494 & 3.055784 & 3.854139 & 3.086413 & 3.361970 & 2.712292\end{array}$ $\begin{array}{lllllllllllll}\text { BVAR2 } & 3.615355 & 2.900748 & 3.683542 & 2.952034 & 3.730334 & 2.987159 & 3.769586 & 3.018176 & 3.786167 & 3.033074 & 3.191064 & 2.548203\end{array}$ $\begin{array}{lllllllllllll}\text { BVAR3 } & 3.587281 & 2.877089 & 3.654505 & 2.931531 & 3.695062 & 2.961888 & 3.703219 & 2.968410 & 3.726128 & 2.985445 & 3.208336 & 2.574259\end{array}$

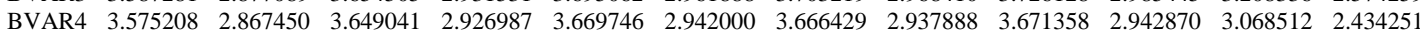

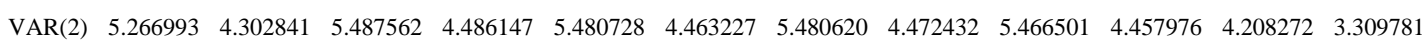
$\begin{array}{llllllllllllll}\text { BVAR1 } & 5.024042 & 4.073294 & 5.120990 & 4.146785 & 5.206815 & 4.217585 & 5.226842 & 4.226614 & 5.239742 & 4.238858 & 4.396909 & 3.533885\end{array}$ $\begin{array}{lllllllllllll}\text { BVAR2 } & 4.997996 & 4.053201 & 5.058593 & 4.096847 & 5.140416 & 4.158530 & 5.165222 & 4.177438 & 5.170006 & 4.182593 & 4.260906 & 3.401164\end{array}$ $\begin{array}{lllllllllllll}\text { BVAR3 } & 4.938789 & 4.002242 & 5.039830 & 4.080811 & 5.093600 & 4.125176 & 5.106001 & 4.131230 & 5.111003 & 4.134270 & 4.232342 & 3.382767\end{array}$

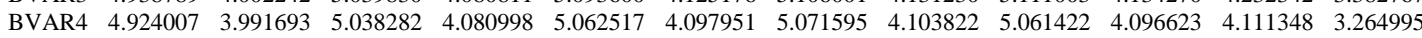

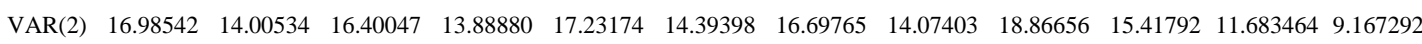

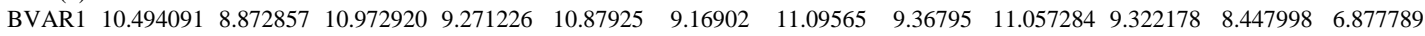

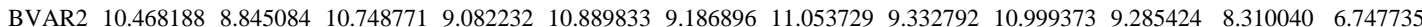

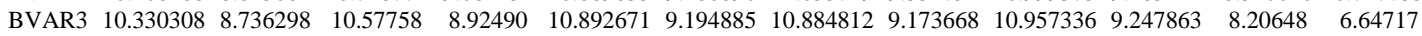

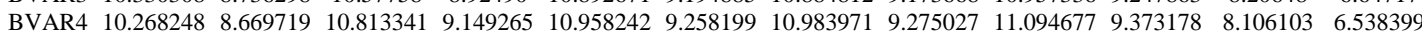




\section{Continued}

\begin{tabular}{|c|c|c|c|c|c|c|c|c|c|}
\hline \multirow{3}{*}{$\begin{array}{c}\text { AUTOCO } \\
\text { LEVELS }(\delta)\end{array}$} & \multirow{3}{*}{ Models } & \multicolumn{8}{|c|}{ COLLINEARITY $(\rho)$} \\
\hline & & \multicolumn{2}{|c|}{0.85} & \multicolumn{2}{|c|}{0.9} & \multicolumn{2}{|c|}{0.95} & \multicolumn{2}{|c|}{0.99} \\
\hline & & RMSE & MAE & RMSE & MAE & RMSE & MAE & RMSE & MAE \\
\hline-0.99 & $\begin{array}{l}\text { VAR(2) } \\
\text { BVAR1 } \\
\text { BVAR2 } \\
\text { BVAR3 } \\
\text { BVAR4 }\end{array}$ & $\begin{array}{c}12.292872 \\
9.940012 \\
9.860353 \\
9.879126 \\
9.872221\end{array}$ & $\begin{array}{l}9.019249 \\
7.615291 \\
7.473166 \\
7.456796 \\
7.397829\end{array}$ & $\begin{array}{c}12.328465 \\
9.695786 \\
9.645700 \\
9.615907 \\
9.693887\end{array}$ & $\begin{array}{l}8.583864 \\
7.151369 \\
7.013643 \\
6.996396 \\
6.985854\end{array}$ & $\begin{array}{c}11.88425 \\
10.003641 \\
9.984819 \\
10.060146 \\
10.175596\end{array}$ & $\begin{array}{c}9.30928 \\
7.811151 \\
7.776508 \\
7.804474 \\
7.888045\end{array}$ & $\begin{array}{c}14.38993 \\
11.401214 \\
11.433606 \\
11.712333 \\
11.771431\end{array}$ & $\begin{array}{l}12.04130 \\
9.578301 \\
9.600694 \\
9.807139 \\
9.858685\end{array}$ \\
\hline-0.95 & $\begin{array}{l}\text { VAR(2) } \\
\text { BVAR1 } \\
\text { BVAR2 } \\
\text { BVAR3 } \\
\text { BVAR4 }\end{array}$ & $\begin{array}{l}4.675627 \\
4.751472 \\
4.575137 \\
4.618396 \\
4.526623\end{array}$ & $\begin{array}{l}3.433047 \\
3.753760 \\
3.503607 \\
3.533003 \\
3.390034\end{array}$ & $\begin{array}{l}4.591227 \\
4.601855 \\
4.451270 \\
4.506708 \\
4.438774\end{array}$ & $\begin{array}{l}3.294811 \\
3.563108 \\
3.330402 \\
3.375573 \\
3.251366\end{array}$ & $\begin{array}{l}4.834659 \\
4.676203 \\
4.558022 \\
4.647846 \\
4.627821\end{array}$ & $\begin{array}{l}3.684773 \\
3.631709 \\
3.496429 \\
3.573142 \\
3.530552\end{array}$ & $\begin{array}{l}5.489967 \\
5.166012 \\
5.160168 \\
5.216748 \\
5.220785\end{array}$ & $\begin{array}{l}4.457119 \\
4.171296 \\
4.168281 \\
4.211559 \\
4.218392\end{array}$ \\
\hline-0.9 & $\begin{array}{l}\text { VAR(2) } \\
\text { BVAR1 } \\
\text { BVAR2 } \\
\text { BVAR3 } \\
\text { BVAR4 }\end{array}$ & $\begin{array}{l}3.122088 \\
3.504443 \\
3.260930 \\
3.275876 \\
3.152633\end{array}$ & $\begin{array}{l}2.313956 \\
2.878414 \\
2.575507 \\
2.585727 \\
2.410320\end{array}$ & $\begin{array}{l}3.083480 \\
3.359757 \\
3.162962 \\
3.191852 \\
3.084599\end{array}$ & $\begin{array}{l}2.249458 \\
2.713640 \\
2.451965 \\
2.476510 \\
2.322219\end{array}$ & $\begin{array}{l}3.197895 \\
3.315275 \\
3.202590 \\
3.228760 \\
3.178185\end{array}$ & $\begin{array}{l}2.428867 \\
2.621622 \\
2.483038 \\
2.507757 \\
2.438380\end{array}$ & $\begin{array}{l}3.603086 \\
3.570148 \\
3.535835 \\
3.571913 \\
3.540043\end{array}$ & $\begin{array}{l}2.879401 \\
2.859518 \\
2.829658 \\
2.857418 \\
2.829462\end{array}$ \\
\hline-0.85 & $\begin{array}{l}\text { VAR(2) } \\
\text { BVAR1 } \\
\text { BVAR2 } \\
\text { BVAR3 } \\
\text { BVAR4 }\end{array}$ & $\begin{array}{l}2.477548 \\
3.006656 \\
2.727614 \\
2.734891 \\
2.574753\end{array}$ & $\begin{array}{l}1.852433 \\
2.517774 \\
2.198541 \\
2.202784 \\
2.002080\end{array}$ & $\begin{array}{l}2.451333 \\
2.848012 \\
2.619965 \\
2.644389 \\
2.515712\end{array}$ & $\begin{array}{l}1.812685 \\
2.350770 \\
2.076338 \\
2.098645 \\
1.929908\end{array}$ & $\begin{array}{l}2.543358 \\
2.767444 \\
2.616711 \\
2.656329 \\
2.570926\end{array}$ & $\begin{array}{l}1.936024 \\
2.215490 \\
2.048485 \\
2.083624 \\
1.985405\end{array}$ & $\begin{array}{l}2.841108 \\
2.872582 \\
2.821017 \\
2.849932 \\
2.823762\end{array}$ & $\begin{array}{l}2.255355 \\
2.290326 \\
2.246513 \\
2.268526 \\
2.245788\end{array}$ \\
\hline-0.8 & $\begin{array}{l}\text { VAR(2) } \\
\text { BVAR1 } \\
\text { BVAR2 } \\
\text { BVAR3 } \\
\text { BVAR4 }\end{array}$ & $\begin{array}{l}2.125443 \\
2.739682 \\
2.425755 \\
2.440397 \\
2.250814\end{array}$ & $\begin{array}{l}1.598810 \\
2.311585 \\
1.978843 \\
1.988378 \\
1.769048\end{array}$ & $\begin{array}{l}2.107579 \\
2.575504 \\
2.326747 \\
2.347586 \\
2.200609\end{array}$ & $\begin{array}{l}1.573870 \\
2.146472 \\
1.866837 \\
1.886717 \\
1.708180\end{array}$ & $\begin{array}{c}2.17960 \\
2.449498 \\
2.296983 \\
2.328026 \\
2.227605\end{array}$ & $\begin{array}{c}1.66439 \\
1.975380 \\
1.809410 \\
1.838238 \\
1.728900\end{array}$ & $\begin{array}{l}2.414951 \\
2.481309 \\
2.419762 \\
2.457924 \\
2.411302\end{array}$ & $\begin{array}{l}1.907861 \\
1.969979 \\
1.918207 \\
1.948747 \\
1.908411\end{array}$ \\
\hline 0.8 & $\begin{array}{l}\text { VAR(2) } \\
\text { BVAR1 } \\
\text { BVAR2 } \\
\text { BVAR3 } \\
\text { BVAR4 }\end{array}$ & $\begin{array}{l}2.236399 \\
2.627007 \\
2.452804 \\
2.501397 \\
2.357946\end{array}$ & $\begin{array}{l}1.754781 \\
2.120110 \\
1.957960 \\
2.007570 \\
1.871715\end{array}$ & $\begin{array}{l}2.299736 \\
2.599947 \\
2.461771 \\
2.504288 \\
2.388347\end{array}$ & $\begin{array}{l}1.814077 \\
2.083388 \\
1.957306 \\
2.000493 \\
1.895858\end{array}$ & $\begin{array}{l}2.379149 \\
2.588574 \\
2.486751 \\
2.509820 \\
2.437277\end{array}$ & $\begin{array}{l}1.884599 \\
2.062693 \\
1.973808 \\
1.997461 \\
1.934261\end{array}$ & $\begin{array}{l}2.487192 \\
2.620266 \\
2.548413 \\
2.550920 \\
2.507633\end{array}$ & $\begin{array}{l}1.974898 \\
2.084377 \\
2.023260 \\
2.026514 \\
1.990963\end{array}$ \\
\hline 0.85 & $\begin{array}{l}\text { VAR(2) } \\
\text { BVAR1 } \\
\text { BVAR2 } \\
\text { BVAR3 } \\
\text { BVAR4 }\end{array}$ & $\begin{array}{l}2.531841 \\
2.894148 \\
2.732076 \\
2.772147 \\
2.638373\end{array}$ & $\begin{array}{l}1.992353 \\
2.332085 \\
2.181343 \\
2.222319 \\
2.096798\end{array}$ & $\begin{array}{l}2.608348 \\
2.884802 \\
2.756491 \\
2.787752 \\
2.683887\end{array}$ & $\begin{array}{l}2.064005 \\
2.310809 \\
2.196467 \\
2.229991 \\
2.135190\end{array}$ & $\begin{array}{l}2.703969 \\
2.903956 \\
2.801762 \\
2.816011 \\
2.747752\end{array}$ & $\begin{array}{l}2.151654 \\
2.318829 \\
2.231792 \\
2.246924 \\
2.188503\end{array}$ & $\begin{array}{l}2.828153 \\
2.952580 \\
2.880183 \\
2.886455 \\
2.846163\end{array}$ & $\begin{array}{l}2.254426 \\
2.357421 \\
2.296912 \\
2.303569 \\
2.270006\end{array}$ \\
\hline 0.9 & $\begin{array}{l}\text { VAR(2) } \\
\text { BVAR1 } \\
\text { BVAR2 } \\
\text { BVAR3 } \\
\text { BVAR4 }\end{array}$ & $\begin{array}{l}3.052437 \\
3.363117 \\
3.218510 \\
3.237278 \\
3.121272\end{array}$ & $\begin{array}{l}2.409829 \\
2.704008 \\
2.569185 \\
2.594169 \\
2.483805\end{array}$ & $\begin{array}{l}3.142645 \\
3.380101 \\
3.262358 \\
3.282231 \\
3.182059\end{array}$ & $\begin{array}{l}2.499225 \\
2.710087 \\
2.605622 \\
2.629490 \\
2.539302\end{array}$ & $\begin{array}{l}3.270464 \\
3.438881 \\
3.345030 \\
3.341460 \\
3.284477\end{array}$ & $\begin{array}{l}2.616470 \\
2.756910 \\
2.676726 \\
2.679199 \\
2.628086\end{array}$ & $\begin{array}{l}3.437442 \\
3.522892 \\
3.468972 \\
3.445365 \\
3.406939\end{array}$ & $\begin{array}{l}2.760244 \\
2.827787 \\
2.781787 \\
2.764382 \\
2.732175\end{array}$ \\
\hline 0.95 & $\begin{array}{l}\text { VAR(2) } \\
\text { BVAR1 } \\
\text { BVAR2 } \\
\text { BVAR3 } \\
\text { BVAR4 }\end{array}$ & $\begin{array}{l}4.365492 \\
4.461304 \\
4.344079 \\
4.299452 \\
4.174909\end{array}$ & $\begin{array}{l}3.471970 \\
3.588159 \\
3.482217 \\
3.450690 \\
3.334882\end{array}$ & $\begin{array}{l}4.480920 \\
4.523729 \\
4.421030 \\
4.383871 \\
4.293484\end{array}$ & $\begin{array}{l}3.607913 \\
3.646270 \\
3.556775 \\
3.527764 \\
3.447778\end{array}$ & $\begin{array}{l}4.766742 \\
4.664817 \\
4.553524 \\
4.515636 \\
4.448287\end{array}$ & $\begin{array}{l}3.856211 \\
3.771176 \\
3.676655 \\
3.648935 \\
3.593866\end{array}$ & $\begin{array}{l}4.938933 \\
4.839329 \\
4.746046 \\
4.716871 \\
4.653130\end{array}$ & $\begin{array}{l}4.029858 \\
3.923619 \\
3.847426 \\
3.821928 \\
3.768907\end{array}$ \\
\hline 0.99 & $\begin{array}{l}\text { VAR(2) } \\
\text { BVAR1 } \\
\text { BVAR2 } \\
\text { BVAR3 } \\
\text { BVAR4 }\end{array}$ & $\begin{array}{c}11.981223 \\
8.696257 \\
8.479994 \\
8.475901 \\
8.230192\end{array}$ & $\begin{array}{l}9.694264 \\
7.160028 \\
6.960884 \\
6.963413 \\
6.741359\end{array}$ & $\begin{array}{l}13.79347 \\
8.932930 \\
8.680591 \\
8.714584 \\
8.491010\end{array}$ & $\begin{array}{l}11.11085 \\
7.420823 \\
7.202460 \\
7.230251 \\
7.038407\end{array}$ & $\begin{array}{l}14.74004 \\
9.202919 \\
9.164582 \\
9.034855 \\
8.825268\end{array}$ & $\begin{array}{l}11.94566 \\
7.715310 \\
7.676524 \\
7.575599 \\
7.392270\end{array}$ & $\begin{array}{l}13.85595 \\
9.746341 \\
9.602264 \\
9.403324 \\
9.410795\end{array}$ & $\begin{array}{l}11.80282 \\
8.217796 \\
8.101851 \\
7.920777 \\
7.930210\end{array}$ \\
\hline
\end{tabular}




\section{Appendix B}

Table B1. The ranks of the performances of the model for $T=8$.

\begin{tabular}{|c|c|c|c|c|c|c|c|c|c|c|c|c|c|}
\hline \multirow{3}{*}{$\begin{array}{c}T=8 \\
\text { AUTOCO } \\
\text { LEVELS }(\delta)\end{array}$} & \multirow{3}{*}{ Models } & \multicolumn{12}{|c|}{ RANK COLLINERITY $(\rho)$} \\
\hline & & \multicolumn{2}{|c|}{-0.99} & \multicolumn{2}{|c|}{-0.95} & \multicolumn{2}{|c|}{-0.9} & \multicolumn{2}{|c|}{-0.85} & \multicolumn{2}{|c|}{-0.8} & \multicolumn{2}{|c|}{0.8} \\
\hline & & RMSE & MAE & RMSE & MAE & RMSE & MAE & RMSE & MAE & RMSE & MAE & RMSE & MAE \\
\hline \multirow{5}{*}{-0.99} & VAR(2) & N/A & N/A & N/A & N/A & N/A & N/A & N/A & N/A & N/A & N/A & N/A & N/A \\
\hline & BVAR1 & 4 & 4 & 4 & 4 & 4 & 4 & 4 & 4 & 4 & 4 & 4 & 3 \\
\hline & BVAR2 & 3 & 3 & 3 & 3 & 3 & 3 & 3 & 3 & 3 & 3 & 3 & 1 \\
\hline & BVAR3 & 2 & 2 & 2 & 2 & 2 & 2 & 2 & 2 & 2 & 2 & 2 & 2 \\
\hline & BVAR4 & 1 & 1 & 1 & 1 & 1 & 1 & 1 & 1 & 1 & 1 & 1 & 4 \\
\hline \multirow{5}{*}{-0.95} & VAR(2) & N/A & N/A & N/A & N/A & N/A & N/A & N/A & N/A & N/A & N/A & N/A & N/A \\
\hline & BVAR1 & 4 & 4 & 4 & 4 & 4 & 4 & 4 & 4 & 4 & 4 & 4 & 2 \\
\hline & BVAR2 & 3 & 3 & 3 & 3 & 3 & 3 & 3 & 3 & 3 & 3 & 3 & 1 \\
\hline & BVAR3 & 2 & 2 & 2 & 2 & 2 & 2 & 2 & 2 & 2 & 2 & 1 & 3 \\
\hline & BVAR4 & 1 & 1 & 1 & 1 & 1 & 1 & 1 & 1 & 1 & 1 & 2 & 4 \\
\hline \multirow{5}{*}{-0.9} & VAR(2) & N/A & N/A & N/A & N/A & N/A & N/A & N/A & N/A & N/A & N/A & N/A & N/A \\
\hline & BVAR1 & 3 & 3 & 4 & 4 & 4 & 4 & 4 & 4 & 4 & 4 & 2 & 1 \\
\hline & BVAR2 & 4 & 4 & 3 & 3 & 3 & 3 & 3 & 3 & 3 & 3 & 1 & 2 \\
\hline & BVAR3 & 2 & 2 & 2 & 2 & 2 & 2 & 2 & 2 & 2 & 2 & 3 & 3 \\
\hline & BVAR4 & 1 & 1 & 1 & 1 & 1 & 1 & 1 & 1 & 1 & 1 & 4 & 4 \\
\hline \multirow{5}{*}{-0.85} & VAR(2) & N/A & N/A & N/A & N/A & N/A & N/A & N/A & N/A & N/A & N/A & N/A & N/A \\
\hline & BVAR1 & 4 & 4 & 4 & 3 & 4 & 4 & 4 & 4 & 4 & 4 & 1 & 1 \\
\hline & BVAR2 & 3 & 3 & 3 & 4 & 3 & 3 & 3 & 3 & 3 & 3 & 2 & 2 \\
\hline & BVAR3 & 2 & 2 & 2 & 2 & 2 & 2 & 2 & 2 & 2 & 2 & 3 & 3 \\
\hline & BVAR4 & 1 & 1 & 1 & 1 & 1 & 1 & 1 & 1 & 1 & 1 & 4 & 4 \\
\hline \multirow{5}{*}{-0.8} & VAR(2) & N/A & N/A & N/A & N/A & N/A & N/A & N/A & N/A & N/A & N/A & N/A & N/A \\
\hline & BVAR1 & 3 & 3 & 4 & 4 & 4 & 4 & 4 & 4 & 4 & 4 & 1 & 1 \\
\hline & BVAR2 & 4 & 4 & 3 & 3 & 3 & 3 & 3 & 3 & 3 & 3 & 2 & 2 \\
\hline & BVAR3 & 1 & 1 & 2 & 2 & 2 & 2 & 2 & 2 & 2 & 2 & 3 & 3 \\
\hline & BVAR4 & 2 & 2 & 1 & 1 & 1 & 1 & 1 & 1 & 1 & 1 & 4 & 4 \\
\hline \multirow{5}{*}{0.8} & VAR(2) & N/A & N/A & N/A & N/A & N/A & N/A & N/A & N/A & N/A & N/A & N/A & N/A \\
\hline & BVAR1 & 1 & 1 & 3 & 2 & 4 & 3 & 3 & 3 & 4 & 3 & 1 & 1 \\
\hline & BVAR2 & 3 & 2 & 4 & 4 & 3 & 4 & 4 & 4 & 3 & 4 & 2 & 2 \\
\hline & BVAR3 & 2 & 3 & 1 & 1 & 2 & 2 & 2 & 2 & 2 & 1 & 3 & 3 \\
\hline & BVAR4 & 4 & 4 & 2 & 3 & 1 & 1 & 1 & 1 & 1 & 2 & 4 & 4 \\
\hline \multirow{5}{*}{0.85} & VAR(2) & N/A & N/A & N/A & N/A & N/A & N/A & N/A & N/A & N/A & N/A & N/A & N/A \\
\hline & BVAR1 & 3 & 1 & 3 & 3 & 4 & 3 & 4 & 3 & 3 & 2 & 1 & 1 \\
\hline & BVAR2 & 2 & 2 & 4 & 4 & 3 & 4 & 3 & 4 & 4 & 4 & 2 & 2 \\
\hline & BVAR3 & 1 & 3 & 1 & 1 & 2 & 2 & 2 & 1 & 1 & 1 & 3 & 3 \\
\hline & BVAR4 & 4 & 4 & 2 & 2 & 1 & 1 & 1 & 2 & 2 & 3 & 4 & 4 \\
\hline & VAR(2) & N/A & N/A & N/A & N/A & N/A & N/A & N/A & N/A & N/A & N/A & N/A & N/A \\
\hline & BVAR1 & 1 & 1 & 3 & 2 & 4 & 4 & 3 & 3 & 3 & 3 & 1 & 1 \\
\hline 0.9 & BVAR2 & 2 & 2 & 4 & 4 & 3 & 3 & 4 & 4 & 4 & 4 & 2 & 2 \\
\hline & BVAR3 & 3 & 3 & 2 & 3 & 2 & 2 & 2 & 2 & 2 & 1 & 3 & 3 \\
\hline & BVAR4 & 4 & 4 & 1 & 1 & 1 & 1 & 1 & 1 & 1 & 2 & 4 & 4 \\
\hline & VAR(2) & N/A & N/A & N/A & N/A & N/A & N/A & N/A & N/A & N/A & N/A & N/A & N/A \\
\hline & BVAR1 & 2 & 1 & 4 & 4 & 3 & 3 & 3 & 3 & 3 & 3 & 1 & 1 \\
\hline 0.95 & BVAR2 & 3 & 2 & 3 & 2 & 4 & 4 & 4 & 4 & 4 & 4 & 2 & 2 \\
\hline & BVAR3 & 1 & 3 & 1 & 1 & 2 & 2 & 2 & 2 & 2 & 2 & 3 & 3 \\
\hline & BVAR4 & 4 & 4 & 2 & 3 & 1 & 1 & 1 & 1 & 1 & 1 & 4 & 4 \\
\hline & VAR(2) & N/A & N/A & N/A & N/A & N/A & N/A & N/A & N/A & N/A & N/A & N/A & N/A \\
\hline & BVAR1 & 1 & 1 & 4 & 4 & 4 & 3 & 4 & 2 & 3 & 3 & 1 & 1 \\
\hline 0.99 & BVAR2 & 3 & 2 & 3 & 3 & 3 & 4 & 3 & 4 & 4 & 4 & 2 & 2 \\
\hline & BVAR3 & 2 & 3 & 2 & 1 & 2 & 1 & 2 & 1 & 2 & 2 & 3 & 3 \\
\hline & BVAR4 & 4 & 4 & 1 & 2 & 1 & 2 & 1 & 3 & 1 & 1 & 4 & 4 \\
\hline
\end{tabular}




\section{Continued}

\begin{tabular}{|c|c|c|c|c|c|c|c|c|c|}
\hline \multirow{3}{*}{$\begin{array}{c}T=8 \\
\text { AUTOCO } \\
\text { LEVELS }(\delta)\end{array}$} & \multirow{3}{*}{ Models } & \multicolumn{8}{|c|}{ COLLINEARITY $(\rho)$} \\
\hline & & \multicolumn{2}{|c|}{0.85} & \multicolumn{2}{|c|}{0.9} & \multicolumn{2}{|c|}{0.95} & \multicolumn{2}{|c|}{0.99} \\
\hline & & RMSE & MAE & RMSE & MAE & RMSE & MAE & RMSE & MAE \\
\hline \multirow{5}{*}{-0.99} & VAR(2) & N/A & N/A & N/A & N/A & N/A & N/A & N/A & N/A \\
\hline & BVAR1 & 3 & 1 & 4 & 1 & 4 & 1 & 4 & 1 \\
\hline & BVAR2 & 4 & 2 & 3 & 2 & 3 & 2 & 3 & 4 \\
\hline & BVAR3 & 2 & 4 & 1 & 3 & 2 & 3 & 2 & 3 \\
\hline & BVAR4 & 1 & 3 & 2 & 4 & 1 & 4 & 1 & 2 \\
\hline \multirow{5}{*}{-0.95} & VAR(2) & N/A & N/A & N/A & N/A & N/A & N/A & N/A & N/A \\
\hline & BVAR1 & 3 & 1 & 1 & 1 & 1 & 1 & 4 & 1 \\
\hline & BVAR2 & 4 & 2 & 2 & 2 & 2 & 2 & 3 & 2 \\
\hline & BVAR3 & 1 & 3 & 3 & 3 & 3 & 3 & 1 & 3 \\
\hline & BVAR4 & 2 & 4 & 4 & 4 & 4 & 4 & 2 & 4 \\
\hline \multirow{5}{*}{-0.9} & VAR(2) & N/A & N/A & N/A & N/A & N/A & N/A & N/A & N/A \\
\hline & BVAR1 & 1 & 1 & 2 & 1 & 1 & 1 & 3 & 1 \\
\hline & BVAR2 & 2 & 2 & 1 & 2 & 2 & 2 & 2 & 2 \\
\hline & BVAR3 & 3 & 3 & 3 & 3 & 3 & 3 & 1 & 3 \\
\hline & BVAR4 & 4 & 4 & 4 & 4 & 4 & 4 & 4 & 4 \\
\hline \multirow{5}{*}{-0.85} & VAR(2) & N/A & N/A & N/A & N/A & N/A & N/A & N/A & N/A \\
\hline & BVAR1 & 1 & 1 & 1 & 1 & 2 & 1 & 1 & 1 \\
\hline & BVAR2 & 2 & 2 & 2 & 2 & 1 & 2 & 2 & 2 \\
\hline & BVAR3 & 3 & 3 & 3 & 3 & 3 & 3 & 3 & 3 \\
\hline & BVAR4 & 4 & 4 & 4 & 4 & 4 & 4 & 4 & 4 \\
\hline \multirow{5}{*}{-0.8} & VAR(2) & N/A & N/A & N/A & N/A & N/A & N/A & N/A & N/A \\
\hline & BVAR1 & 1 & 1 & 1 & 1 & 1 & 1 & 2 & 1 \\
\hline & BVAR2 & 2 & 2 & 2 & 2 & 2 & 2 & 1 & 2 \\
\hline & BVAR3 & 3 & 3 & 3 & 3 & 3 & 3 & 3 & 3 \\
\hline & BVAR4 & 4 & 4 & 4 & 4 & 4 & 4 & 4 & 4 \\
\hline \multirow{5}{*}{0.8} & VAR(2) & N/A & N/A & N/A & N/A & N/A & N/A & N/A & N/A \\
\hline & BVAR1 & 1 & 1 & 1 & 1 & 1 & 1 & 1 & 1 \\
\hline & BVAR2 & 2 & 2 & 2 & 2 & 2 & 2 & 2 & 2 \\
\hline & BVAR3 & 3 & 3 & 3 & 3 & 3 & 3 & 3 & 3 \\
\hline & BVAR4 & 4 & 4 & 4 & 4 & 4 & 4 & 4 & 4 \\
\hline \multirow{5}{*}{0.85} & VAR(2) & N/A & N/A & N/A & N/A & N/A & N/A & N/A & N/A \\
\hline & BVAR1 & 1 & 1 & 1 & 1 & 1 & 1 & 1 & 1 \\
\hline & BVAR2 & 2 & 2 & 2 & 2 & 2 & 2 & 2 & 2 \\
\hline & BVAR3 & 3 & 3 & 3 & 3 & 3 & 3 & 3 & 3 \\
\hline & BVAR4 & 4 & 4 & 4 & 4 & 4 & 4 & 4 & 4 \\
\hline \multirow{5}{*}{0.9} & VAR(2) & N/A & N/A & N/A & N/A & N/A & N/A & N/A & N/A \\
\hline & BVAR1 & 1 & 1 & 2 & 1 & 2 & 1 & 1 & 1 \\
\hline & BVAR2 & 2 & 2 & 1 & 2 & 1 & 2 & 2 & 2 \\
\hline & BVAR3 & 3 & 3 & 3 & 3 & 3 & 3 & 3 & 3 \\
\hline & BVAR4 & 4 & 4 & 4 & 4 & 4 & 4 & 4 & 4 \\
\hline \multirow{5}{*}{0.95} & VAR(2) & N/A & N/A & N/A & N/A & N/A & N/A & N/A & N/A \\
\hline & BVAR1 & 1 & 1 & 1 & 1 & 1 & 1 & 1 & 1 \\
\hline & BVAR2 & 2 & 2 & 2 & 2 & 2 & 2 & 2 & 2 \\
\hline & BVAR3 & 3 & 3 & 3 & 3 & 3 & 3 & 3 & 3 \\
\hline & BVAR4 & 4 & 4 & 4 & 4 & 4 & 4 & 4 & 4 \\
\hline \multirow{5}{*}{0.99} & VAR(2) & N/A & N/A & N/A & N/A & N/A & N/A & N/A & N/A \\
\hline & BVAR1 & 2 & 1 & 1 & 1 & 1 & 1 & 1 & 1 \\
\hline & BVAR2 & 1 & 2 & 2 & 2 & 2 & 2 & 2 & 2 \\
\hline & BVAR3 & 3 & 3 & 3 & 3 & 3 & 3 & 3 & 3 \\
\hline & BVAR4 & 4 & 4 & 4 & 4 & 4 & 4 & 4 & 4 \\
\hline
\end{tabular}


Table B2. The ranks of the performances of the model for $T=16$.

\begin{tabular}{|c|c|c|c|c|c|c|c|c|c|c|c|c|c|}
\hline \multirow{3}{*}{$\begin{array}{c}T=16 \\
\text { AUTOCO } \\
\text { LEVELS }(\delta)\end{array}$} & \multirow{3}{*}{ Models } & \multicolumn{12}{|c|}{ COLLINERITY $(\rho)$} \\
\hline & & \multicolumn{2}{|c|}{-0.99} & \multicolumn{2}{|c|}{-0.95} & \multicolumn{2}{|c|}{-0.9} & \multicolumn{2}{|c|}{-0.85} & \multicolumn{2}{|c|}{-0.8} & \multicolumn{2}{|c|}{0.8} \\
\hline & & RMSE & MAE & RMSE & MAE & RMSE & MAE & RMSE & MAE & RMSE & MAE & RMSE & MAE \\
\hline \multirow{5}{*}{-0.99} & VAR(2) & 5 & 5 & 5 & 5 & 5 & 5 & 5 & 5 & 5 & 5 & 5 & 5 \\
\hline & BVAR1 & 4 & 4 & 4 & 4 & 4 & 4 & 4 & 4 & 4 & 4 & 4 & 4 \\
\hline & BVAR2 & 3 & 3 & 3 & 3 & 3 & 3 & 3 & 3 & 3 & 3 & 3 & 3 \\
\hline & BVAR3 & 2 & 2 & 2 & 2 & 2 & 2 & 2 & 2 & 2 & 2 & 2 & 2 \\
\hline & BVAR4 & 1 & 1 & 1 & 1 & 1 & 1 & 1 & 1 & 1 & 1 & 1 & 1 \\
\hline \multirow{5}{*}{-0.95} & VAR(2) & 5 & 5 & 5 & 5 & 5 & 5 & 5 & 5 & 5 & 5 & 5 & 5 \\
\hline & BVAR1 & 4 & 4 & 4 & 4 & 4 & 4 & 4 & 4 & 4 & 4 & 4 & 4 \\
\hline & BVAR2 & 3 & 3 & 3 & 3 & 3 & 3 & 3 & 3 & 3 & 3 & 3 & 3 \\
\hline & BVAR3 & 2 & 2 & 2 & 2 & 2 & 2 & 2 & 2 & 2 & 2 & 1 & 1 \\
\hline & BVAR4 & 1 & 1 & 1 & 1 & 1 & 1 & 1 & 1 & 1 & 1 & 2 & 2 \\
\hline \multirow{5}{*}{-0.9} & VAR(2) & 5 & 5 & 5 & 5 & 5 & 5 & 5 & 5 & 5 & 5 & 5 & 5 \\
\hline & BVAR1 & 4 & 4 & 4 & 4 & 4 & 4 & 4 & 4 & 4 & 4 & 4 & 3 \\
\hline & BVAR2 & 3 & 3 & 3 & 3 & 3 & 3 & 3 & 3 & 3 & 3 & 3 & 1 \\
\hline & BVAR3 & 2 & 2 & 2 & 2 & 2 & 2 & 2 & 2 & 2 & 2 & 1 & 2 \\
\hline & BVAR4 & 1 & 1 & 1 & 1 & 1 & 1 & 1 & 1 & 1 & 1 & 2 & 4 \\
\hline \multirow{5}{*}{-0.85} & VAR(2) & 5 & 5 & 5 & 5 & 5 & 5 & 5 & 5 & 5 & 5 & 5 & 5 \\
\hline & BVAR1 & 4 & 4 & 4 & 4 & 4 & 4 & 4 & 4 & 4 & 4 & 4 & 2 \\
\hline & BVAR2 & 3 & 3 & 3 & 3 & 3 & 3 & 3 & 3 & 3 & 3 & 1 & 1 \\
\hline & BVAR3 & 2 & 2 & 2 & 2 & 2 & 2 & 2 & 2 & 2 & 2 & 3 & 4 \\
\hline & BVAR4 & 1 & 1 & 1 & 1 & 1 & 1 & 1 & 1 & 1 & 1 & 2 & 3 \\
\hline \multirow{5}{*}{-0.8} & VAR(2) & 5 & 5 & 5 & 5 & 5 & 5 & 5 & 5 & 5 & 5 & 5 & 3 \\
\hline & BVAR1 & 4 & 4 & 4 & 4 & 4 & 4 & 4 & 4 & 4 & 4 & 1 & 1 \\
\hline & BVAR2 & 3 & 3 & 3 & 3 & 3 & 3 & 3 & 3 & 3 & 3 & 2 & 2 \\
\hline & BVAR3 & 2 & 2 & 2 & 2 & 2 & 2 & 2 & 2 & 2 & 2 & 4 & 4 \\
\hline & BVAR4 & 1 & 1 & 1 & 1 & 1 & 1 & 1 & 1 & 1 & 1 & 3 & 5 \\
\hline \multirow{5}{*}{0.8} & VAR(2) & 5 & 5 & 5 & 5 & 5 & 5 & 5 & 5 & 5 & 5 & 5 & 5 \\
\hline & BVAR1 & 3 & 3 & 4 & 4 & 3 & 3 & 3 & 3 & 4 & 3 & 1 & 1 \\
\hline & BVAR2 & 4 & 4 & 3 & 3 & 4 & 4 & 4 & 4 & 3 & 4 & 2 & 2 \\
\hline & BVAR3 & 2 & 2 & 2 & 2 & 2 & 2 & 2 & 2 & 2 & 2 & 4 & 4 \\
\hline & BVAR4 & 1 & 1 & 1 & 1 & 1 & 1 & 1 & 1 & 1 & 1 & 3 & 3 \\
\hline \multirow{5}{*}{0.85} & VAR(2) & 5 & 5 & 5 & 5 & 5 & 5 & 5 & 5 & 5 & 5 & 5 & 5 \\
\hline & BVAR1 & 3 & 3 & 4 & 4 & 4 & 4 & 4 & 4 & 3 & 3 & 2 & 2 \\
\hline & BVAR2 & 4 & 4 & 3 & 3 & 3 & 3 & 3 & 3 & 4 & 4 & 1 & 1 \\
\hline & BVAR3 & 2 & 2 & 2 & 2 & 2 & 2 & 2 & 2 & 2 & 2 & 4 & 4 \\
\hline & BVAR4 & 1 & 1 & 1 & 1 & 1 & 1 & 1 & 1 & 1 & 1 & 3 & 3 \\
\hline \multirow{5}{*}{0.9} & VAR(2) & 5 & 5 & 5 & 5 & 5 & 5 & 5 & 5 & 5 & 5 & 5 & 5 \\
\hline & BVAR1 & 4 & 4 & 4 & 4 & 4 & 3 & 4 & 3 & 3 & 3 & 1 & 1 \\
\hline & BVAR2 & 3 & 3 & 3 & 3 & 3 & 4 & 3 & 4 & 4 & 4 & 2 & 2 \\
\hline & BVAR3 & 2 & 2 & 2 & 2 & 2 & 2 & 2 & 2 & 2 & 2 & 4 & 4 \\
\hline & BVAR4 & 1 & 1 & 1 & 1 & 1 & 1 & 1 & 1 & 1 & 1 & 3 & 3 \\
\hline
\end{tabular}


M. O. Adenomon et al.

\section{Continued}

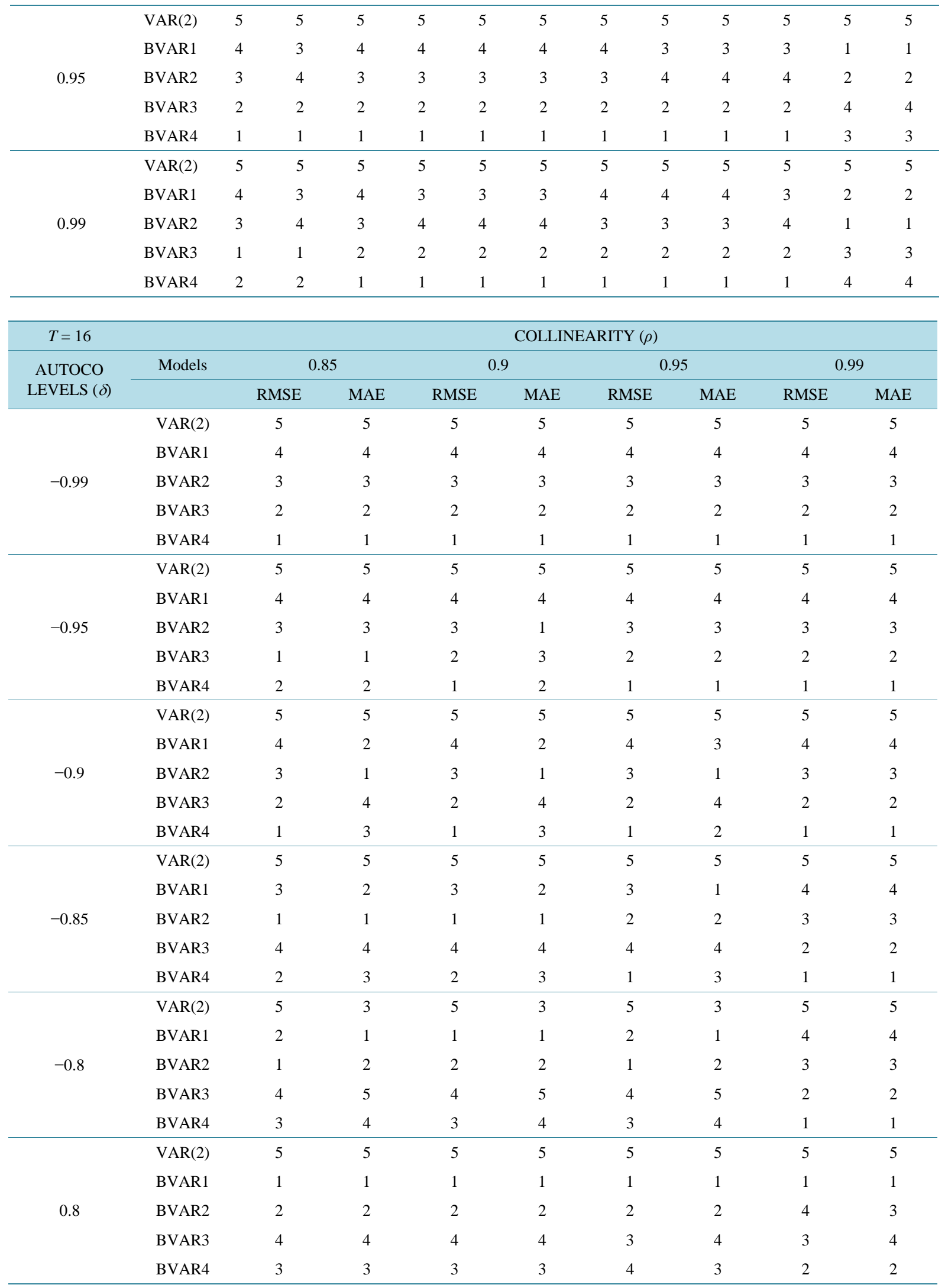




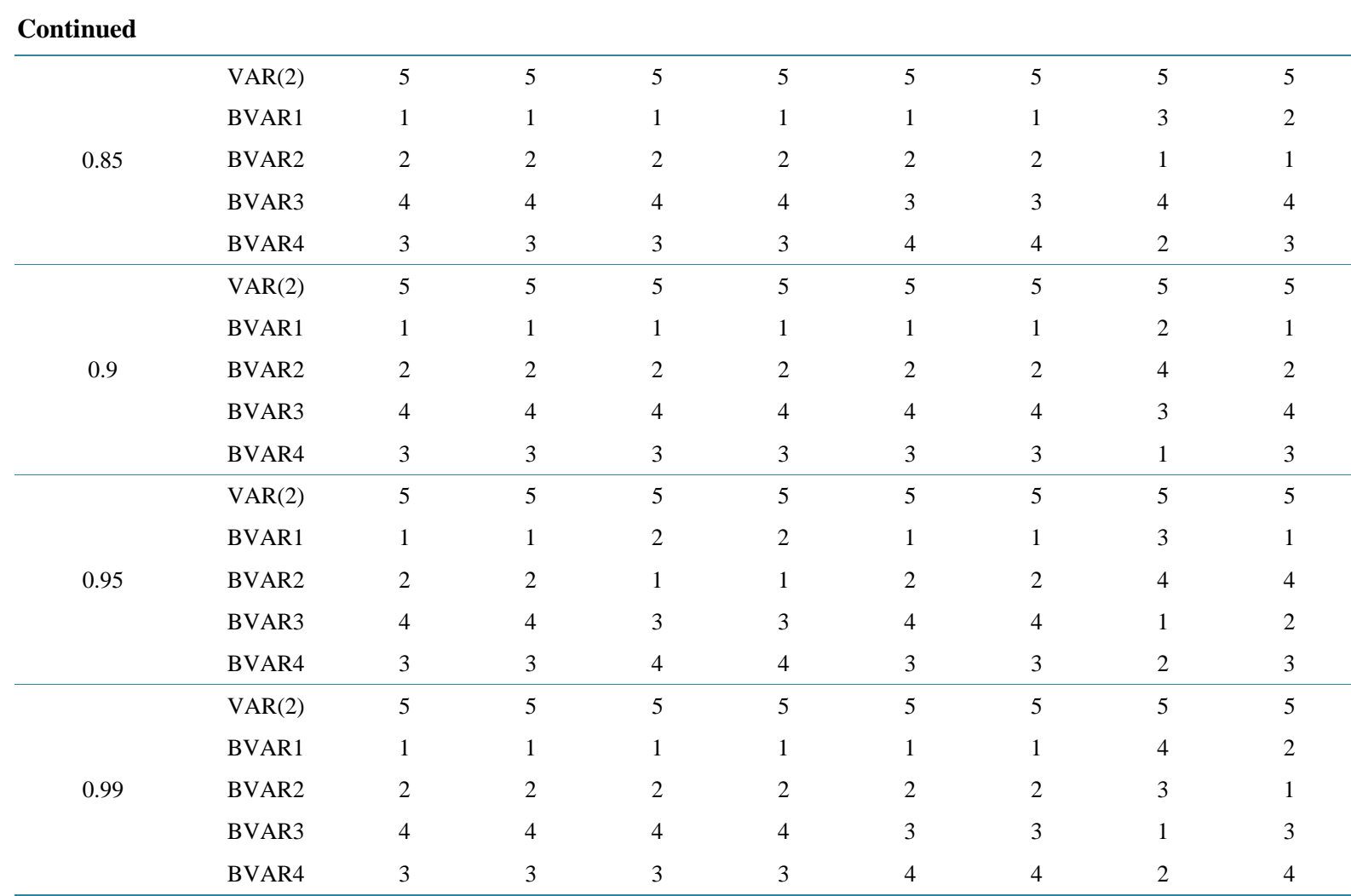

Table B3. The ranks of the performances of the model for $T=32$.

\begin{tabular}{|c|c|c|c|c|c|c|c|c|c|c|c|c|c|}
\hline \multicolumn{2}{|l|}{$T=32$} & \multicolumn{12}{|c|}{ COLLINERITY $(\rho)$} \\
\hline \multirow{2}{*}{$\begin{array}{c}\text { AUTOCO } \\
\text { LEVELS ( })\end{array}$} & \multirow[t]{2}{*}{ Models } & \multicolumn{2}{|c|}{-0.99} & \multicolumn{2}{|c|}{-0.95} & \multicolumn{2}{|c|}{-0.9} & \multicolumn{2}{|c|}{-0.85} & \multicolumn{2}{|c|}{-0.8} & \multicolumn{2}{|c|}{0.8} \\
\hline & & RMSE & MAE & RMSE & MAE & RMSE & MAE & RMSE & MAE & RMSE & MAE & RMSE & MAE \\
\hline \multirow{5}{*}{-0.99} & $\operatorname{VAR}(2)$ & 5 & 5 & 5 & 5 & 5 & 5 & 5 & 5 & 5 & 5 & 5 & 5 \\
\hline & BVAR1 & 4 & 4 & 3 & 3 & 4 & 4 & 4 & 4 & 4 & 4 & 4 & 4 \\
\hline & BVAR2 & 3 & 3 & 4 & 4 & 3 & 3 & 3 & 3 & 3 & 3 & 2 & 3 \\
\hline & BVAR3 & 2 & 2 & 2 & 2 & 2 & 2 & 2 & 2 & 2 & 2 & 3 & 2 \\
\hline & BVAR4 & 1 & 1 & 1 & 1 & 1 & 1 & 1 & 1 & 1 & 1 & 1 & 1 \\
\hline \multirow{5}{*}{-0.95} & VAR(2) & 5 & 5 & 5 & 5 & 5 & 5 & 5 & 5 & 5 & 5 & 5 & 5 \\
\hline & BVAR1 & 4 & 4 & 4 & 4 & 4 & 4 & 4 & 4 & 4 & 4 & 2 & 2 \\
\hline & BVAR2 & 3 & 3 & 3 & 3 & 3 & 3 & 3 & 3 & 3 & 3 & 1 & 1 \\
\hline & BVAR3 & 2 & 2 & 2 & 2 & 1 & 1 & 2 & 2 & 2 & 2 & 4 & 4 \\
\hline & BVAR4 & 1 & 1 & 1 & 1 & 2 & 2 & 1 & 1 & 1 & 1 & 3 & 3 \\
\hline \multirow{5}{*}{-0.9} & VAR(2) & 5 & 5 & 5 & 5 & 5 & 5 & 5 & 5 & 5 & 5 & 5 & 3 \\
\hline & BVAR1 & 4 & 4 & 4 & 4 & 4 & 4 & 4 & 4 & 4 & 4 & 2 & 2 \\
\hline & BVAR2 & 3 & 3 & 3 & 3 & 3 & 3 & 3 & 3 & 3 & 3 & 1 & 1 \\
\hline & BVAR3 & 2 & 2 & 2 & 2 & 2 & 2 & 2 & 2 & 2 & 2 & 4 & 5 \\
\hline & BVAR4 & 1 & 1 & 1 & 1 & 1 & 1 & 1 & 1 & 1 & 1 & 3 & 4 \\
\hline \multirow{5}{*}{-0.85} & VAR(2) & 5 & 5 & 5 & 5 & 5 & 5 & 5 & 5 & 5 & 5 & 3 & 3 \\
\hline & BVAR1 & 4 & 4 & 4 & 4 & 4 & 4 & 3 & 3 & 4 & 4 & 2 & 2 \\
\hline & BVAR2 & 3 & 3 & 3 & 3 & 3 & 3 & 4 & 4 & 3 & 3 & 1 & 1 \\
\hline & BVAR3 & 2 & 1 & 2 & 2 & 2 & 2 & 1 & 1 & 2 & 1 & 5 & 5 \\
\hline & BVAR4 & 1 & 2 & 1 & 1 & 1 & 1 & 2 & 2 & 1 & 2 & 4 & 4 \\
\hline
\end{tabular}


M. O. Adenomon et al.

\section{Continued}

\begin{tabular}{|c|c|c|c|c|c|c|c|c|c|c|c|c|c|}
\hline & VAR(2) & 5 & 5 & 5 & 5 & 5 & 5 & 5 & 5 & 5 & 5 & 1 & 1 \\
\hline & BVAR1 & 4 & 4 & 4 & 4 & 4 & 4 & 4 & 4 & 4 & 4 & 3 & 2 \\
\hline \multirow[t]{5}{*}{-0.8} & BVAR2 & 3 & 3 & 3 & 3 & 3 & 3 & 3 & 3 & 3 & 3 & 2 & 3 \\
\hline & BVAR3 & 2 & 2 & 2 & 2 & 2 & 2 & 2 & 2 & 2 & 2 & 5 & 5 \\
\hline & BVAR4 & 1 & 1 & 1 & 1 & 1 & 1 & 1 & 1 & 1 & 1 & 4 & 4 \\
\hline & VAR(2) & 5 & 5 & 5 & 5 & 5 & 5 & 5 & 5 & 5 & 5 & 1 & 1 \\
\hline & BVAR1 & 4 & 4 & 4 & 4 & 4 & 4 & 4 & 4 & 4 & 4 & 3 & 3 \\
\hline \multirow[t]{5}{*}{0.8} & BVAR2 & 3 & 3 & 3 & 3 & 3 & 3 & 3 & 3 & 3 & 3 & 4 & 4 \\
\hline & BVAR3 & 2 & 2 & 2 & 2 & 2 & 2 & 2 & 2 & 2 & 2 & 5 & 5 \\
\hline & BVAR4 & 1 & 1 & 1 & 1 & 1 & 1 & 1 & 1 & 1 & 1 & 2 & 2 \\
\hline & VAR(2) & 5 & 5 & 5 & 5 & 5 & 5 & 5 & 5 & 5 & 5 & 2 & 1 \\
\hline & BVAR1 & 4 & 4 & 4 & 4 & 4 & 4 & 4 & 4 & 4 & 4 & 3 & 3 \\
\hline \multirow[t]{5}{*}{0.85} & BVAR2 & 3 & 3 & 3 & 3 & 3 & 3 & 3 & 3 & 3 & 3 & 4 & 4 \\
\hline & BVAR3 & 2 & 2 & 2 & 2 & 2 & 2 & 2 & 2 & 2 & 2 & 5 & 5 \\
\hline & BVAR4 & 1 & 1 & 1 & 1 & 1 & 1 & 1 & 1 & 1 & 1 & 1 & 2 \\
\hline & VAR(2) & 5 & 5 & 5 & 5 & 5 & 5 & 5 & 5 & 5 & 5 & 5 & 5 \\
\hline & BVAR1 & 4 & 4 & 4 & 4 & 4 & 4 & 4 & 4 & 4 & 4 & 2 & 2 \\
\hline \multirow[t]{5}{*}{0.9} & BVAR2 & 3 & 3 & 3 & 3 & 3 & 3 & 3 & 3 & 3 & 3 & 3 & 3 \\
\hline & BVAR3 & 2 & 2 & 2 & 2 & 2 & 2 & 2 & 2 & 2 & 2 & 4 & 4 \\
\hline & BVAR4 & 1 & 1 & 1 & 1 & 1 & 1 & 1 & 1 & 1 & 1 & 1 & 1 \\
\hline & VAR(2) & 5 & 5 & 5 & 5 & 5 & 5 & 5 & 5 & 5 & 5 & 5 & 5 \\
\hline & BVAR1 & 4 & 4 & 4 & 4 & 4 & 4 & 4 & 4 & 4 & 4 & 3 & 2 \\
\hline \multirow[t]{5}{*}{0.95} & BVAR2 & 3 & 3 & 3 & 3 & 3 & 3 & 3 & 3 & 3 & 3 & 4 & 3 \\
\hline & BVAR3 & 2 & 2 & 2 & 2 & 2 & 2 & 2 & 2 & 2 & 2 & 2 & 4 \\
\hline & BVAR4 & 1 & 1 & 1 & 1 & 1 & 1 & 1 & 1 & 1 & 1 & 1 & 1 \\
\hline & VAR(2) & 5 & 5 & 5 & 5 & 5 & 5 & 5 & 5 & 5 & 5 & 5 & 5 \\
\hline & BVAR1 & 4 & 3 & 4 & 3 & 3 & 3 & 3 & 3 & 3 & 3 & 3 & 2 \\
\hline \multirow[t]{3}{*}{0.99} & BVAR2 & 3 & 4 & 3 & 4 & 4 & 4 & 4 & 4 & 4 & 4 & 4 & 4 \\
\hline & BVAR3 & 1 & 1 & 2 & 2 & 2 & 2 & 1 & 1 & 1 & 1 & 2 & 3 \\
\hline & BVAR4 & 2 & 2 & 1 & 1 & 1 & 1 & 2 & 2 & 2 & 2 & 1 & 1 \\
\hline
\end{tabular}

\begin{tabular}{|c|c|c|c|c|c|c|c|c|c|}
\hline \multirow{3}{*}{$\begin{array}{c}T=32 \\
\text { AUTOCO } \\
\text { LEVELS }(\delta)\end{array}$} & \multirow{3}{*}{ Models } & \multicolumn{8}{|c|}{ COLLINEARITY $(\rho)$} \\
\hline & & \multicolumn{2}{|c|}{0.85} & \multicolumn{2}{|c|}{0.9} & \multicolumn{2}{|c|}{0.95} & \multicolumn{2}{|c|}{0.99} \\
\hline & & RMSE & MAE & RMSE & MAE & RMSE & MAE & RMSE & MAE \\
\hline \multirow{5}{*}{-0.99} & VAR(2) & 5 & 5 & 5 & 5 & 5 & 5 & 5 & 5 \\
\hline & BVAR1 & 3 & 3 & 3 & 2 & 4 & 4 & 4 & 4 \\
\hline & BVAR2 & 2 & 2 & 2 & 1 & 3 & 3 & 3 & 3 \\
\hline & BVAR3 & 4 & 4 & 4 & 4 & 2 & 2 & 2 & 2 \\
\hline & BVAR4 & 1 & 1 & 1 & 3 & 1 & 1 & 1 & 1 \\
\hline \multirow{5}{*}{-0.95} & VAR(2) & 5 & 5 & 5 & 5 & 5 & 5 & 5 & 5 \\
\hline & BVAR1 & 2 & 2 & 2 & 2 & 3 & 2 & 4 & 4 \\
\hline & BVAR2 & 1 & 1 & 1 & 1 & 1 & 1 & 2 & 2 \\
\hline & BVAR3 & 4 & 4 & 4 & 4 & 4 & 4 & 3 & 3 \\
\hline & BVAR4 & 3 & 3 & 3 & 3 & 2 & 3 & 1 & 1 \\
\hline
\end{tabular}




\section{Continued}

\begin{tabular}{|c|c|c|c|c|c|c|c|c|c|}
\hline & VAR(2) & 5 & 5 & 5 & 3 & 5 & 5 & 5 & 5 \\
\hline & BVAR1 & 2 & 2 & 2 & 2 & 4 & 3 & 4 & 4 \\
\hline \multirow[t]{5}{*}{-0.9} & BVAR2 & 1 & 1 & 1 & 1 & 1 & 1 & 2 & 2 \\
\hline & BVAR3 & 4 & 4 & 4 & 5 & 3 & 4 & 3 & 3 \\
\hline & BVAR4 & 3 & 3 & 3 & 4 & 2 & 2 & 1 & 1 \\
\hline & VAR(2) & 3 & 1 & 4 & 2 & 5 & 5 & 5 & 5 \\
\hline & BVAR1 & 2 & 3 & 2 & 3 & 3 & 3 & 4 & 4 \\
\hline \multirow[t]{5}{*}{-0.85} & BVAR2 & 1 & 2 & 1 & 1 & 1 & 1 & 2 & 2 \\
\hline & BVAR3 & 5 & 5 & 5 & 5 & 4 & 4 & 3 & 3 \\
\hline & BVAR4 & 4 & 4 & 3 & 4 & 2 & 2 & 1 & 1 \\
\hline & VAR(2) & 2 & 1 & 3 & 1 & 4 & 1 & 5 & 5 \\
\hline & BVAR1 & 3 & 3 & 2 & 3 & 3 & 3 & 4 & 4 \\
\hline \multirow[t]{5}{*}{-0.8} & BVAR2 & 1 & 2 & 1 & 2 & 1 & 2 & 2 & 2 \\
\hline & BVAR3 & 5 & 5 & 5 & 5 & 5 & 5 & 3 & 3 \\
\hline & BVAR4 & 4 & 4 & 4 & 4 & 2 & 4 & 1 & 1 \\
\hline & VAR(2) & 1 & 1 & 1 & 1 & 1 & 1 & 5 & 2 \\
\hline & BVAR1 & 3 & 3 & 4 & 3 & 4 & 3 & 4 & 5 \\
\hline \multirow[t]{5}{*}{0.8} & BVAR2 & 4 & 4 & 3 & 4 & 3 & 4 & 3 & 4 \\
\hline & BVAR3 & 5 & 5 & 5 & 5 & 5 & 5 & 2 & 3 \\
\hline & BVAR4 & 2 & 2 & 2 & 2 & 2 & 2 & 1 & 1 \\
\hline & VAR(2) & 5 & 1 & 5 & 5 & 5 & 5 & 5 & 5 \\
\hline & BVAR1 & 3 & 3 & 3 & 3 & 2 & 2 & 4 & 4 \\
\hline \multirow[t]{5}{*}{0.85} & BVAR2 & 2 & 4 & 2 & 2 & 4 & 4 & 3 & 3 \\
\hline & BVAR3 & 4 & 5 & 4 & 4 & 3 & 3 & 2 & 2 \\
\hline & BVAR4 & 1 & 2 & 1 & 1 & 1 & 1 & 1 & 1 \\
\hline & VAR(2) & 5 & 5 & 5 & 5 & 5 & 5 & 5 & 5 \\
\hline & BVAR1 & 4 & 3 & 4 & 4 & 4 & 4 & 4 & 4 \\
\hline \multirow[t]{5}{*}{0.9} & BVAR2 & 2 & 2 & 3 & 3 & 3 & 3 & 3 & 3 \\
\hline & BVAR3 & 3 & 4 & 2 & 2 & 2 & 2 & 2 & 2 \\
\hline & BVAR4 & 1 & 1 & 1 & 1 & 1 & 1 & 1 & 1 \\
\hline & VAR(2) & 5 & 5 & 5 & 5 & 5 & 5 & 5 & 5 \\
\hline & BVAR1 & 4 & 4 & 4 & 3 & 4 & 3 & 3 & 3 \\
\hline \multirow[t]{5}{*}{0.95} & BVAR2 & 3 & 3 & 3 & 4 & 3 & 4 & 4 & 4 \\
\hline & BVAR3 & 2 & 2 & 2 & 2 & 2 & 2 & 2 & 2 \\
\hline & BVAR4 & 1 & 1 & 1 & 1 & 1 & 1 & 1 & 1 \\
\hline & VAR(2) & 5 & 5 & 5 & 5 & 5 & 5 & 5 & 5 \\
\hline & BVAR1 & 4 & 2 & 4 & 3 & 3 & 3 & 4 & 3 \\
\hline \multirow[t]{3}{*}{0.99} & BVAR2 & 3 & 3 & 3 & 4 & 4 & 4 & 3 & 4 \\
\hline & BVAR3 & 2 & 4 & 1 & 1 & 2 & 2 & 2 & 2 \\
\hline & BVAR4 & 1 & 1 & 2 & 2 & 1 & 1 & 1 & 1 \\
\hline
\end{tabular}


M. O. Adenomon et al.

Table B4. The ranks of the performances of the model for $T=64$.

\begin{tabular}{|c|c|c|c|c|c|c|c|c|c|c|c|c|c|}
\hline \multirow{3}{*}{$\begin{array}{c}T=64 \\
\text { AUTOCO } \\
\text { LEVELS }(\delta)\end{array}$} & \multirow{3}{*}{ Models } & \multicolumn{12}{|c|}{ COLLINERITY $(\rho)$} \\
\hline & & \multicolumn{2}{|c|}{-0.99} & \multicolumn{2}{|c|}{-0.95} & \multicolumn{2}{|c|}{-0.9} & \multicolumn{2}{|c|}{-0.85} & \multicolumn{2}{|c|}{-0.8} & \multicolumn{2}{|c|}{0.8} \\
\hline & & RMSE & MAE & RMSE & MAE & RMSE & MAE & RMSE & MAE & RMSE & MAE & RMSE & MAE \\
\hline \multirow{5}{*}{-0.99} & VAR(2) & 5 & 5 & 5 & 5 & 5 & 5 & 5 & 5 & 5 & 5 & 5 & 5 \\
\hline & BVAR1 & 4 & 4 & 4 & 4 & 4 & 4 & 4 & 4 & 4 & 4 & 4 & 4 \\
\hline & BVAR2 & 3 & 3 & 3 & 3 & 3 & 3 & 3 & 3 & 3 & 3 & 3 & 3 \\
\hline & BVAR3 & 2 & 2 & 2 & 2 & 2 & 2 & 2 & 2 & 2 & 2 & 2 & 2 \\
\hline & BVAR4 & 1 & 1 & 1 & 1 & 1 & 1 & 1 & 1 & 1 & 1 & 1 & 1 \\
\hline \multirow{5}{*}{-0.95} & VAR(2) & 5 & 5 & 5 & 5 & 5 & 5 & 5 & 5 & 5 & 5 & 5 & 5 \\
\hline & BVAR1 & 2 & 2 & 2 & 2 & 2 & 3 & 3 & 3 & 4 & 1 & 3 & 2 \\
\hline & BVAR2 & 1 & 1 & 1 & 1 & 4 & 2 & 2 & 1 & 3 & 3 & 1 & 1 \\
\hline & BVAR3 & 3 & 3 & 3 & 3 & 3 & 4 & 4 & 4 & 2 & 4 & 4 & 4 \\
\hline & BVAR4 & 4 & 4 & 4 & 4 & 1 & 1 & 1 & 2 & 1 & 2 & 2 & 3 \\
\hline \multirow{5}{*}{-0.9} & VAR(2) & 5 & 5 & 5 & 5 & 5 & 5 & 5 & 5 & 5 & 5 & 4 & 1 \\
\hline & BVAR1 & 4 & 3 & 3 & 3 & 4 & 3 & 2 & 1 & 4 & 4 & 2 & 3 \\
\hline & BVAR2 & 1 & 2 & 1 & 1 & 2 & 1 & 4 & 4 & 3 & 3 & 1 & 2 \\
\hline & BVAR3 & 2 & 1 & 2 & 2 & 3 & 4 & 3 & 3 & 2 & 2 & 5 & 5 \\
\hline & BVAR4 & 3 & 4 & 4 & 4 & 1 & 2 & 1 & 2 & 1 & 1 & 3 & 4 \\
\hline \multirow{5}{*}{-0.85} & VAR(2) & 5 & 5 & 5 & 5 & 5 & 5 & 5 & 5 & 5 & 5 & 1 & 1 \\
\hline & BVAR1 & 4 & 3 & 1 & 1 & 3 & 3 & 4 & 4 & 4 & 4 & 3 & 3 \\
\hline & BVAR2 & 1 & 1 & 4 & 4 & 4 & 4 & 3 & 3 & 3 & 3 & 2 & 2 \\
\hline & BVAR3 & 3 & 4 & 3 & 3 & 2 & 2 & 2 & 2 & 2 & 2 & 5 & 5 \\
\hline & BVAR4 & 2 & 2 & 2 & 2 & 1 & 1 & 1 & 1 & 1 & 1 & 4 & 4 \\
\hline \multirow{5}{*}{-0.8} & VAR(2) & 5 & 5 & 5 & 5 & 5 & 5 & 5 & 5 & 4 & 5 & 1 & 1 \\
\hline & BVAR1 & 4 & 3 & 3 & 3 & 3 & 3 & 4 & 4 & 5 & 4 & 3 & 3 \\
\hline & BVAR2 & 1 & 1 & 4 & 4 & 4 & 4 & 3 & 3 & 3 & 3 & 2 & 2 \\
\hline & BVAR3 & 3 & 4 & 2 & 2 & 2 & 2 & 2 & 2 & 2 & 2 & 5 & 5 \\
\hline & BVAR4 & 2 & 2 & 1 & 1 & 1 & 1 & 1 & 1 & 1 & 1 & 4 & 4 \\
\hline \multirow{5}{*}{0.8} & VAR(2) & 3 & 3 & 3 & 3 & 3 & 3 & 3 & 3 & 2 & 2 & 1 & 1 \\
\hline & BVAR1 & 5 & 5 & 5 & 5 & 5 & 5 & 5 & 5 & 5 & 5 & 5 & 5 \\
\hline & BVAR2 & 4 & 4 & 4 & 4 & 4 & 4 & 4 & 4 & 4 & 4 & 4 & 3 \\
\hline & BVAR3 & 2 & 2 & 2 & 2 & 2 & 2 & 2 & 2 & 3 & 3 & 3 & 4 \\
\hline & BVAR4 & 1 & 1 & 1 & 1 & 1 & 1 & 1 & 1 & 1 & 1 & 2 & 2 \\
\hline \multirow{5}{*}{0.85} & VAR(2) & 4 & 4 & 5 & 5 & 5 & 4 & 3 & 3 & 3 & 3 & 1 & 1 \\
\hline & BVAR1 & 5 & 5 & 4 & 4 & 4 & 5 & 5 & 5 & 5 & 5 & 5 & 5 \\
\hline & BVAR2 & 3 & 3 & 3 & 3 & 3 & 3 & 4 & 4 & 4 & 4 & 4 & 4 \\
\hline & BVAR3 & 2 & 2 & 2 & 2 & 2 & 2 & 2 & 2 & 2 & 2 & 3 & 3 \\
\hline & BVAR4 & 1 & 1 & 1 & 1 & 1 & 1 & 1 & 1 & 1 & 1 & 2 & 2 \\
\hline \multirow{5}{*}{0.9} & VAR(2) & 5 & 5 & 5 & 5 & 5 & 5 & 5 & 5 & 5 & 5 & 2 & 1 \\
\hline & BVAR1 & 4 & 4 & 4 & 4 & 4 & 4 & 4 & 4 & 4 & 4 & 5 & 5 \\
\hline & BVAR2 & 3 & 3 & 3 & 3 & 3 & 3 & 3 & 3 & 3 & 3 & 4 & 4 \\
\hline & BVAR3 & 2 & 2 & 2 & 2 & 2 & 2 & 2 & 2 & 2 & 2 & 3 & 3 \\
\hline & BVAR4 & 1 & 1 & 1 & 1 & 1 & 1 & 1 & 1 & 1 & 1 & 1 & 2 \\
\hline
\end{tabular}




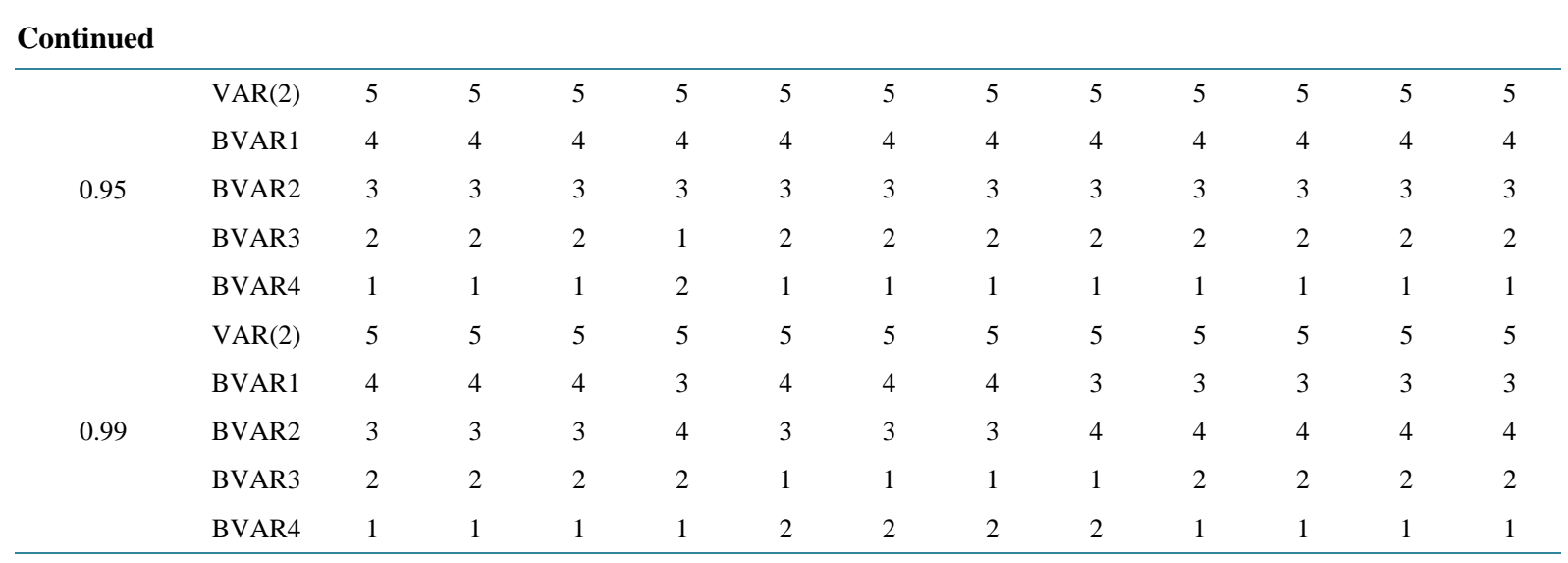

\begin{tabular}{|c|c|c|c|c|c|c|c|c|c|}
\hline \multirow{3}{*}{$\begin{array}{c}T=64 \\
\text { AUTOCO } \\
\text { LEVELS }(\delta)\end{array}$} & \multirow{3}{*}{ Models } & \multicolumn{8}{|c|}{ COLLINEARITY $(\rho)$} \\
\hline & & \multicolumn{2}{|c|}{0.85} & \multicolumn{2}{|c|}{0.9} & \multicolumn{2}{|c|}{0.95} & \multicolumn{2}{|c|}{0.99} \\
\hline & & RMSE & MAE & RMSE & MAE & RMSE & MAE & RMSE & MAE \\
\hline \multirow{5}{*}{-0.99} & VAR(2) & 5 & 5 & 5 & 5 & 5 & 5 & 5 & 5 \\
\hline & BVAR1 & 4 & 4 & 4 & 4 & 4 & 4 & 4 & 4 \\
\hline & BVAR2 & 3 & 3 & 3 & 2 & 3 & 3 & 3 & 3 \\
\hline & BVAR3 & 2 & 2 & 2 & 3 & 2 & 2 & 2 & 2 \\
\hline & BVAR4 & 1 & 1 & 1 & 1 & 1 & 1 & 1 & 1 \\
\hline \multirow{5}{*}{-0.95} & VAR(2) & 5 & 5 & 5 & 5 & 5 & 5 & 5 & 5 \\
\hline & BVAR1 & 3 & 2 & 2 & 2 & 2 & 2 & 2 & 2 \\
\hline & BVAR2 & 1 & 1 & 1 & 1 & 1 & 1 & 1 & 1 \\
\hline & BVAR3 & 4 & 4 & 4 & 4 & 4 & 4 & 4 & 4 \\
\hline & BVAR4 & 2 & 3 & 3 & 3 & 3 & 3 & 3 & 3 \\
\hline \multirow{5}{*}{-0.9} & VAR(2) & 5 & 1 & 5 & 3 & 5 & 5 & 5 & 5 \\
\hline & BVAR1 & 2 & 3 & 2 & 2 & 2 & 2 & 3 & 3 \\
\hline & BVAR2 & 1 & 2 & 1 & 1 & 1 & 1 & 1 & 1 \\
\hline & BVAR3 & 4 & 5 & 4 & 5 & 4 & 4 & 4 & 4 \\
\hline & BVAR4 & 3 & 4 & 3 & 4 & 3 & 3 & 2 & 2 \\
\hline \multirow{5}{*}{-0.85} & VAR(2) & 1 & 1 & 3 & 1 & 4 & 3 & 5 & 5 \\
\hline & BVAR1 & 3 & 3 & 2 & 3 & 2 & 2 & 3 & 3 \\
\hline & BVAR2 & 2 & 2 & 1 & 2 & 1 & 1 & 1 & 1 \\
\hline & BVAR3 & 5 & 5 & 5 & 5 & 5 & 5 & 4 & 4 \\
\hline & BVAR4 & 4 & 4 & 4 & 4 & 3 & 4 & 2 & 2 \\
\hline \multirow{5}{*}{-0.8} & VAR(2) & 1 & 1 & 1 & 1 & 1 & 1 & 5 & 5 \\
\hline & BVAR1 & 3 & 3 & 3 & 3 & 3 & 3 & 3 & 3 \\
\hline & BVAR2 & 2 & 2 & 2 & 2 & 2 & 2 & 1 & 1 \\
\hline & BVAR3 & 5 & 5 & 5 & 5 & 5 & 5 & 4 & 4 \\
\hline & BVAR4 & 4 & 4 & 4 & 4 & 4 & 4 & 2 & 2 \\
\hline \multirow{5}{*}{0.8} & VAR(2) & 1 & 1 & 1 & 1 & 1 & 1 & 1 & 1 \\
\hline & BVAR1 & 5 & 5 & 5 & 5 & 5 & 5 & 5 & 5 \\
\hline & BVAR2 & 4 & 4 & 4 & 4 & 4 & 4 & 4 & 4 \\
\hline & BVAR3 & 3 & 3 & 3 & 3 & 3 & 3 & 3 & 3 \\
\hline & BVAR4 & 2 & 2 & 2 & 2 & 2 & 2 & 2 & 2 \\
\hline
\end{tabular}




\section{Continued}

\begin{tabular}{|c|c|c|c|c|c|c|c|c|c|}
\hline & VAR(2) & 1 & 1 & 1 & 1 & 1 & 1 & 1 & 1 \\
\hline & BVAR1 & 5 & 5 & 5 & 5 & 5 & 5 & 5 & 5 \\
\hline \multirow[t]{5}{*}{0.85} & BVAR2 & 4 & 4 & 4 & 4 & 4 & 4 & 4 & 4 \\
\hline & BVAR3 & 3 & 3 & 3 & 3 & 3 & 3 & 3 & 3 \\
\hline & BVAR4 & 2 & 2 & 2 & 2 & 2 & 2 & 2 & 2 \\
\hline & VAR(2) & 4 & 3 & 3 & 2 & 5 & 5 & 5 & 5 \\
\hline & BVAR1 & 5 & 5 & 5 & 5 & 4 & 4 & 4 & 4 \\
\hline \multirow[t]{5}{*}{0.9} & BVAR2 & 3 & 4 & 4 & 4 & 3 & 3 & 3 & 3 \\
\hline & BVAR3 & 2 & 2 & 2 & 3 & 2 & 2 & 2 & 2 \\
\hline & BVAR4 & 1 & 1 & 1 & 1 & 1 & 1 & 1 & 1 \\
\hline & VAR(2) & 5 & 5 & 5 & 5 & 5 & 5 & 5 & 5 \\
\hline & BVAR1 & 4 & 4 & 4 & 4 & 4 & 4 & 4 & 4 \\
\hline \multirow[t]{5}{*}{0.95} & BVAR2 & 3 & 3 & 3 & 3 & 3 & 3 & 3 & 3 \\
\hline & BVAR3 & 2 & 2 & 2 & 2 & 2 & 2 & 2 & 2 \\
\hline & BVAR4 & 1 & 1 & 1 & 1 & 1 & 1 & 1 & 1 \\
\hline & VAR(2) & 5 & 5 & 5 & 5 & 5 & 5 & 5 & 5 \\
\hline & BVAR1 & 3 & 3 & 4 & 3 & 4 & 4 & 3 & 3 \\
\hline \multirow[t]{3}{*}{0.99} & BVAR2 & 4 & 4 & 3 & 4 & 3 & 3 & 4 & 4 \\
\hline & BVAR3 & 2 & 2 & 2 & 2 & 2 & 2 & 2 & 2 \\
\hline & BVAR4 & 1 & 1 & 1 & 1 & 1 & 1 & 1 & 1 \\
\hline
\end{tabular}

Table B5. The ranks of the performances of the model for $T=128$.

\begin{tabular}{|c|c|c|c|c|c|c|c|c|c|c|c|c|c|}
\hline \multirow{3}{*}{$\begin{array}{c}T=128 \\
\text { AUTOCO } \\
\text { LEVELS }(\delta)\end{array}$} & \multirow{3}{*}{ Models } & \multicolumn{12}{|c|}{ COLLINERITY $(\rho)$} \\
\hline & & \multicolumn{2}{|c|}{-0.99} & \multicolumn{2}{|c|}{-0.95} & \multicolumn{2}{|c|}{-0.9} & \multicolumn{2}{|c|}{-0.85} & \multicolumn{2}{|c|}{-0.8} & \multicolumn{2}{|c|}{0.8} \\
\hline & & RMSE & MAE & RMSE & MAE & RMSE & MAE & RMSE & MAE & RMSE & MAE & RMSE & MAE \\
\hline \multirow{5}{*}{-0.99} & $\operatorname{VAR}(2)$ & 5 & 5 & 5 & 5 & 5 & 5 & 5 & 5 & 5 & 5 & 5 & 5 \\
\hline & BVAR1 & 4 & 4 & 4 & 4 & 3 & 3 & 3 & 3 & 3 & 3 & 4 & 4 \\
\hline & BVAR2 & 3 & 3 & 3 & 3 & 2 & 2 & 1 & 1 & 1 & 1 & 3 & 3 \\
\hline & BVAR3 & 2 & 2 & 1 & 1 & 1 & 1 & 2 & 2 & 2 & 2 & 2 & 2 \\
\hline & BVAR4 & 1 & 1 & 2 & 2 & 4 & 4 & 4 & 4 & 4 & 4 & 1 & 1 \\
\hline \multirow{5}{*}{-0.95} & $\operatorname{VAR}(2)$ & 5 & 5 & 5 & 5 & 5 & 5 & 5 & 5 & 5 & 5 & 5 & 4 \\
\hline & BVAR1 & 1 & 2 & 1 & 2 & 1 & 1 & 1 & 1 & 1 & 1 & 4 & 5 \\
\hline & BVAR2 & 2 & 1 & 2 & 1 & 2 & 2 & 2 & 2 & 3 & 2 & 2 & 2 \\
\hline & BVAR3 & 3 & 3 & 3 & 3 & 3 & 3 & 3 & 3 & 2 & 3 & 3 & 3 \\
\hline & BVAR4 & 4 & 4 & 4 & 4 & 4 & 4 & 4 & 4 & 4 & 4 & 1 & 1 \\
\hline \multirow{5}{*}{-0.9} & VAR(2) & 5 & 5 & 5 & 5 & 5 & 5 & 5 & 5 & 5 & 5 & 1 & 1 \\
\hline & BVAR1 & 2 & 2 & 1 & 1 & 4 & 2 & 4 & 4 & 4 & 4 & 5 & 5 \\
\hline & BVAR2 & 1 & 1 & 2 & 2 & 1 & 1 & 3 & 3 & 3 & 3 & 3 & 3 \\
\hline & BVAR3 & 3 & 3 & 4 & 3 & 3 & 3 & 2 & 2 & 2 & 2 & 4 & 4 \\
\hline & BVAR4 & 4 & 4 & 3 & 4 & 2 & 4 & 1 & 1 & 1 & 1 & 2 & 2 \\
\hline \multirow{5}{*}{-0.85} & VAR(2) & 5 & 5 & 5 & 5 & 5 & 5 & 4 & 5 & 3 & 4 & 1 & 1 \\
\hline & BVAR1 & 1 & 1 & 2 & 2 & 4 & 4 & 5 & 4 & 5 & 5 & 5 & 5 \\
\hline & BVAR2 & 2 & 2 & 4 & 3 & 3 & 3 & 3 & 3 & 4 & 3 & 3 & 3 \\
\hline & BVAR3 & 3 & 3 & 3 & 4 & 2 & 2 & 2 & 2 & 2 & 2 & 4 & 4 \\
\hline & BVAR4 & 4 & 4 & 1 & 1 & 1 & 1 & 1 & 1 & 1 & 1 & 2 & 2 \\
\hline
\end{tabular}




\begin{tabular}{|c|c|c|c|c|c|c|c|c|c|c|c|c|c|}
\hline \multicolumn{14}{|c|}{ Continued } \\
\hline & VAR(2) & 5 & 5 & 5 & 5 & 2 & 2 & 2 & 2 & 1 & 2 & 1 & 1 \\
\hline & BVAR1 & 2 & 1 & 3 & 3 & 5 & 5 & 5 & 5 & 5 & 5 & 5 & 5 \\
\hline \multirow[t]{5}{*}{-0.8} & BVAR2 & 1 & 2 & 4 & 4 & 4 & 4 & 4 & 4 & 4 & 4 & 3 & 3 \\
\hline & BVAR3 & 4 & 4 & 2 & 2 & 3 & 3 & 3 & 3 & 3 & 3 & 4 & 4 \\
\hline & BVAR4 & 3 & 3 & 1 & 1 & 1 & 1 & 1 & 1 & 2 & 1 & 2 & 2 \\
\hline & VAR(2) & 2 & 2 & 3 & 3 & 2 & 2 & 1 & 1 & 1 & 1 & 1 & 1 \\
\hline & BVAR1 & 5 & 5 & 5 & 5 & 5 & 5 & 5 & 5 & 5 & 5 & 5 & 5 \\
\hline \multirow[t]{5}{*}{0.8} & BVAR2 & 4 & 4 & 4 & 4 & 4 & 4 & 4 & 4 & 4 & 4 & 3 & 3 \\
\hline & BVAR3 & 3 & 3 & 2 & 2 & 3 & 3 & 3 & 3 & 3 & 3 & 4 & 4 \\
\hline & BVAR4 & 1 & 1 & 1 & 1 & 1 & 1 & 2 & 2 & 2 & 2 & 2 & 2 \\
\hline & VAR(2) & 3 & 3 & 3 & 4 & 3 & 3 & 2 & 2 & 1 & 2 & 1 & 1 \\
\hline & BVAR1 & 5 & 5 & 5 & 5 & 5 & 5 & 5 & 5 & 5 & 5 & 5 & 5 \\
\hline \multirow[t]{5}{*}{0.85} & BVAR2 & 4 & 4 & 4 & 3 & 4 & 4 & 4 & 4 & 4 & 4 & 3 & 3 \\
\hline & BVAR3 & 2 & 2 & 2 & 2 & 2 & 2 & 3 & 3 & 3 & 3 & 4 & 4 \\
\hline & BVAR4 & 1 & 1 & 1 & 1 & 1 & 1 & 1 & 1 & 2 & 1 & 2 & 2 \\
\hline & VAR(2) & 5 & 5 & 5 & 5 & 5 & 5 & 4 & 5 & 4 & 4 & 1 & 1 \\
\hline & BVAR1 & 4 & 4 & 4 & 4 & 4 & 4 & 5 & 4 & 5 & 5 & 5 & 5 \\
\hline \multirow[t]{5}{*}{0.9} & BVAR2 & 3 & 3 & 3 & 3 & 3 & 3 & 3 & 3 & 3 & 3 & 4 & 3 \\
\hline & BVAR3 & 2 & 2 & 2 & 2 & 2 & 2 & 2 & 2 & 2 & 2 & 3 & 4 \\
\hline & BVAR4 & 1 & 1 & 1 & 1 & 1 & 1 & 1 & 1 & 1 & 1 & 2 & 2 \\
\hline & VAR(2) & 5 & 5 & 5 & 5 & 5 & 5 & 5 & 5 & 5 & 5 & 5 & 4 \\
\hline & BVAR1 & 4 & 4 & 4 & 4 & 4 & 4 & 4 & 4 & 4 & 4 & 4 & 5 \\
\hline \multirow[t]{5}{*}{0.95} & BVAR2 & 3 & 3 & 3 & 3 & 3 & 3 & 3 & 3 & 3 & 3 & 3 & 3 \\
\hline & BVAR3 & 2 & 2 & 2 & 2 & 2 & 2 & 2 & 2 & 2 & 2 & 2 & 2 \\
\hline & BVAR4 & 1 & 1 & 1 & 1 & 1 & 1 & 1 & 1 & 1 & 1 & 1 & 1 \\
\hline & VAR(2) & 5 & 5 & 5 & 5 & 5 & 5 & 5 & 5 & 5 & 5 & 5 & 5 \\
\hline & BVAR1 & 4 & 4 & 3 & 3 & 3 & 3 & 3 & 2 & 3 & 3 & 4 & 4 \\
\hline \multirow[t]{3}{*}{0.99} & BVAR2 & 3 & 3 & 4 & 4 & 4 & 4 & 2 & 3 & 4 & 4 & 3 & 3 \\
\hline & BVAR3 & 1 & 1 & 2 & 2 & 1 & 1 & 1 & 1 & 1 & 1 & 2 & 1 \\
\hline & BVAR4 & 2 & 2 & 1 & 1 & 2 & 2 & 4 & 4 & 2 & 2 & 1 & 2 \\
\hline
\end{tabular}

\begin{tabular}{|c|c|c|c|c|c|c|c|c|c|}
\hline \multirow{3}{*}{$\begin{array}{c}T=128 \\
\text { AUTOCO } \\
\text { LEVELS }(\delta)\end{array}$} & \multirow{3}{*}{ Models } & \multicolumn{8}{|c|}{ COLLINEARITY $(\rho)$} \\
\hline & & \multicolumn{2}{|c|}{0.85} & \multicolumn{2}{|c|}{0.9} & \multicolumn{2}{|c|}{0.95} & \multicolumn{2}{|c|}{0.99} \\
\hline & & RMSE & MAE & RMSE & MAE & RMSE & MAE & RMSE & MAE \\
\hline \multirow{5}{*}{-0.99} & VAR(2) & 5 & 5 & 5 & 5 & 5 & 5 & 5 & 5 \\
\hline & BVAR1 & 4 & 4 & 4 & 4 & 3 & 3 & 4 & 4 \\
\hline & BVAR2 & 3 & 3 & 3 & 3 & 4 & 4 & 3 & 3 \\
\hline & BVAR3 & 2 & 2 & 2 & 2 & 2 & 2 & 1 & 2 \\
\hline & BVAR4 & 1 & 1 & 1 & 1 & 1 & 1 & 2 & 1 \\
\hline \multirow{5}{*}{-0.95} & VAR(2) & 5 & 3 & 5 & 4 & 5 & 5 & 5 & 5 \\
\hline & BVAR1 & 4 & 5 & 4 & 5 & 4 & 4 & 2 & 2 \\
\hline & BVAR2 & 2 & 2 & 2 & 2 & 1 & 1 & 1 & 1 \\
\hline & BVAR3 & 3 & 4 & 3 & 3 & 3 & 3 & 3 & 3 \\
\hline & BVAR4 & 1 & 1 & 1 & 1 & 2 & 2 & 4 & 4 \\
\hline
\end{tabular}


M. O. Adenomon et al.

\section{Continued}

\begin{tabular}{|c|c|c|c|c|c|c|c|c|c|}
\hline & VAR(2) & 1 & 1 & 3 & 1 & 3 & 2 & 5 & 5 \\
\hline & BVAR1 & 5 & 5 & 5 & 5 & 5 & 5 & 3 & 3 \\
\hline \multirow[t]{5}{*}{-0.9} & BVAR2 & 3 & 3 & 2 & 3 & 2 & 3 & 1 & 1 \\
\hline & BVAR3 & 4 & 4 & 4 & 4 & 4 & 4 & 4 & 4 \\
\hline & BVAR4 & 2 & 2 & 1 & 2 & 1 & 1 & 2 & 2 \\
\hline & VAR(2) & 1 & 1 & 1 & 1 & 1 & 1 & 5 & 5 \\
\hline & BVAR1 & 5 & 5 & 5 & 5 & 5 & 5 & 3 & 3 \\
\hline \multirow[t]{5}{*}{-0.85} & BVAR2 & 3 & 3 & 3 & 3 & 3 & 3 & 1 & 1 \\
\hline & BVAR3 & 4 & 4 & 4 & 4 & 4 & 4 & 4 & 4 \\
\hline & BVAR4 & 2 & 2 & 2 & 2 & 2 & 2 & 2 & 2 \\
\hline & VAR(2) & 1 & 1 & 1 & 1 & 1 & 1 & 3 & 2 \\
\hline & BVAR1 & 5 & 5 & 5 & 5 & 4 & 4 & 4 & 4 \\
\hline \multirow[t]{5}{*}{-0.8} & BVAR2 & 3 & 3 & 3 & 3 & 3 & 3 & 1 & 1 \\
\hline & BVAR3 & 4 & 4 & 4 & 4 & 5 & 5 & 5 & 5 \\
\hline & BVAR4 & 2 & 2 & 2 & 2 & 2 & 2 & 2 & 3 \\
\hline & VAR(2) & 1 & 1 & 1 & 1 & 1 & 1 & 1 & 1 \\
\hline & BVAR1 & 5 & 5 & 5 & 5 & 5 & 5 & 5 & 5 \\
\hline \multirow[t]{5}{*}{0.8} & BVAR2 & 3 & 3 & 3 & 3 & 4 & 4 & 4 & 4 \\
\hline & BVAR3 & 4 & 4 & 4 & 4 & 3 & 3 & 3 & 3 \\
\hline & BVAR4 & 2 & 2 & 2 & 2 & 2 & 2 & 2 & 2 \\
\hline & VAR(2) & 1 & 1 & 1 & 1 & 1 & 1 & 1 & 1 \\
\hline & BVAR1 & 5 & 5 & 5 & 5 & 5 & 5 & 5 & 5 \\
\hline \multirow[t]{5}{*}{0.85} & BVAR2 & 3 & 3 & 4 & 4 & 4 & 4 & 4 & 4 \\
\hline & BVAR3 & 4 & 4 & 3 & 3 & 3 & 3 & 3 & 3 \\
\hline & BVAR4 & 2 & 2 & 2 & 2 & 2 & 2 & 2 & 2 \\
\hline & VAR(2) & 1 & 1 & 1 & 1 & 2 & 2 & 3 & 3 \\
\hline & BVAR1 & 5 & 5 & 5 & 5 & 5 & 5 & 5 & 5 \\
\hline \multirow[t]{5}{*}{0.9} & BVAR2 & 4 & 4 & 4 & 4 & 4 & 4 & 4 & 4 \\
\hline & BVAR3 & 3 & 3 & 3 & 3 & 3 & 3 & 2 & 2 \\
\hline & BVAR4 & 2 & 2 & 2 & 2 & 1 & 1 & 1 & 1 \\
\hline & VAR(2) & 5 & 5 & 5 & 5 & 5 & 5 & 5 & 5 \\
\hline & BVAR1 & 4 & 4 & 4 & 4 & 4 & 4 & 4 & 4 \\
\hline \multirow[t]{5}{*}{0.95} & BVAR2 & 3 & 3 & 3 & 3 & 3 & 3 & 3 & 3 \\
\hline & BVAR3 & 2 & 2 & 2 & 2 & 2 & 2 & 2 & 2 \\
\hline & BVAR4 & 1 & 1 & 1 & 1 & 1 & 1 & 1 & 1 \\
\hline & VAR(2) & 5 & 5 & 5 & 5 & 5 & 5 & 5 & 5 \\
\hline & BVAR1 & 3 & 3 & 4 & 4 & 3 & 3 & 4 & 4 \\
\hline \multirow[t]{3}{*}{0.99} & BVAR2 & 4 & 4 & 2 & 2 & 4 & 4 & 3 & 3 \\
\hline & BVAR3 & 2 & 2 & 3 & 3 & 2 & 2 & 2 & 2 \\
\hline & BVAR4 & 1 & 1 & 1 & 1 & 1 & 1 & 1 & 1 \\
\hline
\end{tabular}


Table B6. The ranks of the performances of the model for $T=256$.

\begin{tabular}{|c|c|c|c|c|c|c|c|c|c|c|c|c|c|}
\hline \multirow{3}{*}{$\begin{array}{c}T=256 \\
\text { AUTOCO } \\
\text { LEVELS }(\delta)\end{array}$} & \multirow{3}{*}{ Models } & \multicolumn{12}{|c|}{ COLLINERITY $(\rho)$} \\
\hline & & \multicolumn{2}{|c|}{-0.99} & \multicolumn{2}{|c|}{-0.95} & \multicolumn{2}{|c|}{-0.9} & \multicolumn{2}{|c|}{-0.85} & \multicolumn{2}{|c|}{-0.8} & \multicolumn{2}{|c|}{0.8} \\
\hline & & RMSE & MAE & RMSE & MAE & RMSE & MAE & RMSE & MAE & RMSE & MAE & RMSE & MAE \\
\hline \multirow{5}{*}{-0.99} & VAR(2) & 5 & 5 & 5 & 5 & 5 & 5 & 5 & 5 & 5 & 5 & 5 & 5 \\
\hline & BVAR1 & 2 & 2 & 1 & 1 & 2 & 2 & 1 & 1 & 1 & 1 & 4 & 4 \\
\hline & BVAR2 & 1 & 1 & 2 & 2 & 1 & 1 & 2 & 2 & 2 & 2 & 3 & 3 \\
\hline & BVAR3 & 3 & 3 & 3 & 3 & 3 & 3 & 3 & 3 & 3 & 3 & 2 & 2 \\
\hline & BVAR4 & 4 & 4 & 4 & 4 & 4 & 4 & 4 & 4 & 4 & 4 & 1 & 1 \\
\hline \multirow{5}{*}{-0.95} & VAR(2) & 5 & 5 & 5 & 5 & 5 & 5 & 5 & 5 & 5 & 5 & 4 & 2 \\
\hline & BVAR1 & 1 & 1 & 1 & 1 & 1 & 1 & 1 & 1 & 1 & 1 & 5 & 5 \\
\hline & BVAR2 & 2 & 2 & 2 & 2 & 2 & 2 & 2 & 2 & 2 & 2 & 2 & 3 \\
\hline & BVAR3 & 3 & 3 & 3 & 3 & 3 & 3 & 3 & 3 & 3 & 3 & 3 & 4 \\
\hline & BVAR4 & 4 & 4 & 4 & 4 & 4 & 4 & 4 & 4 & 4 & 4 & 1 & 1 \\
\hline \multirow{5}{*}{-0.9} & VAR(2) & 5 & 5 & 5 & 5 & 5 & 5 & 5 & 5 & 4 & 5 & 1 & 1 \\
\hline & BVAR1 & 1 & 1 & 1 & 1 & 3 & 1 & 4 & 4 & 5 & 4 & 5 & 5 \\
\hline & BVAR2 & 2 & 2 & 2 & 2 & 2 & 2 & 1 & 1 & 3 & 3 & 4 & 4 \\
\hline & BVAR3 & 3 & 3 & 3 & 3 & 1 & 3 & 2 & 2 & 2 & 2 & 3 & 3 \\
\hline & BVAR4 & 4 & 4 & 4 & 4 & 4 & 4 & 3 & 3 & 1 & 1 & 2 & 2 \\
\hline \multirow{5}{*}{-0.85} & VAR(2) & 5 & 5 & 5 & 5 & 3 & 4 & 3 & 4 & 2 & 2 & 1 & 1 \\
\hline & BVAR1 & 2 & 2 & 4 & 4 & 5 & 5 & 5 & 5 & 5 & 5 & 5 & 5 \\
\hline & BVAR2 & 1 & 1 & 2 & 2 & 4 & 3 & 4 & 3 & 4 & 4 & 4 & 4 \\
\hline & BVAR3 & 4 & 4 & 3 & 3 & 2 & 2 & 2 & 2 & 3 & 3 & 3 & 3 \\
\hline & BVAR4 & 3 & 3 & 1 & 1 & 1 & 1 & 1 & 1 & 1 & 1 & 2 & 2 \\
\hline \multirow{5}{*}{-0.8} & VAR(2) & 5 & 5 & 3 & 4 & 2 & 2 & 2 & 2 & 1 & 1 & 1 & 1 \\
\hline & BVAR1 & 1 & 1 & 5 & 5 & 5 & 5 & 5 & 5 & 5 & 5 & 5 & 5 \\
\hline & BVAR2 & 2 & 3 & 4 & 3 & 4 & 4 & 4 & 4 & 4 & 4 & 4 & 4 \\
\hline & BVAR3 & 4 & 4 & 2 & 2 & 3 & 3 & 3 & 3 & 3 & 3 & 3 & 3 \\
\hline & BVAR4 & 3 & 2 & 1 & 1 & 1 & 1 & 1 & 1 & 2 & 2 & 2 & 2 \\
\hline \multirow{5}{*}{0.8} & VAR(2) & 3 & 2 & 2 & 2 & 1 & 1 & 1 & 1 & 1 & 1 & 1 & 1 \\
\hline & BVAR1 & 5 & 5 & 5 & 5 & 5 & 5 & 5 & 5 & 5 & 5 & 5 & 5 \\
\hline & BVAR2 & 4 & 4 & 4 & 4 & 4 & 4 & 4 & 4 & 4 & 4 & 3 & 3 \\
\hline & BVAR3 & 2 & 3 & 3 & 3 & 3 & 3 & 3 & 3 & 3 & 3 & 4 & 4 \\
\hline & BVAR4 & 1 & 1 & 1 & 1 & 2 & 2 & 2 & 2 & 2 & 2 & 2 & 2 \\
\hline \multirow{5}{*}{0.85} & VAR(2) & 3 & 3 & 3 & 3 & 2 & 2 & 1 & 1 & 1 & 1 & 1 & 1 \\
\hline & BVAR1 & 5 & 5 & 5 & 5 & 5 & 5 & 5 & 5 & 5 & 5 & 5 & 5 \\
\hline & BVAR2 & 4 & 4 & 4 & 4 & 4 & 4 & 4 & 4 & 4 & 4 & 3 & 3 \\
\hline & BVAR3 & 2 & 2 & 2 & 2 & 3 & 3 & 3 & 3 & 3 & 3 & 4 & 4 \\
\hline & BVAR4 & 1 & 1 & 1 & 1 & 1 & 1 & 2 & 2 & 2 & 2 & 2 & 2 \\
\hline \multirow{5}{*}{0.9} & VAR(2) & 5 & 5 & 5 & 5 & 4 & 4 & 3 & 3 & 2 & 2 & 1 & 1 \\
\hline & BVAR1 & 4 & 4 & 4 & 4 & 5 & 5 & 5 & 5 & 5 & 5 & 5 & 5 \\
\hline & BVAR2 & 3 & 3 & 3 & 3 & 3 & 3 & 4 & 4 & 4 & 4 & 3 & 3 \\
\hline & BVAR3 & 2 & 2 & 2 & 2 & 2 & 2 & 2 & 2 & 3 & 3 & 4 & 4 \\
\hline & BVAR4 & 1 & 1 & 1 & 1 & 1 & 1 & 1 & 1 & 1 & 1 & 2 & 2 \\
\hline
\end{tabular}


M. O. Adenomon et al.

\section{Continued}

\begin{tabular}{|c|c|c|c|c|c|c|c|c|c|c|c|c|c|}
\hline & VAR(2) & 5 & 5 & 5 & 5 & 5 & 5 & 5 & 5 & 5 & 5 & 2 & 2 \\
\hline & BVAR1 & 4 & 4 & 4 & 4 & 4 & 4 & 4 & 4 & 4 & 4 & 5 & 5 \\
\hline \multirow[t]{5}{*}{0.95} & BVAR2 & 3 & 3 & 3 & 3 & 3 & 3 & 3 & 3 & 3 & 3 & 4 & 4 \\
\hline & BVAR3 & 2 & 2 & 2 & 1 & 2 & 2 & 2 & 2 & 2 & 2 & 3 & 3 \\
\hline & BVAR4 & 1 & 1 & 1 & 2 & 1 & 1 & 1 & 1 & 1 & 1 & 1 & 1 \\
\hline & VAR(2) & 5 & 5 & 5 & 5 & 5 & 5 & 5 & 5 & 5 & 5 & 5 & 5 \\
\hline & BVAR1 & 4 & 4 & 4 & 4 & 1 & 1 & 4 & 4 & 3 & 3 & 4 & 4 \\
\hline \multirow[t]{3}{*}{0.99} & BVAR2 & 3 & 3 & 2 & 2 & 2 & 2 & 3 & 3 & 2 & 2 & 3 & 3 \\
\hline & BVAR3 & 2 & 2 & 1 & 1 & 3 & 3 & 1 & 1 & 1 & 1 & 2 & 2 \\
\hline & BVAR4 & 1 & 1 & 3 & 3 & 4 & 4 & 2 & 2 & 4 & 4 & 1 & 1 \\
\hline
\end{tabular}

\begin{tabular}{|c|c|c|c|c|c|c|c|c|c|}
\hline \multirow{3}{*}{$\begin{array}{c}T=256 \\
\text { AUTOCO } \\
\text { LEVELS }(\delta)\end{array}$} & \multirow{3}{*}{ Models } & \multicolumn{8}{|c|}{ COLLINEARITY $(\rho)$} \\
\hline & & \multicolumn{2}{|c|}{0.85} & \multicolumn{2}{|c|}{0.9} & \multicolumn{2}{|c|}{0.95} & \multicolumn{2}{|c|}{0.99} \\
\hline & & RMSE & MAE & RMSE & MAE & RMSE & MAE & RMSE & MAE \\
\hline \multirow{5}{*}{-0.99} & VAR(2) & 5 & 5 & 5 & 5 & 5 & 5 & 5 & 5 \\
\hline & BVAR1 & 4 & 4 & 4 & 4 & 2 & 3 & 1 & 1 \\
\hline & BVAR2 & 1 & 3 & 2 & 3 & 1 & 1 & 2 & 2 \\
\hline & BVAR3 & 3 & 2 & 1 & 2 & 3 & 2 & 3 & 3 \\
\hline & BVAR4 & 2 & 1 & 3 & 1 & 4 & 4 & 4 & 4 \\
\hline \multirow{5}{*}{-0.95} & VAR(2) & 4 & 2 & 4 & 2 & 5 & 5 & 5 & 5 \\
\hline & BVAR1 & 5 & 5 & 5 & 5 & 4 & 4 & 2 & 2 \\
\hline & BVAR2 & 2 & 3 & 2 & 3 & 1 & 1 & 1 & 1 \\
\hline & BVAR3 & 3 & 4 & 3 & 4 & 3 & 3 & 3 & 3 \\
\hline & BVAR4 & 1 & 1 & 1 & 1 & 2 & 2 & 4 & 4 \\
\hline \multirow{5}{*}{-0.9} & VAR(2) & 1 & 1 & 1 & 1 & 2 & 1 & 5 & 5 \\
\hline & BVAR1 & 5 & 5 & 5 & 5 & 5 & 5 & 3 & 4 \\
\hline & BVAR2 & 3 & 3 & 3 & 3 & 3 & 3 & 1 & 2 \\
\hline & BVAR3 & 4 & 4 & 4 & 4 & 4 & 4 & 4 & 3 \\
\hline & BVAR4 & 2 & 2 & 2 & 2 & 1 & 2 & 2 & 1 \\
\hline \multirow{5}{*}{-0.85} & VAR(2) & 1 & 1 & 1 & 1 & 1 & 1 & 3 & 3 \\
\hline & BVAR1 & 5 & 5 & 5 & 5 & 5 & 5 & 5 & 5 \\
\hline & BVAR2 & 3 & 3 & 3 & 3 & 3 & 3 & 1 & 2 \\
\hline & BVAR3 & 4 & 4 & 4 & 4 & 4 & 4 & 4 & 4 \\
\hline & BVAR4 & 2 & 2 & 2 & 2 & 2 & 2 & 2 & 1 \\
\hline \multirow{5}{*}{-0.8} & VAR(2) & 1 & 1 & 1 & 1 & 1 & 1 & 2 & 1 \\
\hline & BVAR1 & 5 & 5 & 5 & 5 & 5 & 5 & 5 & 5 \\
\hline & BVAR2 & 3 & 3 & 3 & 3 & 3 & 3 & 3 & 3 \\
\hline & BVAR3 & 4 & 4 & 4 & 4 & 4 & 4 & 4 & 4 \\
\hline & BVAR4 & 2 & 2 & 2 & 2 & 2 & 2 & 1 & 2 \\
\hline \multirow{5}{*}{0.8} & VAR(2) & 1 & 1 & 1 & 1 & 1 & 1 & 1 & 1 \\
\hline & BVAR1 & 5 & 5 & 5 & 5 & 5 & 5 & 5 & 5 \\
\hline & BVAR2 & 3 & 3 & 3 & 3 & 3 & 3 & 3 & 3 \\
\hline & BVAR3 & 4 & 4 & 4 & 4 & 4 & 4 & 4 & 4 \\
\hline & BVAR4 & 2 & 2 & 2 & 2 & 2 & 2 & 2 & 2 \\
\hline
\end{tabular}


M. O. Adenomon et al.

\begin{tabular}{|c|c|c|c|c|c|c|c|c|c|}
\hline & VAR(2) & 1 & 1 & 1 & 1 & 1 & 1 & 1 & 1 \\
\hline & BVAR1 & 5 & 5 & 5 & 5 & 5 & 5 & 5 & 5 \\
\hline \multirow[t]{5}{*}{0.85} & BVAR2 & 3 & 3 & 3 & 3 & 3 & 3 & 3 & 3 \\
\hline & BVAR3 & 4 & 4 & 4 & 4 & 4 & 4 & 4 & 4 \\
\hline & BVAR4 & 2 & 2 & 2 & 2 & 2 & 2 & 2 & 2 \\
\hline & VAR(2) & 1 & 1 & 1 & 1 & 1 & 1 & 2 & 2 \\
\hline & BVAR1 & 5 & 5 & 5 & 5 & 5 & 5 & 5 & 5 \\
\hline \multirow[t]{5}{*}{0.9} & BVAR2 & 3 & 3 & 3 & 3 & 4 & 3 & 4 & 4 \\
\hline & BVAR3 & 4 & 4 & 4 & 4 & 3 & 4 & 3 & 3 \\
\hline & BVAR4 & 2 & 2 & 2 & 2 & 2 & 2 & 1 & 1 \\
\hline & VAR(2) & 4 & 3 & 4 & 4 & 5 & 5 & 5 & 5 \\
\hline & BVAR1 & 5 & 5 & 5 & 5 & 4 & 4 & 4 & 4 \\
\hline \multirow[t]{5}{*}{0.95} & BVAR2 & 3 & 4 & 3 & 3 & 3 & 3 & 3 & 3 \\
\hline & BVAR3 & 2 & 2 & 2 & 2 & 2 & 2 & 2 & 2 \\
\hline & BVAR4 & 1 & 1 & 1 & 1 & 1 & 1 & 1 & 1 \\
\hline & VAR(2) & 5 & 5 & 5 & 5 & 5 & 5 & 5 & 5 \\
\hline & BVAR1 & 4 & 4 & 4 & 4 & 4 & 4 & 4 & 4 \\
\hline \multirow[t]{3}{*}{0.99} & BVAR2 & 3 & 2 & 2 & 2 & 3 & 3 & 3 & 3 \\
\hline & BVAR3 & 2 & 3 & 3 & 3 & 2 & 2 & 1 & 1 \\
\hline & BVAR4 & 1 & 1 & 1 & 1 & 1 & 1 & 2 & 2 \\
\hline
\end{tabular}

